\title{
SCIENTIFIC POSTER EXHIBITION
}

\section{Interventional: Brain}

\section{P01:1}

\section{DUAL VOLUME RECONSTRUCTION OF 3D ANGIOGRAPHY IN EVALUATION OF ANEURYSMS TREATED USING ENDOVASCULAR OR SURGICAL APPROACH}

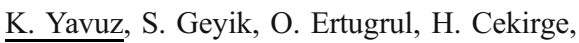
I. Saatci

Hacettepe University, Ankara, Turkey

\section{PURPOSE}

To assess the significance of dual volume reconstruction in $3 \mathrm{D}$ rotational angiography for detecting neck remnant after cerebral aneurysm treatment.

METHODS

The study group included total of 40 cerebral aneurysms following endovascular treatment in 30 patients at sixth month control and surgical clipping in 10 patients within the first week after the surgery which were evaluated with digital subtraction angiography (DSA). 3D rotational angiography was obtained using a flat detector angiographic system (Axiom Artis dBA, Siemens Medical, Erlangen) with 5 seconds acquisition. Postprocessing was performed in Leonardo InSpace workstation (Siemens) with "dual volume reconstruction" technique.

\section{RESULTS}

Three-dimensional angiography provided sufficient visualization of the aneurysm neck in relationship to the parent artery. Seperatecolor visualization of endovascular or surgical material and the contrast-filled vessel enabled accurate information for visualizing the presence of inflow into the aneurysm, if there is any. When present, the extent and the shape of the neck remnant could be better delineated using dual volume reconstruction compared to DSA which enables a better judgement of retreatment indication. CONCLUSION

Dual volume reconstruction in three-dimensional angiographic imaging provides valuable information in the evaluation of the efficacy and stability of endovascular or surgical treatment of cerebral aneurysms. It also helps to assign the follow-up and retreatment protocols when necessary.

\section{PO1:2}

\section{THE USE OF DYNA-CT FOR THE DIAGNOSTIC IMAGING IN NEURORADIOLOGY}

A. Urbanik, T. Turski, T. Popiela, P. Brzegowy

Department of Radiology, Medical College, Jagiellonian University, Kraków, Poland

\section{INTRODUCTION}

The study is evaluating the value of DYNA-CT, a new technique used for diagnosing tumors and vascular pathologies of central nervous system and in the interventional neuroradiology.

Material and Method:

80 examinations were performed with the DYNA-CT technique using Axiom Artis (Siemens) with flat detector system and 3D rotational angiography (in 62 cases complementary diagnostic examination and in the remaining 18 as a control after or during neuroradiological interventional procedures). DYNA-CT was performed without and with intraarterial contrast administration. DynaCT provided images obtained during the rotational angiography similar to those provided by $\mathrm{CT}$ scans.

RESULTS

In case of well-vascularized tumors, DYNA-CT allows to evaluate the localization of the pathologies in relation to intracranial bone structures and topographic lines for planning neurosurgical procedures. Brain examination with DYNA-CT during and after embolization of cerebral aneurysm and the lack of visualized bleeding from the aneurysmal sac eliminated the need for patient's transportation to the CT lab for the control examination.

CONCLUSIONS

DYNA-CT is supplementing DSA angiography by allowing spatial localization of pathologies in relation to cranial bone structures, which is especially useful for planning neurosurgical procedures. Additionally, DYNA-CT may be used instead of standard CT of head as a control examination to detect complications of interventional neuroradiology procedures.

\section{P01:3}

ENDOVASCULAR TREATMENT OF INTRACRANIAL FUSIFORM ANEURYSM USING 'BALLOON-IN-STENT' TECHNIQUE

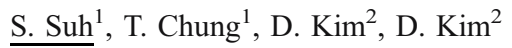

${ }^{1}$ Yongdong Severance Hospital, Yonsei University, Seoul, South Korea; ${ }^{2}$ Yonsei University, College of Medicine, Seoul, South Korea

\section{PURPOSE}

Fusiform aneurysms, which incorporate the branch vessel or are not a candidate for destructive treatment, are a unique therapeutic challenge. This study is to evaluate the endovascular treatment of intracranial fusiform aneurysm using 'balloon-in-stent' technique. METHODS

From September 2005 to March 2007, 10 patients (M:F=3:7, mean age $=62.3$ years) with intracranial fusiform aneurysm were 
treated with 'balloon-in-stent' technique in combination with endovascular coiling. Two patients were performed for internal carotid aneurysm and eight for vertebrobasilar trunk aneurysm. In the first step of 'balloon-in-stent' technique, a stent was placed across the neck of the aneurysm to obtain parent vessel reconstruction. The second step was performed sequentially, which coil embolization was performed with a compliant occlusion balloon in place within stent to protect the stented parent vessel lumen. This process was repeated until satisfactory aneurysm embolization was achieved. The extent of coil packing was categorized as three grades using control angiography after embolization: complete, neck remnant, loose packing.

Angiographic follow-up was performed 6 months after embolization for evaluation of stent patency and coil packing.

RESULTS

Endovascular treatment with 'balloon-in-stent' technique was technically successful in all patients. There was no complication related to the procedure and complete occlusion was obtained in 6 patients, neck remant in 3 patients and loose occlusion in 1 patients. All patients were recovered well. Follow-up angiograpy after 6 month showed patency of parent artery without aneurysm recanalization. Conclusion:

The balloon-in-stent technique may provide a feasible and safe treatment strategy for the management of intracranial fusiform aneurysms that are not amenable to deconstructive embolization.

\section{PO1:4}

CEREBRAL SINOVENOUS THROMBOSIS : IMAGING DIAGNOSIS AND MANAGEMENT

\section{Shy ${ }^{1}$, C. Chen ${ }^{2}$, W. Chen ${ }^{2}$, H. Hung ${ }^{2}$}

${ }^{1}$ Ping-Tung Christian Hospital, Ping-tung, Taiwan;

${ }^{2}$ Taichung Veterans General Hospital, Taichung, Taiwan

\section{PURPOSE}

Cerebral sinovenous thrombosis (CSVT) is an uncommon disorder that affects the dural venous sinus and cerebral vein. In this study, we present the imaging findings of CSVT in CT, MRI, MR venography (MRV) and the digital substraction angiography (DSA) respectively, in correspondence to the clinical appearance and the outcome of management.

\section{MATERIAL AND METHODS}

Thirty-four patients, aged from 2 weeks to 54 years old, with clinical manifestations from headache to a progressive neurological deficit and conscious loss, were enrolled in this study. The patients received imaging study including $\mathrm{CT}$ in 28, MRI in 24, 2D TOF and contrast-enhanced MRV in 19, DSA in 18 cases. Sixteen patients received systemic intravenous heparinization and twelve patients received endovascular thrombolytic treatment with urokinase combining with anticoagulant therapy.

\section{RESULTS}

The venous sinus and cerebral vein involved appearaed abnormal imaging findings. The abnormal findings were detected in 16 cases on non-contrast enhanced CT and in 10 cases on contrastenhanced CT. On MRI, CSVT was staged as acute stage (7 cases), subacute stage (12 cases) and chronic stage ( 5 cases). Absence or incomplete filling of dural sinuses was noted in 17 cases on MRV. DSA demonstrated dynamic changes in 16 cases. The abnormal parenchymal change on CT and/or MR findings were 6 cases in stage I, 5 cases in stage II, 8 cases in stage III, 10 cases in stage IV and 5 cases in stage V. Following CSVT, the neurological deficits were resolved in 21 cases, while partial remaining in 10 cases. Death was inevitable in 3 cases. Recurrence occurred in 3 cases. CONCLUSION

Clinical suspicion and excellent neuroimaging are crucial in making diagnosis of CSVT. Proper management with anticoagulant and/or endovascular thrombolytic therapy is mandatory in preventing propagation of thrombosis and improving the clinical outcome.

\section{P01:5}

\section{CONTRAST AGENT EXTRAVASATE AFTER INTRAARTERIAL RECANALIZATION IN THE ANTERIOR CIRCULATION, A RISK FACTOR FOR BLEEDING?}

A. Ragoschke-Schumm, I. Mader, M. Schumacher, A. Berlis

Department of Neuroradiology, Freiburg, Germany

\section{PURPOSE}

Bleeding complications are frequently observed in patients with intraarterial recanalization in ischemic stroke. They can cause an impaired clinical outcome or death of the patient. Early contrast agent extravasates (ECAE) are frequently observed in patients with intraarterial recanalization, this is regarded as a disturbed bloodbrain barrier. The aim of this study is to assess if ECAE predict severe bleeding complications. As there are different clinical courses and therapeutic windows for vascular occlusion in the anterior and the posterior circulation we assessed only patients with recanalization in the anterior circulation for this study.

Methods: Retrospective study. From 2003 to 2006151 intraarterial recanalization therapies were performed at our department in Freiburg. Patients were treated either with i.a.-rtPA, by combination of rtPA and mechanic recanalization or mechanic recanalization alone. All patients were assessed for appearance of ECAE, bleeding complications and clinical outcome at time of discharge. At least one postinterventional imaging by $\mathrm{CT}$ or MRI was performed. Bleedings were divided in hemorrhagic transformation, small bleeding which did not exceed $30 \%$ of the resulting area of infarction and large bleedings with bad clinical outcome as assessed by the modified Rankin Scale (mRS).

\section{RESULTS}

$110(73 \%)$ of the included 151 patients showed ECAE, mostly in the ipsilateral basal ganglia or insula cortex. 34 (23\%) Patients developed hemorrhagic transformations without clinical impairment, $15(10 \%)$ showed small bleedings with no or temporary clinical impairment and 18 (12\%) Patients suffered from large bleeding complications with severe symptoms at discharge (mRS 4 to 6$)$. Of these 18 patients 16 (14\% of patients with extravasate) 
showed early contrast agent extravasation while 2 (5\% of patients without extravasate) did not.

\section{CONCLUSIONS}

Early contrast agent extravasates occur very frequently (73\%) in patients with intraarterial recanalization. Patients with this condition tend to suffer more often from severe bleeding complications (14\% vs. $5 \%$ ). In patients with extravasates arterial blood pressure and coagulatory status should be carefully monitored and blood pressure peaks must be avoided. Further evaluation and studies are needed to show if there is an increased bleeding risk for early secondary prevention with platelet inhibitors.

\section{PO1:6}

\section{EXTRACRANIAL INTERNAL CAROTID STENTING WITHOUT DISTAL PROTECTION DEVICES}

\author{
E. Perez Cuenca, F. Bravo Rodríguez, R. Oteros Fernández, \\ F. Delgado Acosta \\ Hu Reina Sofia Córdoba Spain
}

\section{PURPOSE}

The aim of this study is to show our experience in carotid stenting of internal carotid artery without cerebral protection devices, the most of them doing the dilation after stent placement. And to value the number of cerebral embolism accidents that take place. MATERIALS AND METHODS

We review 100 patients, in 8 of them we could not place the stent due to several causes, so we evaluated 92 patients with 96 carotid stenosis which were treated in our institution. 17 of the 96 procedures were made in women. The average age was 65 years (aged between 41 and 84 years). $96 \%$ had a greater stenosis of $70 \%$. In 59 cases angyoplasty was done after stent placement. Procedure was performed without protection device in all patients. We analyzed neurological complications by means of basic neurological exploration in Neuroradiology Room and in the 24 following hours. Also we gathered hemodynamic alterations.

\section{RESULTS}

In $97 \%$ stent was implanted with smaller residual stenosis of $30 \%$. Only 2 patients has neurological complications: an embolic infarct secundary to internal carotid dissection post-angioplasty and an hematoma by reperfusion. The rest of complications were: $20,2 \%$ vagal reactions ( 9 of them didn't need treatment with atrophyna), local complications secondary to femoral punción $(3 \%)$ and others $(3 \%)$.

\section{CONCLUSIONS}

In our serie, the stent placement for the treatment of symptomatic carotid stenosis without cerebral protection devices seems safe. The rate of neurologycal complications was low, only $2 \%$. The stent could act like a protection system and it would avoid the excessive manipulation and that the procedure becomes too long. The vagal reactions with bradycardia were the most frecuent complication and in the half of the cases didn't need treatment.

\section{P01:7}

EFFECTIVE PATIENT-HEAD FIXATION
FOR THE NEURO-INTERVENTIONAL PROCEDURE
UNDER LOCAL ANESTHESIA: USEFULNESS
OF OUR NEW CUSTOM-MADE HEAD-FIXING
SYSTEM (ZORRO-MASK SYSTEM)

M. Nagahata ${ }^{1}$, Y. Abe ${ }^{1}$, S. Ono ${ }^{1}$, M. Aoki ${ }^{1}$, H. Miura $^{1}$, T. Ohata ${ }^{1}$, H. Kondo ${ }^{1}$, Y. Hatayama ${ }^{1}$, F. Tsushima ${ }^{1}$, H. Kawaguchi ${ }^{1}$, K. Morimoto ${ }^{1}$, H. Seino ${ }^{1}$, K. Basaki ${ }^{1}$, N. Shimamura ${ }^{2}$, H. Ohkuma ${ }^{2}$

${ }^{1}$ Department of Radiology, Hirosaki University School of Medicine, Hirosaki, Japan; ${ }^{2}$ Department of Neurosurgery, Hirosaki University School of Medicine, Hirosaki, Japan

\section{BACKGROUND \& PURPOSE}

Patient-head fixation, especially in cases with disturbed consciousness, is an important factor to perform a safer neuro-intervention under local anesthesia. For the neuro-interventional procedure, we modified a patient immobilization system used in radiotherapy. We report the usefulness of our new head-fixing system for neurointervention.

METHODS

A thermo-formable plastic board (Thermoshell, Alcare Inc. Tokyo, Japan) and a moldable pillow (Moldcare, Alcare Inc. Tokyo, Japan) are generally used during radiotherapy for head and neck tumors in Japan. From May 2006, we have performed endovascular neurosurgery with a custom-made face mask covering the upper half of the face using the Thermoshell and also a custommade pillow (Moldcare) fitted to each patient. We call our new head-fixing system a Zorro-mask System. It takes only about 20 minutes to make a Zorro-mask system for each patient.

\section{RESULTS}

Our Zorro-mask system did not influence the imaging quality of the fluorography or DSA. Skin dose of radiation exposure under the mask increased only $1 \%$ on fluorography and $2 \%$ on DSA in average. Among the patients with disturbed consciousness, frequency of head motion disturbing the fluoroscopic catheterization was significantly lower in the cases with Zorro-mask System than in the cases without Zorro-mask system. Unconsciously head motion was well controlled. No patient complained of any discomfort from the Zorro-mask system. There has not been any harmful event due to the Zorro-mask system.

\section{CONCLUSION}

Our Zorro-mask system is a simple and useful head-fixing device for immobilization during neuro-interventional procedures under local anesthesia.

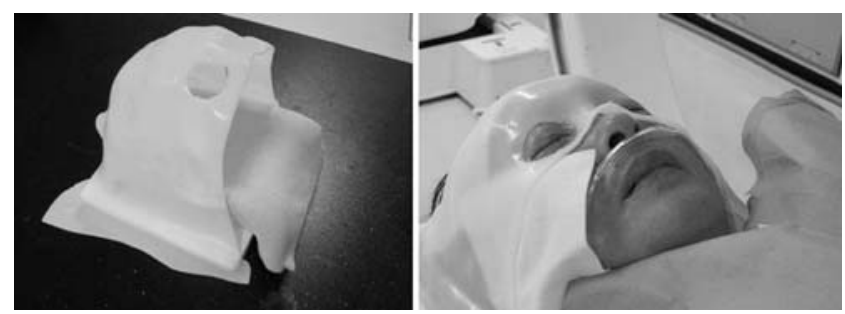




\section{PO1:8}

OUR EXPERIENCE IN NEUROFORM STENT-ASSISTED COIL EMBOLISATION OF WIDE-NECKED INTRACRANIAL ANEURYSMS (A PROPOS 5 CASES)

S. Milosevic Medenica ${ }^{1}$, V. Branca ${ }^{2}$, B. Prstojevic ${ }^{1}$, V. Vukovic ${ }^{1}$ ${ }^{1}$ Clinical Center of Serbia, Belgrade, Serbia,; ${ }^{2}$ Ospedale Maggiore, Milano, Italy,

\section{PURPOSE}

The aim of this study was to evaluate the efficiency of coil embolisation of wide necked aneurysms using stent-assisted technique.

PATIENTS AND METHODS

Since January 2007. 5 patients with wide-necked intracranial aneurysms underwent stent-assisted endovascular interventions. Patients ranged in age from 46 to 63 years and were all women. There were 4 aneurysms on the internal carotid artery (2 on the $\mathrm{C} 2 \mathrm{C} 1$ segment and 2 on the $\mathrm{C} 5 \mathrm{C} 4$ segment) and 1 aneurysm on the P1 segment of the posterior cerebral artery. All aneurysms were non-bleeding, with long standing clinical symptoms. During the endovascular procedures we used Neuroform stents (Boston scientific), while the occlusion of the aneurysms was performed by microcoils Matrix and GDC (Boston Scientific). We have had no difficulties in the delivery of stents. Premedication was performed with low-molecular anticoagulant (Fraxarin) and standard Heparin during the intervention, while postprocedural antithrombotic medication was prescribed for all patients during 6 weeks.

RESULTS

Complete occlusion of the aneurysms was achieved in all cases. We did not have any periprocedural complications. Since now there was no postoperative mortality, neither morbidity. Control angiography has not yet been done and it is planned for 6 months after the procedure.

CONCLUSION

According to preliminary results, intracranial use of self-expandible stents during the treatment of wide-necked aneurysms has shown as efficient and safe method. For more reliable results we need larger experience and long term follow up.

\section{P01:9}

\section{ENDOVASCULAR TREATMENT OF FUSIFORM ANEURYSM} OF BASILAR ARTERY ASSOCIATED WITH GLYCOGENOSIS TYPE 2 (POMPE DISEASE)

G. Lodoli $^{1}$, R. Liserre ${ }^{1}$, C. Ambrosi ${ }^{1}$, E. Boccardi ${ }^{2}$, M. Pavia ${ }^{1}$, R. Gasparotti ${ }^{1}$

${ }^{1}$ Neuroradiology Unit, Ospedali Civili, Brescia, Italy;

${ }^{2}$ Interventional Neuroradiology Unit, Ospedale Niguarda, Milano, Italy

\section{PURPOSE}

To report a case of a glycogenosis type 2 with associated fusiform vertebrobasilar aneurysm.

\section{CASE REPORT}

A 28-year-old woman presented with a left side hemiparesis associated with dysarthria and right ptosis. Emergency CT scan showed a brainstem hematoma extending from the right cerebral peduncle to the right middle cerebellar peduncle; CT-angiography and digital subtraction angiography (DSA) demonstrated a giant fusiform aneurysm of vertebrobasilar junction with ectatic basilar and vertebral arteries and mass effect on brainstem. The patient was affected by glycogenosis 2, a rare disease etiologically characterized by a deficiency of á-glycosidase and clinically characterized by cardiovascular diseases and neurological symptoms. To our knowledge, only one case of giant intracranial aneurysm associated with glycogenosis type 2 has been reported, probably caused by pathological glycogen deposit in the vessel walls. After 10 months of rehabilitative therapy, the patient developed a brainstem ischemia due to acute partial thrombosis of the aneurysm, which was demonstrated by a magnetic resonance (MR) examination. After discussion of the therapeutic options with neurosurgeons we opted for the endovascular treatment with stent-assisted coiling of the aneurysm.

RESULTS

Endovascular treatment was iniziated with placement of a selfexpanding stent in the basilar artery with the distal end at the top of basilar artery and the proximal end in the left vertebral artery distal to postero-inferior cerebellar artery (PICA). A second stent of the same size and length was positioned inside the first one. Finally the right vertebral artery was occluded with Guglielmi detachable coils (GDC) distal to the PICA origin. Subsequent MR scan demonstrated decrease of brainstem compression and oedema; MR angiography confirmed right vertebral artery occlusion and patent flow in both PICAs, basilar artery, superior cerebellar arteries and posterior cerebral arteries.

CONCLUSION

Overlapping stent placement may be a viable option in dissecting or fusiform intracranial aneurysms that are not amenable to open surgical treatment or endovascular coil embolization.

\section{P01:10}

\section{DEVELOPMENTAL VENOUS ANGIOMAS COMPLICATED BY HEMORRHAGE IN DIFFERENT PRESENTATIONS}

\author{
Y. Kiroglu $^{1}$, I. Oran ${ }^{2}$, A. Sirikci ${ }^{3}$ \\ ${ }^{1}$ Pamukkale University School of Medicine, Denizli, Turkey; ${ }^{2}$ Ege \\ University School of Medicine, Izmir, Turkey; ${ }^{3}$ Gaziantep \\ University School of Medicine, Gaziantep, Turkey
}

\section{PURPOSE}

We evaluate 8 hemorrhagic developmental venous angiomas (DVA) in rare presentations 4 arterialized developmental venous angiomas 
(ADVA), 2 DVA associated with cavernous malformation, 1 DVA which serves as draining vein of a true AVM and 1 DVA with drainage vein thrombosis .

Material and Method:

The study population was obtained from the patients who were diagnosed intracranial hemorrhagic DVA. There were total of 8 patients ( 4 males and 4 females). CT or/and MR and conventional angiographic examinations were performed at the initial symptoms in all patients. The patients were divided into four groups to a better radiological analysis; (I) ADVA, (II) DVA associated with cavernous angioma (CA), (III) DVA with AVM, (IV) DVA with trombosed drainage vein.

RESULTS

In four patients of group (I); intraparanchymal hemorrhage were detected in MR images. Classic DVA appearance was exist in two while it was suspicious in the other two patients on MRI. Angiographic images showed capillary blush and early venous filling in arterial phase in all patients diagnosed as ADVA.

In two patients of group (II); cavernous angiomas were diagnosed on MRI. DVA diagnosed in typical images in angiographic examination in both patients and DVAs were evidently within the hemorrhagic lesion areas.

In one patient of group (III); hemorrhage and suspicious AVM diagnosed on MRI. On angiographic evaluation, an AVM with a small nidus and an adjacent DVA were diagnosed. Collecting veins of DVA in caput medusa formation, which converged, with draining vein of AVM was detected. One patient of group (IV) was diagnosed a DVA which has a trombosed draining vein.

\section{CONCLUSION}

We evaluated the rare and different hemorrhagic presentations of DVAs which illustrates its the potential complexity. We recommend that MR and cerebral angiography should be used as complementary methods and evaluated carefully in especially suspicious hemorrhages and neurological symptoms with DVAs.

\section{P01:11}

\section{CLINICAL APPLICATION OF 3D ROADMAP IN NEUROINTERVENTION}

\section{J.H. Kim, Y. Partk, H. Han}

Konayang University Hospital, Daejeon, South Korea

\section{PURPOSE}

Three dimensional roadmap (3D roadmap) is a novel technique combining rotational angiography and 2D fluoroscopy. We applied this technique to neurovascular intervention to investigate clinical feasibility and usefulness.

\section{MATERIALS AND METHODS}

Twenty two patients were enrolled, including coil embolization of cerebral artery aneurysm (13/22), embolization of dural AVF (2/ $22)$, tumor embolization $(5 / 22)$, and embolization of bleeding vessels $(2 / 22 ; 1$ iatrogenic, 1 traumatic). All procedures were performed by two neurointerventionalists (1 radiologist, 1 neurosurgeon) with clinical experiences using conventional roadmap system. 3D roadmap was used as an imaging tool for microcatheter advancement and delivery of embolic materials including coils, particles, and NBCA in all cases. We evaluated feasibility, usefulness, and limitation of $3 \mathrm{D}$ roadmap as compared with conventional roadmap.

\section{RESULTS}

Creating 3D roadmap was possible with one time contrast media injection in all patients. It was maintained until finishing the procedures in 19 patients. Re-trial was needed in 3 patients due to unwanted patient's motion or operator's error. Easy navigation of microcatheter to target vessels or lesions was experienced in all patients. In embolotherapy of dural AVF, tumor, and bleeding, the operators felt more convenient as compared with when they had performed procedures using conventional roadmap system. Easy perception of vascular branching anatomy in ECA system was possible and there was no need for re-trial of roadmap making to select vessels. In aneurysm coiling, there were no distinguished advantages as compared with conventional roadmap system. Instead, exact catching 3-D configuration of deployed coils was difficult.

CONCLUSION

$3 \mathrm{D}$ roadmap is clinically feasible in neurovascular intervention. It permits precise and swift approach to the target vessels or lesions in the patients with uncontrolled fine movement disrupting roadmap image or complex vascular anatomy. However, definite advantages were not noted in aneurysm coiling as compared with conventional roadmap.

\section{P01:12}

RADIOSURGERY FOR CEREBRAL ARTERIO-VENOUS MALFORMATIONS: A STUDY OF PROGNOSTIC FACTORS OF CURE AND PREDICTIVE FACTORS OF OCCURRENCE OF RADIONECROSIS IN 179 PATIENTS

$\underline{\text { N. Blanchard }}^{1}$, R. Anxionnat ${ }^{2}$, V. Bernier ${ }^{1}$, D. Peiffert ${ }^{1}$, I. Buchheit ${ }^{1}$, S. Bracard ${ }^{2}$, L. Picard ${ }^{2}$

${ }^{1}$ Department of Radiotherapy. Centre Alexis Vautrin Cancer Center, Vandoeuvre-Les-Nancy, France; ${ }^{2}$ Department of Diagnostic and Therapeutic Neuroradiology. University Hospital, Nancy, France

\section{PURPOSE}

To evaluate the prognostic factors of brain artério-venous malformations (BAVMs) obliterations and the predictive factors of occurrence of radionecrosis in 179 patients treated by radiosurgery between 1992 et 2004 in Nancy.

MATERIALS AND METHODS

179 patients with 179 BAVMs, whom 90\% were previously embolized, were treated by radiosurgery with a LINAC using arctherapy in $84 \%$ and multileaf collimators (MLCs) with fixed fields 
in $16 \%$. The mean follow-up was 42 months (6-131). MRI was performed at 6 months and when radionecrosis was clinically suspected. First angiography was performed at 2 years. Predictive factors of angiographic cure and of occurrence of radionecrosis were studied by a multivariate statistical analysis.

\section{RESULTS}

The mean peripheral and maximal dose was respectively 17.7 Gy and $24.2 \mathrm{~Gy}$. The mean volume of the target was $8.8 \mathrm{~cm} 3$. Cure was obtained in $82.7 \%$ with a mean time of 3.1 years. Good prognostic factors were: a regular shape of the target $(R R=2.8, p<0.0001)$, less than 4 embolizations $(\mathrm{RR}=2.9, \mathrm{p}<0.0001)$, peripheral dose superior to 18 Gy $(R R=2, p=0.0002)$, target volume inferior to $8 \mathrm{~cm} 3(\mathrm{RR}=1.9, \mathrm{p}=0.0002)$. Radionecrosis occurred in $11.2 \%$ with a mean time of 18 months (range, 4-60) and persistent sequellae in $25 \%$. Unfavourable predictive factors were: fixed fields and multileaf collimators (MLC3D Line RR $=9.1, p<0.0001$ and BRAINSCAN $\left.{ }^{\circledR} R R=15.1, p=0,01\right)$ versus arc-therapy, age less than $30(R R=2.5, p=0,04)$, depth of the target superior to $7 \mathrm{~cm}$ $(\mathrm{RR}=7.6, \mathrm{p}=0,008)$, and more than $11 \mathrm{~cm} 3$ of brain tissue volume of parenchyma receiving $12 \mathrm{~Gy}, \mathrm{~V} 12 \mathrm{~Gy}(\mathrm{RR}=7.8, \mathrm{p}=0,05)$.

\section{CONCLUSION}

This study confirms that the peripheral dose must be superior to 18 Gy for efficacy of radiosurgery with LINAC and that it seems important to reduce V12 Gy to decrease risk of radionecrosis.

\section{P01:13}

\section{ENDOVASCULAR TREATMENT OF DURAL ARTERIOVENOUS FISTULA OF THE CAVERNOUS SINUS. ANATOMIC STUDY AND CORRELATIONS WITH TECHNICAL CHOICES}

C. Macedo De Freitas, S. Bracard, R. Anxionnat, A. Lebedinsky, N. Pinheiro JR, G. Andrade, D. Iancu, L. Picard

${ }^{1}$ Department of Diagnostic And Therapeutic Neuroradiology.

University Hospital, Nancy, France

\section{PURPOSE}

To demonstrate the importance of the radioanatomy of the dural arteriovenous cavernous fistula (DAVCFs) for the choice of the technique of endovascular treatment.

\section{PATIENTS AND METHOD}

Fourteen patients with DAVCF were reviewed. Clinical signs were proptosis $(10 / 14)$, chemosis $(10 / 14)$, VIth nerve palsy $(8 / 14)$, retro ocular pain (4/14) and visual loss (2/14).

The fistula was most often located at the posterior part of the cavernous sinus (12/14). Arterial feeders were always multiple: intracavernous branches of the carotid siphon, pharyngeal arteries, middle meningeal arteries and foramen rotundum arteries. Venous drainage was the superior ophthalmic vein exclusively in $7 / 14$, both superior ophthalmic vein and inferior petrous sinus in $4 / 14$, cortical veins exclusively in $2 / 14$ and no venous drainage was seen in one case.
Venous approach was attempted in $12 / 14$ and failed in $2 / 14$. In successful cases, the fistula was reached by superior ophthalmic vein in 6 cases and by inferior petrous sinus in 4 cases. The fistula was then selectively occluded using coils and we always tried to preserve the permeability of the cavernous sinus. Anatomical and technical points concerning these 2 approaches are detailed.

\section{CONCLUSION}

Selective occlusion of the fistula by venous approach is the treatment of choice of DAVCFs. In our experience, the ophthalmic superior vein via the facial vein, when patent, allowed us quite easily to reach both anterior but also posterior part of the cavernous sinus.

\section{P01:14}

\section{PREOPERATIVE EMBOLIZATION OF INTERNAL CAROTID ARTERY BRANCHES AND PIAL VESSELS IN HYPERVASCULAR BRAIN TUMORS}

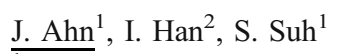

${ }^{1}$ Yonsei University College of Medicine, Seoul, South Korea;

${ }^{2}$ College Of Medicine, Pochon Cha University, Seongnam, South Korea

\section{INTRODUCTION}

Preoperative embolization is an effective method used to reduce intraoperative bleeding and operative difficulty in hypervascular brain tumor surgery. However, embolization of the internal carotid artery (ICA) and pial tumor feeding branches has certain limitations.

Methods: From March 2000 to November 2006, 7 patients underwent superselective tumor embolization for hypervascular brain tumor. Six tumors were extra-axial (5 meningiomas, 1 solitary fibrous tumor) and 1 was intra-axial (metastatic hepatocellular carcinoma).

RESULTS

In all cases, feeding vessels from ICA branches or pial arteries were successfully embolized using superselective microcatheterization. A provocative test was applied in 3 cases that had tumors adjacent to the motor cortex. Angiographic devascularization was slight to extensive. Mean devascularization on postembolization MRI ranged from 40 to $80 \%$ (mean, $62.9 \%$ ). Two patients $(28.6 \%$ ) suffered embolization-related complications (1 loss of retinal brush, 1 postembolization seizure).

\section{CONCLUSION}

Superselective embolization of ICA branches or pial vessels should be performed if the several conditions are met, especially angiographic findings, preexisting neurologic deficits, provocative test, and technical feasibility. If the ICA embolization for hypervascular tumor is successfully achieved, the bleeding loss and operative risk can be reduced. 


\begin{tabular}{|c|c|c|c|c|c|c|c|}
\hline $\mathrm{Cas}$ & $\begin{array}{l}\text { Syon } \\
\text { Se }\end{array}$ & Citad pocstes & Taner kain & $\begin{array}{l}\operatorname{lax} \\
\min \left(\mathbf{a x}^{\prime}\right)\end{array}$ & Feley wesd & $\begin{array}{l}\text { Neplask } \\
\text { mangin }\end{array}$ & tangates \\
\hline 1 & $9 x$ & 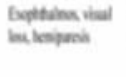 & $\begin{array}{l}\text { Ohe anciur } \\
\text { nidt andi ina }\end{array}$ & $6 \times 5 \times 4$ & $\begin{array}{l}\text { sta bes ots } \\
\text { seiviont } \\
\text { mi }\end{array}$ & Yo & Selep frow inar \\
\hline 2 & si & Meppresh kites & Conont & $9 \times 9 \times 6$ & $\operatorname{sucs}$ & so & Mol noingin \\
\hline 3 & an & Antipued & fath & $5 \times 905$ & $\operatorname{sen} 04$ & so & Apinews maipipe \\
\hline 4 & sin & Med desp & $\begin{array}{l}\text { Me hrediting } \\
\text { Oapted (at) }\end{array}$ & $\begin{array}{l}3 \times 2 \times 4 \\
4 \times 6 \times 5\end{array}$ & $\begin{array}{l}m \\
\text { ots }\end{array}$ & $\infty$ & Mpteckly arines \\
\hline 9 & $\operatorname{men}$ & Vial las deneth & Inenteal & $9 \times 6 \times 6$ & $\operatorname{sen}$ asen & N & Mnitphtimaw seingima \\
\hline 1 & कN & Maripenis & Connt & $6 \times 7=5$ & mucu & $N$ & 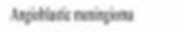 \\
\hline 1 & si & Crail ane pily & Straxchensu ins & $6 \times 35$ & sen & so & 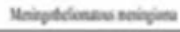 \\
\hline
\end{tabular}

\begin{tabular}{|c|c|c|c|c|c|c|c|}
\hline $\mathrm{Cos}$ & $\begin{array}{l}\text { Intulnd } \\
\text { ica } \\
\text { imole }\end{array}$ & fabled anerith & 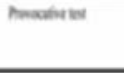 & Moviogtis & $\begin{array}{l}\text { Meidunatirusie } \\
\text { N }\end{array}$ & Cinplowes & 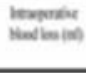 \\
\hline 1 & ase & 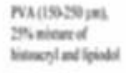 & $\mathbf{M}$ & edanit & $n$ & Imad itid & 130 \\
\hline 2 & $\mathrm{cu}$ & 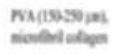 & Andine appate & molest & s & $\mathrm{N}=$ & 20 \\
\hline 3 & ost & 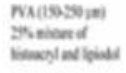 & nipoulve & edimint & $\Rightarrow$ & Sivn & 6 \\
\hline 4 & mota & $\begin{array}{l}\text { walassini } \\
\text { nomelefipn }\end{array}$ & $\mathrm{M}$ & water & in & $\mathrm{Sen}$ & 10 \\
\hline 9 & ans & $\begin{array}{l}\text { Wuisosins } \\
\text { eb }\end{array}$ & $\mathbf{M}$ & moder & " & $\mathrm{N}=$ & 60 \\
\hline 6 & $\mathrm{ca}$ & 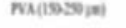 & And us apest & eamole & 7 & $\mathrm{Nen}$ & so \\
\hline 1 & ses & nactosejai & $\mathbf{M}$ & enomint & $n$ & $\mathrm{Kan}$ & 39 \\
\hline
\end{tabular}

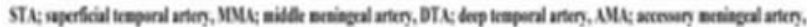

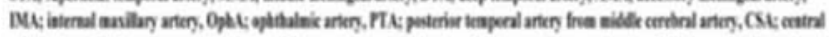

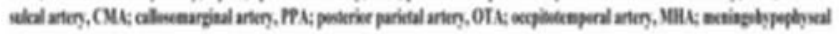
anten,

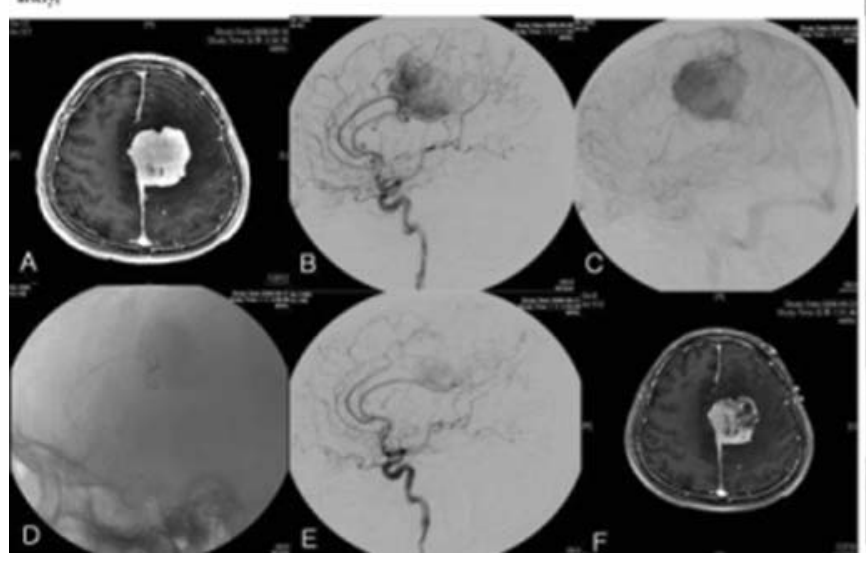

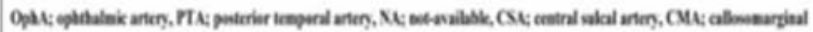

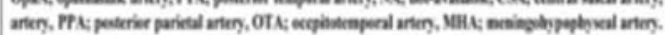

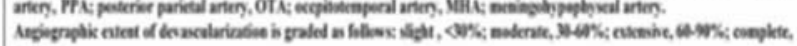
Anowing

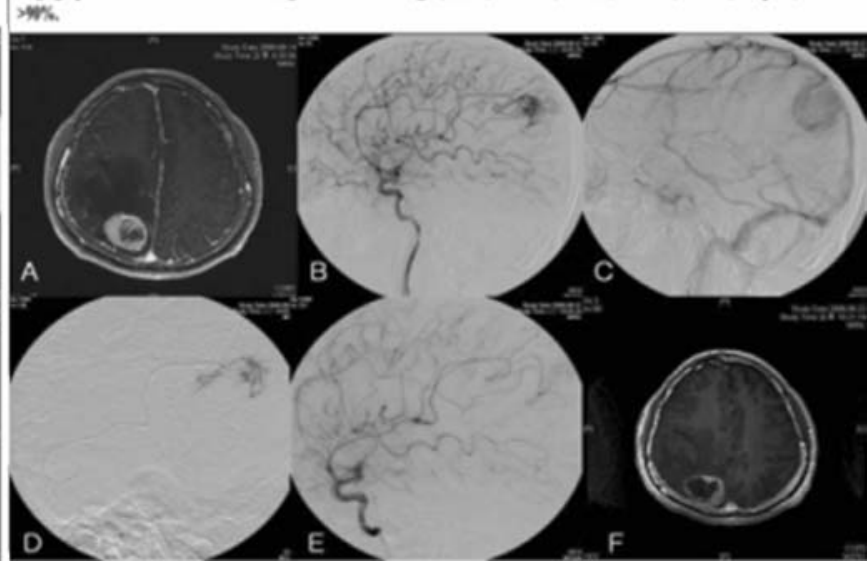

\section{Interventional : Spine}

\section{PO2:1}

\section{PERCUTANEOUS FEMOROPLASTY UNDER CT FLUOROSCOPIC GUIDANCE IN THE TREATMENT OF PROXIMAL OSTEOLYTIC METASTASES AND ADVANCED MULTIPLE MYELOMA.}

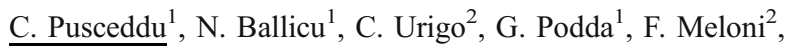
G. Meloni ${ }^{2}$

${ }^{1}$ Oncology Hospital Businco, Cagliari, Italy; ${ }^{2}$ Radiology Department of University, Sassari, Italy

\section{PURPOSE}

To evaluate the feasibility and safety under computer thomography $(\mathrm{CT})$ and fluoroscopically guided for reparing the extensive osteolytic defects of the proximal femur with prophylactic bonecement injection in patients with refractory pain and high risk of fracture.

\section{METHOD AND MATERIALS}

Three women (mean age 62 years) affected by metastatic breast carcinoma (two cases) and advanced multiple mieloma (a case) with large osteolytic area of head and nech femur at high risk of fracture were treated with bone-cement injection. The patients were unsuitable candidates for surgery according to multidiscplinary consensus.
The lesions were accessed using the 10-gauge bone biopsy needle under CT fluoroscopic guidance. In the patients with metastatic carcinoma, radiofrequency ablation (RFA) had been previously performed to destroy tumerous tissue. Subsequnty, under fluoroscopic guidance $8 \mathrm{~mL}(5-12 \mathrm{~mL})$ of polymethylmetacrylate (PMMA) was injected into the lesions.

RESULTS

Thecnical success was achieved and no methylmetacrylate leakage detected. Pain relief was complete within 1 week in all cases. No broke femur's complication occurred after two months follow-up. CONCLUSION

Percutaneous femoroplasty under CT fluoroscopic guidance is feasible and safe in the osteolityc head and nech femoral metastases and multiple mieloma to obtain pain relief and to prevent patological fractures.

\section{PO2:2}

PERCUTANEOUS VERTEBROPLASTY

WITH OR WITHOUT RADIOFREQUENCY (RF) ABLATION OF THE OSTEOLITYC VERTEBRAL METASTASES.

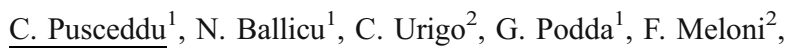
G. Meloni ${ }^{2}$

${ }^{1}$ Oncology Hospital Businco, Cagliari, Italy; ${ }^{2}$ Sassari University, Sassari, Italy 


\section{PURPOSE}

To report our findings on the technical feasibility, local efficiency, incidence of complications and outcome of vertebroplasty with or without RFablation in the treatment of painful vertebral metastases in patients considered inelegible for surgery.

\section{METHOD AND MATERIALS}

From January 2005 to November 2006, 21 consecutive patients were treated for a total of 24 vertebroplasties. In 5 cases, RF ablation has been previously performed to destroy tumerous tissue and to facilitate optimal methylmethacrilate (PMMA) spreading. All patients underwent evaluation with MRI and CT prior to commencing therapy. Inclusion criterion was painful vertebral collapse, whereas exclusion criteria were epidural infiltrations but not infiltrations and the erosion of the posterior wall, seen in 7 cases. An immediate consequence was an asymptomatic leak of the acrylic cement in the vertebral canal at a lumbar level, seen in 3 cases with interruption of the posterior wall.

RESULTS

Technical success was achieved in all patients with a reduction or disappearance of pain within one week of therapy (VAS scores ranged from 7.6 to 1.6). Osteoplasty was facilitated by the previous RF ablation in those patients who underwent combined therapy. Six of 21 patients died as a result of illness progression. Pain relief in 15 patients during follow-up, 4-12 months (8 months average)was good.

\section{CONCLUSION}

Today, percutaneous vertebroplasty with or without RF ablation, represents an important palliative therapy for patients affected by metastatic vertebral collapse. Posterior somatic wall interruption is not an absolute controindication to therapy.

\section{PO2:3}

\section{PERCUTANEOUS IMAGE-GUIDED FEMOROPLASTY, ILEOPLASTY AND SACROPLASTY IN THE TREATMENT OF BONE LYTIC MULTIPLE MYELOMA: INITIAL EXPERIENCE.}

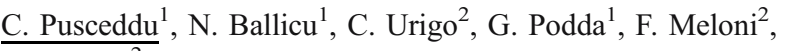 \\ G. Meloni ${ }^{2}$ \\ ${ }^{1}$ Oncology Hospital, Cagliari, Italy; ${ }^{2}$ Radiology Department of \\ University, Sassari, Italy
}

\section{PURPOSE}

To evaluate feasibility and safety of percutaneous femoroplasty, ilioplasty and sacroplasty in the treatment of painful osteolytic multiple myeloma located in critical area to high risk of pathological fracture.

\section{METHOD AND MATERIALS}

Seven patients ( 2 men and 5 women, mean age 68 years), afected by multiple bone myeloma localizations, have been treated with percutaneous injection of polymethyl methacrylate (PMMA) into the supracetabular ( 3 cases), sacral ( 2 cases) and femoral bone cavity ( 2 cases). The lesion approach was performed using a 10-gauge bone biopsy needle under CT and fluoroscopic guidance. Subsequently, under fluoroscopic guidance the PMMA was injected. In a patient with large oteolityc defect of the head and neck of right femur the tip of the needle is inserted whit percutaneous transtrochanteric approach. The procedures was performed using local anaesthesia and conscious sedation in all patients.

RESULTS

Good technical success was achivied in all patients. No major complications were noted. Osteoplasty was performed using from 8 to $22 \mathrm{ml}$ of PMMA. Complete pain relief ocurred whitin 1 month in 6/7 patients and a significant decrease in the visual analogue scale score occurred in other patient. During the mean follow-up of 11 months (2-27 months) none of the patients developed pathological fracture in the inerventional site.

\section{CONCLUSION}

Percutaneous osteoplasty of the pelvis and femora is feasible and safe in patients affected by multiple myeloma bone lesions. The acrylic cement injection is a minimally invasive procedure providing immediate pain relief. Furthermore it contributes to reinforce the bony structure with prevention of pathological fractures and improvement of walking ability.

\section{PO2:4}

\section{SPINAL DURAL ARTERIOVENOUS \\ FISTULAS-THE SAFETY AND EFFECTIVENESS OF ENDOVASCULAR EMBOLISATION.}

P. Cowley, K. Collison, S. Brew

${ }^{1} \mathrm{NHNN}$, Queen Sq, London, United Kingdom

\section{PURPOSE}

To show that glue embolisation of spinal dural arterio-venous fistulas (SDAVF) is a justifiable first line treatment in selected patients.

\section{METHOD}

Between 2002 and 2006, 10 patients were diagnosed with SDAVFs in our institution. Of these 9 were assessed as suitable for endovascular embolisation. No significant complications were experienced by the patients and none of the treated lesions have recurred so far. The technical and clinical follow up data will be presented.

A meta analysis of the data published on several larger studies found in the literature since 2000 will be presented.

RESULTS

The reported suitability and success rate is quite variable. However, there is a clear trend towards greater effectiveness of the endovascular approach and there is widespread agreement on the safety of this approach in selected patients.

\section{CONCLUSION}

Our results, and those in the literature suggest that glue embolisation of SDAVFs is a safe and justifiable first line treatment in selected patients. 


\section{Stroke}

\section{PO3:1}

ABSTRACT WITHDRAWN

PO3:2

\section{ABSTRACT WITHDRAWN}

\section{PO3:3}

\section{THE SONOGRAPHIC PITFALL OF CAROTID COLLATERALIZATION VIA VASA VASORUM}

J. Soares-Fernandes, M. Ribeiro, Z. Magalhães, R. Maré, E. Lourenço, J. Rocha

${ }^{1}$ Hospital S. Marcos, Braga, Portugal

\section{INTRODUCTION}

Collateralization through vasa vasorum of an atheromatous occlusion of the ICA is a rare finding. Growth of collaterals in the vessel's wall is a slow process, stimulated by the proangiogenic properties of the plaque. Differentiating carotid occlusion with collateralization from pre-occlusive stenosis is of the utmost importance because there is no benefit of endarterectomy or angioplasty in the first situation.

CASE REPORT

A 52-year-old man, with obesity, type 2 diabetes mellitus and hypertension, was admitted because of sudden onset of left hemiparesis and somnolence. Brain MRI disclosed a recent right thalamic stroke and multiple encephaloclastic lesions, suggestive of vascular sequelae. Carotid ultrasound demonstrated occlusion of the right internal carotid artery (ICA) and residual flow in the periphery of the left ICA lumen. Subocclusive stenosis was suspected. Carotid arteriography confirmed atherosclerotic occlusion of both bulbar ICAs. On the left side, however, filiform collateralization with a spiral configuration was seen, extending from the ICA bulb to the cavernous segment. The patient was offered medical treatment.

\section{DISCUSSION}

Carotid ultrasound is a reliable method in the assessment of ICA stenosis and occlusion. However, the findings of residual flow with normal velocities and waveform may erroneously be attributed to subocclusive carotid disease, in which has been shown that velocity measurements start to decrease. Therefore corroboration with arteriography is recommended. High-resolution transverse color-coded sections showing segments of flow within the ICA wall itself, outlining the vessel's circumference may be a clue to the correct diagnosis.

\section{PO3:4}

\section{DIFFUSION-WEIGHTED IMAGING IN CEREBRAL HEMORRHAGIC LESIONS}

V. Katsaros ${ }^{1}$, S. Lyra ${ }^{2}$, K. Bakopoulos ${ }^{3}$, A. Papatheodorou ${ }^{2}$, A. Katsarou ${ }^{2}$, N. Batakis ${ }^{2}$

${ }^{1}$ Department of CT and MRI, IKA Oncology Hospital, Athens, Greece; ${ }^{2}$ Department of Radiology, Red Cross Hospital, Athens, Greece; ${ }^{3}$ Department of Neurosurgery, Red Cross Hospital, Greece

\section{PURPOSE}

To evaluate diffusion weighted imaging (DWI) in cerebral hemorrhagic lesions.

\section{MATERIAL AND METHODS}

The last year we examined 12 patients ( 2 female, 10 male) with cerebral hemorrhagic lesions. The MRI protocol used includes axial, coronal and sagittal T1-, T2-weighted sequences, FLAIR, T2* and DWI $(b=0, b=500, b=1000$, ADC map), as well as contrast enhanced T1-weighted images.

\section{RESULTS}

We have found in the acute phase of hemorrhage 1 patient with decreased signal in DWI $(b=1000)$ and increased ADC compared to normal white matter and 3 patients with decreased signal in DWI and ADC. In the early subacute phase we found increased signal in DWI $(b=1000)$ and decreased ADC (2 patients), as well as decreased DWI and increased ADC (1 patient). In the late subacute phase and early chronic phases haemorrhagic lesions had isointense DWI $(b=1000)$ and increased ADC (1 patient) and increased DWI and decreased ADC (3 patients). In chronic phase 1 patient showed increased DWI and ADC.

\section{CONCLUSION}

DWI can afford information about hemorrhagic lesions of the brain and in combination with the other sequences establish the correct diagnosis, although in some cases it is inconclusive about the phase of the hemorrhage. DWI is accordant to T2 weighted images in imaging cerebral hemorrhagic lesions.

\section{PO3:5}

\section{HYPERTENSIVE ENCEPHALOPATHY: REVERSIBLE AND IRREVERSIBLE PATTERNS}

I. Cravo, T. Palma, C. Conceição, C. Gonçalves, P. Evangelista Hospital Fernando Fonseca, Lisbon, Portugal

\section{PURPOSE}

Magnetic resonance imaging (MRI) establishes the diagnosis of hypertensive encephalopathy (HE) or posterior reversible encephalopathy syndrome (PRES) associated with an increasing number of medical conditions. In HE T2-weighted images demonstrates hyperintensity on the posterior and anterior brain regions. In the acute setting, diffusion-weighted imaging (DWI) permits the accurate diagnosis, distinguishing ischemic injury from vasogenic brain edema and guiding the therapeutic decisions and evaluating the prognostic outcome. 


\section{METHODS}

Serial MRI studies of 9 patients with previously described risk factors for HE was analyzed. The MRI protocol included fluid attenuated inversion recovery (FLAIR) weighted images, diffusion-weighted imaging (DWI) and apparent diffusion coefficient (ADC) maps. All patients had acute neurological symptoms (headaches, seizures, visual disorders and vigilance disturbances) together with detectable hypertensive crises.

\section{RESULTS}

MRI showed abnormal T2-weighted/FLAIR signal intensity mostly in the posterior cerebrum but also in the frontal and temporal lobes, brain stem, cerebellum and thalamus. The hyperintensity on T2-weigthed /FLAIR images and on ADC maps of the lesions were consistent with vasogenic edema. When the blood pressure was normalized, reversibility of these lesions was detected and was considered as proof of diagnosis. In three patients that had poor outcome, MRI showed more extensive T2weighted/FLAIR brain abnormalities and some foci of DWI hyperintensities with decreased ADC maps, consistent with ischemia, status epilepticus and brain haematoma, respectively. CONCLUSION

DWI and ADC maps can differentiate reversible HE brain lesions from permanent brain tissue injury, playing an important role on the different therapeutic and prognostic consequences.

\section{PO3:6}

MULTIMODAL MORPHOLOGICAL AND PHYSIOLOGICAL SERIAL BRAIN MR IMAGING IN MELAS: ELEMENTS FOR NEW AND MORE THOROUGH UNDERSTANDING OF THIS METABOLIC ENCEPHALOPATHY

\author{
A. Cianfoni ${ }^{1}$, S. Servidei ${ }^{2}$, R. Calandrelli ${ }^{1}$, \\ T. Tartaglione ${ }^{1}$, G. Di Lella ${ }^{1}$, A. Cerase ${ }^{3}$, \\ C. Colosimo ${ }^{1}$ \\ ${ }^{1}$ Dept. Bio-Images Radiological Sciences - Catholic University - \\ Policlinico Gemelli - Rome, Rome, Italy; \\ ${ }^{2}$ Dept. Neurology - Catholic University - Policlinico Gemelli - \\ Rome, Rome, Italy; ${ }^{3}$ Neuroradiology Uoc- Policlinico Santa Maria \\ Delle Scotte, Siena, Italy
}

MELAS (mitochondrial encephalomyopathy, lactic acidosis, stroke-like events) is a mitochondrial disorder characterised by sudden neurological deficits (SND), similar to vascular strokes. Mitochondrial angiopathy and metabolic neuronal dysfunction might be the cause of stroke-like events. MELAS brain lesions are generally reported as displaying reversible vasogenic edema, but DWI added new elements of understanding.

During 3 years we studied 3 consecutive patients (F/M:2/1, age range 26-54 years) with MELAS A3243G-mutation and SND. MRI included FSE T2-W, FLAIR, DWI, 3D TOF MRA, enhanced T1$\mathrm{W}$ images, and MR-spectroscopy (MRS). One patient underwent perfusion-MR (pMR). Apparent diffusion coefficient (ADC) maps were generated from DWI data. Patients underwent serial MRI, at admission, and in the following weeks, with follow-up at two years in 1 case
All patients showed large T2-bright lesions, with focal swelling, in supratentorial cortical and subcortical areas, with no vascular distribution. The lesions were DWI-bright, with patchy mixed pattern of restricted and increased diffusion on ADC maps. MRA showed no vascular occlusion. MRS found high Lactate and low NAA in the lesions, compared to contralateral hemisphere, used as control. The patient undergone pMR had signs of hyperperfusion in the affected regions. Follow-up MRI showed almost complete reversibility of the brain lesions, with residual scattered gyral enhancement, and subsequent development of patchy cortical gliosis. Restricted diffusion eventually turned into increased diffusion on ADC maps

Pathophysiology of SND in MELAS is still unclear. We observed signs of metabolic impairment, with lactate increase, patent vessels and hyperperfusion; restricted diffusion suggested presence of cytotoxic edema, but unlike in true ischemic infarcts, it was partially reversible. Only the most severely affected areas experienced bloodbrain-barrier disruption and gliotic changes. We suggest a reversible metabolic neuronal dysfunction as the primary mechanism of SND in MELAS; when the metabolic impairment is very severe or prolonged, neuronal death and irreversible changes may occur.

\section{PO3:7}

\section{DIFFUSION AND PERFUSION IMAGING IN PATIENTS WITH TRANSIENT ISCHEMIC ATTACK}

A. Cabrera Zubizarreta ${ }^{1}$, I. Saralegui Prieto ${ }^{1}$, I. Vicente Olabarria ${ }^{1}$, E. Astigarraga Aguirre $^{1}$, E. Ferreira Gonzalez Viñaspr ${ }^{2}$, M. Gomez Beldarrain $^{2}$, B. Fernandez-Ruanova ${ }^{1}$

${ }^{1}$ Osatek, Galdakao, Spain; ${ }^{2}$ Neurology Department, Galdakao, Spain

\section{PURPOSE}

To evaluate the usefulness of diffusion weigthed imaging (dwi), perfusion weigthed imaging (pwi) and magnetic resonance angiography (mra) in patients with transient ischemic attack (tia) and to correlate the findings with the patients clinical symptoms. METHODS

We prospectively evaluated 51 patients $(31 \mathrm{~m} / 20 \mathrm{f})$ ages ranging from 34 to 86 years with clinical diagnosis of tia. All examinations were performed in the first 48 hours after the clinical event.

Exams were performed in a $1.5 \mathrm{t}$ system. Besides the standard mr imaging dwi $(b=1000 ; b=3000)$ and pwi were obtained in all patients. $0.2 \mathrm{ml} / \mathrm{kg}$ of gadolinium at a rate of $5 \mathrm{ml} / \mathrm{sec}$ was administered with the use of a power injector and mtt maps were calculated. Gadolinium enhanced mra was performed in 37 patients. RESULTS

T2 and flair correctly diagnosed $66,67 \%$ and $74,5 \%$ of the cases, considering $\mathrm{dw}$ as the gold standard. Interobserver agreement was excellent for dwi $(\hat{\mathrm{e}}=1,00)$ and moderate for $\mathrm{t} 2(\hat{\mathrm{e}}=0,45)$ and flair (ê $=0,52)$.

Dysarthria was the most common clinical finding. Dw was positive in 22 patients (46\%). The median duration of clinical symptoms was 30 minutes $(10-180 \mathrm{~min})$ for patients with positive dwi and 120 min for patients with negative dw. Dw was positive in $46 \%$ of patients with aphasia $(5 / 11), 47 \%$ of patients with motor 
syndrome (9/19), and $33 \%$ in patients with sensitive (3/9) and motor-sensitive syndrome (2/6), without statistical differences among groups.

Pwi demonstrated perfusion delay in $29,5 \%$ of the patients(14/51), one of which had a negative dwi. In 13 patients the oligohemic area was larger than the dwi lesions.

No correlation was demonstrated between the clinical diagnosis and the presence of stenosis.

$21.5 \%$ of the patients recurred during the first year but no difference was found between patients with positive and negative dwi.

\section{CONCLUSION}

Dwi and pwi can be included in the imaging protocol of patients with tia in order to correctly classify them as stroke.

\section{PO3:8}

\section{ANALYSIS OF A UNIFORM COHORT OF 398 PATIENTS STUDIED BY CT ANGIOGRAPHY OVER 24 MONTHS: STENO-OCCLUSIVE CAROTID ARTERY DISEASE. EPIDEMIOLOGICAL DIAGNOSTIC CONSIDERATIONS}

\author{
C. Barbara ${ }^{1}$, A. Stafa ${ }^{2}$, L. Simonetti ${ }^{1}$, L. Pedrini ${ }^{3}$, \\ M. Leonardi ${ }^{2}$ \\ ${ }^{1}$ Neuroradiology, Maggiore Hospital, Bologna, Italy; \\ ${ }^{2}$ Neuroradiology, Bellaria Hospital, Bologna, Italy; \\ ${ }^{3}$ Vascular Surgery, Maggiore Hospital, Bologna, Italy
}

\section{BACKGROUND}

Multislice CT scanners are playing an increasingly important role in the study of steno-occlusive carotid artery disease prior to treatment.

We used a large uniform cohort of patients to assess the diagnostic potential of multislice CT imaging and analyse the epidemiological findings.

\section{PATIENTS AND METHODS}

From February 2005 to February 2007 CT angiography was performed in 714 patients with carotid arteriosclerotic disease aged between 58 and 83 years (average 72). From this cohort we isolated 398 patients with the same geographic origin (Bologna and province) and clinical referral information (initial ultrasound diagnosis of stenosing carotid artery disease).

Scans were performed on a multibank 40 slice system with synchronized injection of $50 \mathrm{ml}$ water soluble contrast medium (iopamidol) containing $370 \mathrm{mg} / \mathrm{ml}$ iodine at a flow rate of $4 \mathrm{ml} \mathrm{a}$ second.

Echo-doppler findings were doubtful but indicated TEA on the basis of clinical findings, age, plaque features or patient choice in 102 patients who underwent CT scan of the supra-aortic trunks and intracranial circulation (average volume examined represented by 315 axial scans).

Echo-doppler findings were doubtful but showed indications for treatment or type of treatment (endovascular or TEA) in 296 patients who underwent CT scan of the aortic arch, supra-aortic trunks and intracranial circulation (average volume examined represented by 475 axial scans).

\section{RESULTS AND CONCLUSIONS}

The results are still being processed, analysing:

- Epidemiological data (age, sex, correlated disease, symptoms);

- Reliability of plaque typing in operated patients;

- Determination of stenosis in cases with doubtful ultrasound findings;

- Incidence of significant intracranial abnormalities, namely associated intracranial stenoses, aneurysms and circle of Willis variants likely to influence the choice of treatment.

\section{PO3:9}

\section{BIPHASIC WORSENING INTRACEREBRAL BLEEDING. INCIDENCE AND POSSIBLE PREDICTIVE RISK FACTORS}

$\underline{\text { C. Barbara }}^{1}$, D. Rossi ${ }^{2}$, G. Procaccianti ${ }^{3}$, F. Cancellieri ${ }^{4}$, L. Simonetti ${ }^{1}$

${ }^{1}$ Neuroradiology, Maggiore Hospital, Bologna, Italy;

${ }^{2}$ Neurosurgery, Maggiore Hospital, Bologna, Italy; ${ }^{3}$ Stroke Unit, Maggiore Hospital, Bologna, Italy; ${ }^{4}$ Reanimation, Maggiore Hospital, Bologna, Italy

\section{BACKGROUND}

Spontaneous intracerebral haemorrhage is usually considered a monophasic event whose neuroradiological follow-up serves to monitor any perilesional oedema and its progressive reabsorption. More recent literature has focused on biphasic bleeding with CT features worsening within the first 24 hours. This finding has raised the problem of serial follow-up CT scans after the initial diagnosis both for prognostic purposes and to provide evidence of the lesion reaching a volume justifying surgical evacuation according to STICH criteria. This study aimed to ascertain the incidence of biphasic bleeding and the association of predictive risk factors in our experience over the past two years.

\section{PATIENTS AND METHODS}

In the last two years we have examined 192 patients with intracerebral haemorrhage referred directly by our emergency room or transferred from other hospitals. All patients underwent CT scan for initial diagnosis and all except seven (who died in the first hours) had a follow-up CT scan between ten and 96 hours after stroke.

\section{RESULTS AND CONCLUSIONS}

The incidence of increase in the volume of the bleed at the second CT scan was $16 / 185$, equal to $8.6 \%$.

The findings in our cohort are currently being processed to disclose any correlated risk factors which could predict the population at greatest risk for whom CT follow-up scan could be indicated six to 12 hours later. 


\section{PO3:10}

\section{QUANTITATIVE DETERMINATION IN THE CT DIAGNOSIS OF ISCHAEMIC AND HAEMORRHAGIC STROKE}

C. Barbara $^{1}$, D. Rossi ${ }^{2}$, G. Procaccianti ${ }^{3}$, L. Simonetti $^{1}$,

M. Leonardi ${ }^{4}$

${ }^{1}$ Neuroradiology Maggiore Hospital, Bologna, Italy;

${ }^{2}$ Neurosurgery Maggiore Hospital, Bologna, Italy; ${ }^{3}$ Stroke Unit Maggiore Hospital, Bologna, Italy; ${ }^{4}$ Neuroradiology Bellaria Hospital, Bologna, Italy

\section{BACKGROUND}

Quantitative determination of ischaemic and haemorrhagic stroke yields important information for the purposes of instituting appropriate treatment.

In ischaemic stroke treatment depends on the patient's ASPECT score, especially when intravenous thrombolysis is indicated.

In haemorrhagic stroke the STICH trial found that the indication for surgical evacuation depends on the volume of ICH and its location. We present our experience in the last two years focusing on the standardization and simplification of methods quantifying stroke damage.

\section{PATIENT AND METHODS}

Patients comprised cases presenting at the emergency room with a diagnosis of stroke.

An initial period of training, also involving clinicians, focused on how to read the CT report in ischaemic stroke not only in terms of topographic brain findings, but also the vascular territory involved. Subsequently the ASPECT score was assigned checking the accuracy of the score in long-term follow-up examinations. The aim of this exercise was the forthcoming implementation of the project "intravenous and intra-arterial thombolysis in cerebral stroke" in the second half of 2007.

Attempts have been made to establish an extreme simplification of the volumetric ICH assessment to allow a realistic routine application of the score. The paper discusses the basis of this simplification and results.

\section{PO3:11}

\section{DOPPLER ULTRASOUND ASSESSMENT OF THE STENTED CAROTID ARTERY}

\section{A. Afaq ${ }^{1}$, G. Rajeswaran ${ }^{2}$}

${ }^{1}$ Hammersmith Hospitals NHS Trust, London, United Kingdom; ${ }^{2}$ Chelsea and Westminster Hospital NHS Trust, London, United Kingdom

\section{PURPOSE}

Although controlled trials continue to evaluate the role of carotid artery stenting against the gold standard of endarterectomy, the patient population with stented carotid arteries is expanding rapidly. The biomechanical properties of the carotid arteries are altered following angioplasty and stent placement which significantly influences the evaluation with ultrasound.
Few studies, with relatively small patient numbers have been conducted to analyse sonographic assessment of the stented carotid artery. A review of these studies and the current literature is necessary to consider current opinions on this, increasingly important topic. MATERIALS AND METHODS

A Medline search and analysis was performed on all English language articles on the ultrasonographic assessment of the stented carotid artery.

RESULTS

Published studies over the last 10 years provide conflicting reports on the efficacy of ultrasound follow up of the stented carotid artery. A number of features contribute to the differences on Doppler assessment including reduced compliance, fracturing of the carotid plaque rather than removal and highly sonographically reflective material within the carotid artery. The most significant consequence is the challenge of the previously determined velocity criteria to assess degrees of stenosis. Factors which may affect carotid velocities include aortic stenosis, arrythmias, acute stroke, contralateral occlusion and severe distal carotid artery disease.

\section{CONCLUSION}

After stenting, the physical properties of the carotid arterial wall have the greatest impact on blood flow velocities at mild and moderate degrees of stenoses. Several authors have proposed increased peak systolic velocity values and internal carotid artery/common carotid artery ratios to identify carotid stenoses in these patients.

Normal stented carotid arteries and severe in-stent stenosis can be reliably demonstrated on ultrasound. However, larger studies with longer follow up are necessary to demonstrate which of the velocity criteria are most appropriate for mild and moderate stenosis in the stented carotid artery.

\section{PO3:12}

RIGHT-TO-LEFT SHUNT IN MIGRAINE AND WHITE MATTER LESIONS: CONVENTIONAL AND DIFFUSION MRI STUDY

R. Parodi ${ }^{1}$, L. Roccatagliata ${ }^{2}$, L. Bonzano ${ }^{2}$, M. del Sette ${ }^{3}$, L. Castellan ${ }^{1}$

${ }^{1}$ Neuroradiology Department, San Martino University Hospital, Genoa, Italy; ${ }^{2}$ Magnetic Resonance Research Centre on Nervous System Diseases, University of Genoa, Genoa, Italy; ${ }^{3}$ Department of Neurosciences, Ophtalmology and Genetic - University of Genova, Genoa, Italy

\section{PURPOSE}

To evaluate white matter lesions (WML) by MRI in migraineurs with aura and to correlate WML with the presence of right-to-left shunt (RLS) and with clinical characteristics of migraine patients. METHODS

Eighty-seven consecutive subjects admitted for migraine with aura were evaluated. The patients were screened for migraine characteristics, cerebrovascular risk factors and thrombophylic states. We used transcranial Doppler sonography to diagnose RLS, conventional T2 weighted MRI and diffusion weighted imaging (DWI)to evaluate WML. 


\section{RESULTS AND CONCLUSIONS}

WML were present in $61 \%$ of patients on T2 weighted images. We did not detect any DWI hyperintense lesion; RLS was present in $45 \%$ of patients. Subjects with WML were older $(\mathrm{p}<.001)$ and had higher Body Mass Index $(\mathrm{p}<.01)$. There was no significant difference in the total volume of WML (lesion load) within WML in the group with and without RLS. At multivariate analysis, only the number of attacks with aura significantly correlated with lesion load $(p=0.03)$. In migraine with aura white matter lesion load do not depend on the presence of RLS but are significantly associated to the frequency of migraine with aura attacks.

\section{Advanced Imaging Techniques (Diffusion, Perfusion, Spectroscopy, Functional)}

\section{PO4:1}

\section{EVALUATION OF THERAPEUTIC EFFECT OF SURGERY FOR COMPRESSIVE CERVICAL MYELOPATHY BY USING FIBER TRACKING BASED ON LINE SCAN DIFFUSION TENSOR IMAGING.}

A. Wada, K. Oda, T. Hayashi, T. Katsube, S. Hara, T. Yoshizako, N. Uchida, H. Kitagaki

Shimane University Faculty of Medicine, Izumo, Japan

\section{PURPOSE}

We exhibit the finding change on line scan diffusion tensor MR imaging (DTI) in association with the surgical treatment effect of compressive cervical myelopathy.

METHODS

Diffusion tensor MR imaging of cervical spinal cord was performed at pre- and post-surgery in seven cervical compression myelopathy patients ( 3 males, 4 females, average age 70.7 y.o.). DTI changes were compared with improvement of Japanese Orthopedic Association (JOA) score (highest score; 17 points) which was a clinical index of severity of cervical myelopathy. All MR examination was performed on 1.5 T MR unit (Signa Cvi; GE) with neurovascular coil and line scan sequence was applied to diffusion tensor imaging; TR/ TE: 3696-4092/55-59 msec, FA: 90, NEX: 1, FOV 24×24 cm, acquisition matrix: $128 \times 128$, effective section thickness/gap: $3 /$ $0 \mathrm{~mm}$, voxel size: $1.875 \times 1.875 \times 3.0 \mathrm{~mm}, \mathrm{~b}$ factors: 5 and $1000 \mathrm{~s} /$ $\mathrm{mm} 2$, total scan time: 18 minutes. Diffusion tensor analysis was performed with VOLUMEONE Ver.1.72 and dTVIISR which were free software developed by University of Tokyo. Tractography with seed area at upper intervertebral level and end area at lower intervertebral level was evaluated.

RESULTS

All patients revealed improvement of Japanese Orthopedic Association (JOA) score ; pre- and postoperative average JOA score was 9.2(5-11.5) and 12.4 (7.5-15). On DTI analysis, fractional anisotrophy at the decompressive cervical cord level improved after decompression and the visualization of the nerve fibers which passed the compressed level was improved on Tractography. Regardless of persistence of intramedullary high signal intensity area on conventional T2-weighted images, the improvement of nerve fiber visualization was recognized with postoperative improvement of JOA score.

CONCLUSION

DTI of cervical spinal cord reflects surgical therapeutic effect of compressive cervical myelopathy.
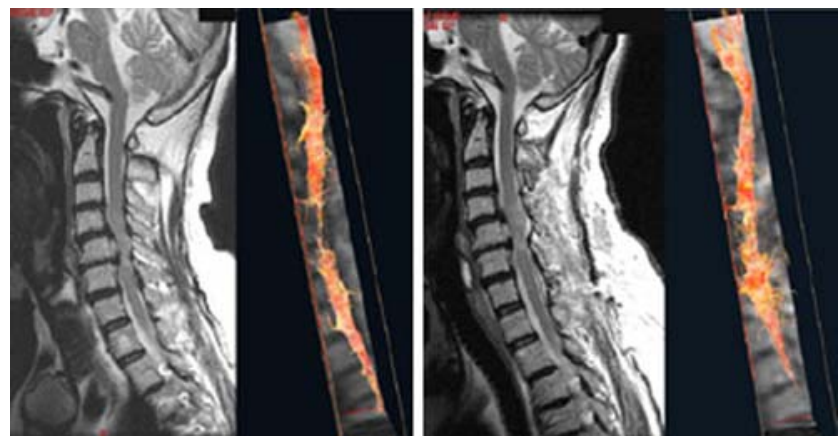

PO4:2

\section{CT-PERFUSION SHOWS DECREASED CBF AND NORMAL CBV IN MOYAMOYA SYNDROME}

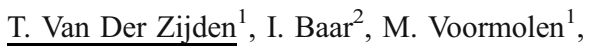
T. Menovsky ${ }^{3}$, L. Van Den Hauwe ${ }^{1}$, J. Van Goethem ${ }^{1}$, P. Cras ${ }^{2}$, P. Parizel ${ }^{1}$

${ }^{1}$ Department of Radiology, Antwerp University Hospital Antwerp Belgium; ${ }^{2}$ Department of Neurology, Antwerp University Hospital Antwerp Belgium; ${ }^{3}$ Department of Neurosurgery, Antwerp University Hospital Antwerp Belgium

\section{PURPOSE}

Moyamoya syndrome is a rare cerebrovascular condition. Our goal is to review the imaging features (conventional angiography, MRI, CT-angiography) and to document the added value of CTperfusion imaging in a patient with Moyamoya syndrome.

\section{PATIENT AND MATERIALS}

A 37-year old woman of Korean origin presented with left-sided hemianopsia, scotomas, hypoesthesia and chronic right-sided headache. Previous medical history was unremarkable, except for cigarette smoking. A noncontrast cerebral CT-scan revealed lacunar infarction of the right internal capsule anterior limb. CTangiography, MRI and conventional angiography showed severe central vasculitis in accordance with Moyamoya syndrome. The right internal carotid artery tip and the proximal anterior (ACA) and middle cerebral artery (MCA) segments were stenotic, with collateral circulation to the (relatively normal) peripheral cerebral arteries from hypertrophic lenticulostriate and thalamoperforating arteries. To a lesser extent, similar intracranial vasculitis features were found on the left. Cerebral CT-perfusion (64-detector row) revealed diminished cerebral blood flow $(\mathrm{CBF})$ with prolonged mean transit time (MTT) in the right MCA distribution territory. Conversely, cerebral blood volume (CBV) was normal to slightly elevated in the affected right cerebral hemisphere. The patient was 
treated surgically with several right-sided cranial burr-holes to stimulate neovascularization (ref.: Kawaguchi T, et al. Multiple burr-hole operation for adult moyamoya disease. J Neurosurg 1996;84:468-476).

\section{RESULTS AND CONCLUSIONS}

1. Moyamoya syndrome is an rare disorder of unknown pathogenesis characterized by progressive intracranial vascular stenoses of the circle of Willis.

2. Radiologic features include: stenosis of the supraclinoid internal carotid artery and basal teleangiectasias consisting of dilated collateral branches of the lenticulostriate and thalamoperforating arteries.

3. Parametric CT-perfusion maps reveal diminished CBF with preserved $\mathrm{CBV}$, indicating that the mechanism of cerebral autoregulation is still functional. We surmise that $\mathrm{CT}$ perfusion can play a role in the pre- and postoperative evaluation of patients with Moyamoya syndrome.

\section{PO4:3}

\section{EXCITOTOXICITY FOR DUMMIES}

${ }_{\text {L. Van Den Hauwe }}{ }^{1}$, P. Parizel ${ }^{2}$, E. Pelzers ${ }^{1}$, J. Van Goethem ${ }^{2}$ ${ }^{1}$ AZ KLINA, Brasschaat, Belgium; ${ }^{2}$ University Hospital Antwerp, Antwerp, Belgium

\section{PURPOSE}

Diffusion-weighted imaging (DWI) is a very sensitive technique for the early detection of ischemic stroke, due to its sensitivity for the detection of cytotoxic edema. However, there are several other neurological disorders which can also be associated with cytotoxic edema. It is now considered that glutamate-mediated "excitotoxic brain injury" is the final common pathway leading to cytoxic edema. In this pictorial essay, we shall discuss DWI abnormalities in a variety of conditions associated which excitotoxic brain damage.

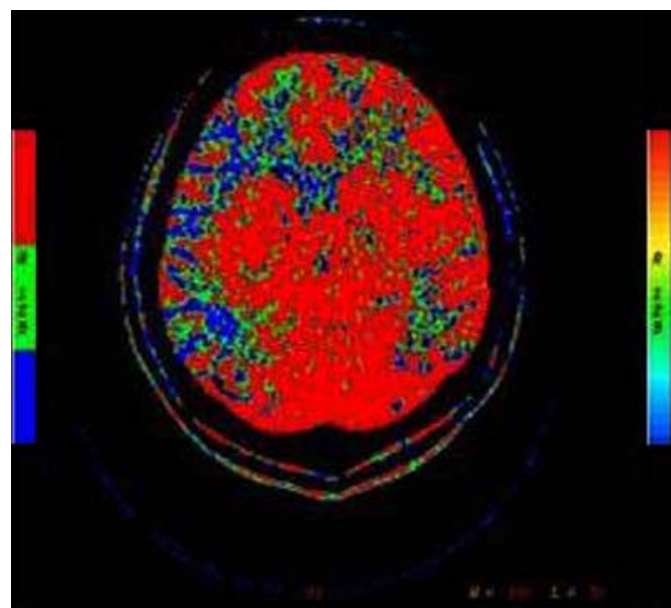

Bloodflow

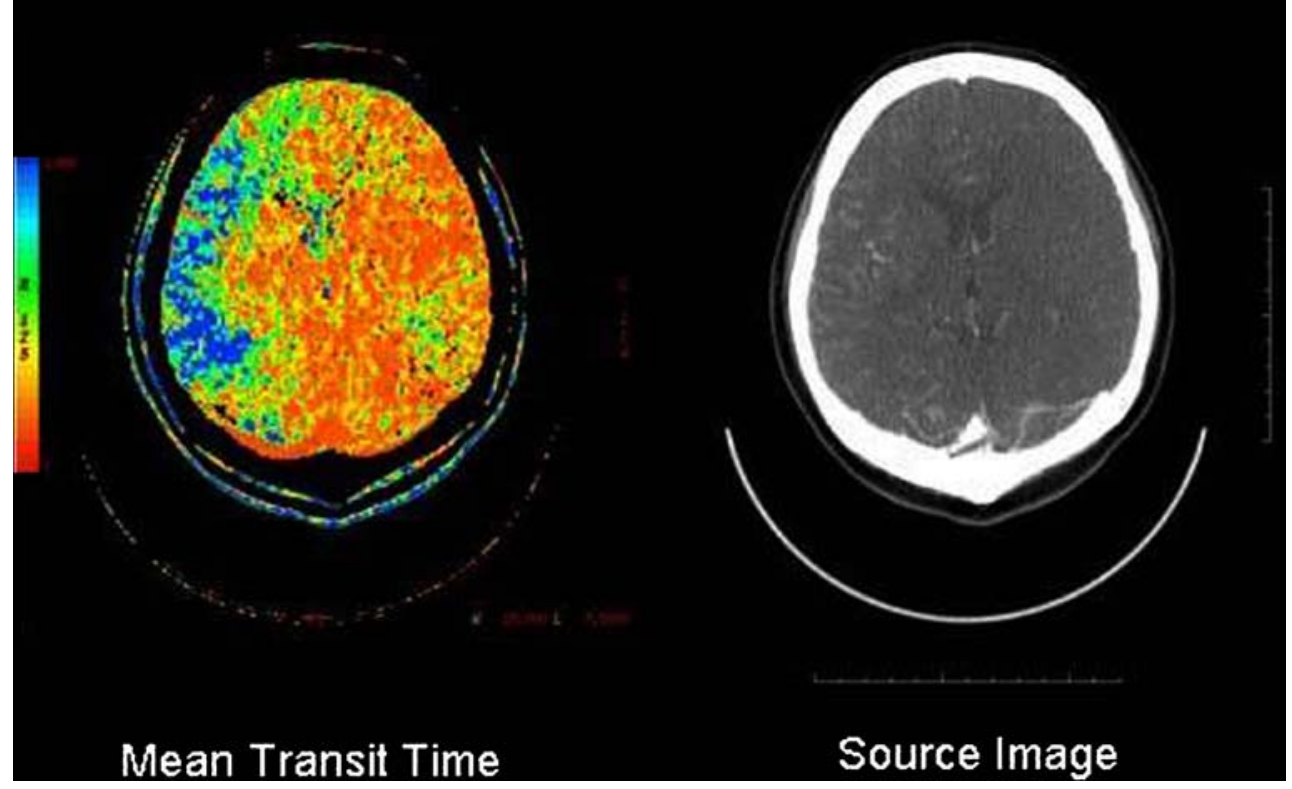




\section{MATERIALS AND METHODS}

We present a retrospective study. Over a two-year period, all patients with abnormal DWI were collected, and acute ischemic stroke syndromes were excluded. Also excluded were patients in whom diffusion restriction was caused by increased cellularity (e.g. bran tumors, lymphoma, etc.) or by increased viscosity (bacterial abscess, hemorrhage, epidermoid cysts, etc.). Selected patients are demonstrated in this scientific exhibit. We performed an extensive literature search.

\section{RESULTS}

Pathological conditions included: hypoxic ischemic encephalopathy, status epilepticus, herpes encephalitis, Creutzfeldt-Jacob disease, shaken baby syndrome, diffuse axonal injury, demyelinating diseases such as multiple sclerosis and acute disseminated encephalomyelitis. CONCLUSION

The take-home message is that cytotoxic edema does not only occur in ischemic stroke, but can be found in a wide variety of neurological diseases. It is surmised that increased levels of glutamate (due to increased release, decreased reuptake and/or leakage due to disruption of axonal membranes) in the synaptic cleft lead to transsynaptic excitotoxic brain injury. Radiologists should be familiar with this phenomenon, to avoid that differential diagnosis is narrowed just to that of ischemic stroke.

\section{PO4:4}

\section{THE EFFECT OF ETHYL ALCOHOL ON THE CENTRAL NERVOUS SYSTEM DETERMINED WITH THE HMRS TECHNIQUE}

\section{A. Urbanik, J. Kozub, B. Sobiecka, I. Herman-Sucharska}

Department of Radiology, Medical College, Jagiellonian University, Kraków, Poland

\section{PURPOSE}

The objective of the research was to assess the effect of ethyl alcohol on the central nervous system with the HMRS technique. METHODS

The research, performed with the MRI scanner, covered 14 male volunteers. The experiment consisted of four sessions: the first prior to drinking alcohol and $0.5 \mathrm{~h}, 1 \mathrm{~h}, 2 \mathrm{~h}$ after. All the volunteers drank $150 \mathrm{ml}$ of vodka. The dynamics of changes in the content of alcohol in the exhaled air was determined with an alcometer. The readings were taken in cerebellum, the occipital lobe, the subcortical structures, and the frontal lobes. The analysis was performed for $\mathrm{Lip} / \mathrm{Cr}, \mathrm{Eth} / \mathrm{Cr}, \mathrm{Lac} / \mathrm{Cr}, \mathrm{NAA} / \mathrm{Cr}, \mathrm{Gaba} / \mathrm{Cr}$, Glx1/Cr, Cho/Cr, Glc1/Cr, mI/Cr, Glc2/Cr, Glx2/Cr. RESULTS

A $1.2 \mathrm{ppm}$ peak was found in all the locations in the examined volunteers when compared with the initial spectra from the first session prior to drinking alcohol. Decrease in the relative proportion of concentrations of ethyl alcohol to creatine Eth/Cr was found in all the examined locations during time.

Moreover, distinct increase in $\mathrm{Lip} / \mathrm{Cr}$, Lac/Cr, Glc1/Cr, Glc2/Cr was found in the following sessions when compared with the first session.
Decrease in GABA/Cr, Glx1/Cr, Glx2/Cr was found, all in the following sessions.

\section{CONCLUSIONS}

HMRS is a method, which enables assessment of the effect of ethyl alcohol on the central nervous system.

On the basis of the conducted research, the following was found out:

1. Presence of ethyl alcohol in cerebral tissue on the basis of the visible peak $1.2 \mathrm{ppm}$, absent in the spectra from the first session prior to drinking alcohol.

2. The dynamics of changes in the relative proportion of concentrations of ethyl alcohol to creatine Eth/Cr corresponds with the changes in concentration of ethyl alcohol in the exhaled air during time.

3. The changes in maintaining the relative proportions in concentrations, especially for $\mathrm{Lip} / \mathrm{Cr}$, $\mathrm{Lac} / \mathrm{Cr}$ and $\mathrm{Glx} / \mathrm{Cr}$.

\section{PO4:5}

HMRS IMAGING IN RELAPSING-REMITTING MULTIPLE SCLEROSIS-BRAIN METABOLITE ABNORMALITIES IN CORTICAL GREY MATTER, THALAMIC GREY MATTER AND WHITE MATTER

A. Urbanik ${ }^{1}$, M. Szafirska ${ }^{1}$, I. Herman-Sucharska ${ }^{1}$, $\overline{\text { B. Sobiecka }}^{1}$, J. Kozub ${ }^{1}$, T. Róg ${ }^{2}$, E. Gryz-Kurek ${ }^{2}$

${ }^{1}$ Department of Radiology, Medical College, Jagiellonian University, Kraków, Poland; ${ }^{2}$ Institute of Neurology, Medical College, Jagiellonian University, Kraków, Poland

\section{PURPOSE}

In multiple sclerosis proton MRS can show abnormalities within the lesions. The aim of the study was the assessment of grey matter (cortical and) and white matter that appears normal on conventional MR imaging, using proton MRS.

MATERIALS AND METHODS

45 patients (17-48 years of age, mean 28 years, 32 women, 13 men) with relapsing-remitting multiple sclerosis were examined with the use of 1,5 T GE Signa Excite unite. T2-weighted MR imaging and Single Voxel Spectroscopy (SVS/PRESS) was performed. Absolute $\mathrm{N}$-acetylaspartate (NAA) creatine $(\mathrm{Cr})$, choline (Cho) and myoinositol $(\mathrm{mI})$ levels were obtained from normal-appearing white matter, cortical grey matter (from frontal region) and thalamic grey matter. Afterwards $\mathrm{NAA} / \mathrm{Cr}, \mathrm{Cho} / \mathrm{Cr}$ and $\mathrm{mI} / \mathrm{Cr}$ ratios were calculated. The results were compared with those of control group. RESULTS

The results for white matter, cortical grey matter and thalamic grey matter in patients with relapsing-remitting multiple sclerosis were different from the results of the control group. For the patients the following ratios were found:

- normal-appearing white matter $\mathrm{NAA} / \mathrm{Cr}=1,87+/-0,34$, Cho/ $\mathrm{Cr}=1,01+/-0,31, \mathrm{mI} / \mathrm{Cr}=0,74+/-0,35$

- cortical grey matter $\mathrm{NAA} / \mathrm{Cr}=1,95+/-0,45, \mathrm{Cho} / \mathrm{Cr}=0,88+/$ $-0,18, \mathrm{mI} / \mathrm{Cr}=0,91+/-0,24$

- thalamic grey matter $\mathrm{NAA} / \mathrm{Cr}=1,94+/-0,38, \mathrm{Cho} / \mathrm{Cr}=0,98+/$ $-0,2, \mathrm{mI} / \mathrm{Cr}=0,92+/-0,31$ 
In the control group, these ratios were:

- white matter $\mathrm{NAA} / \mathrm{Cr}=1,92+/-0,85, \mathrm{Cho} / \mathrm{Cr}=0,86+/-0,26$, $\mathrm{mI} / \mathrm{Cr}=0,58+/-0,19$

- cortical grey matter $\mathrm{NAA} / \mathrm{Cr}=2,41+/-0,78, \mathrm{Cho} / \mathrm{Cr}=1,22+/$ $-0,71, \mathrm{mI} / \mathrm{Cr}=0,97+/-0,62$

- thalamic grey matter $\mathrm{NAA} / \mathrm{Cr}=2,2+/-0,51, \mathrm{Cho} / \mathrm{Cr}=1,09+/$ $-0,26, \mathrm{mI} / \mathrm{Cr}=0,99+/-0,3$

\section{CONCLUSIONS}

The metabolic abnormalities in the patients with relapsing-remitting multiple sclerosis may be detected using HMRS. The changes are seen not only in lesions in white matter but also in normal-appeared white matter, cortical grey matter and in the thalamus.

\section{PO4:6}

\section{FRACTIONAL ANISOTROPY (FA) MEASUREMENT AT THE CEREBRAL PEDUNCLES: EARLY DETECTION OF WALLERIAN DEGENERATION IN PATIENTS WITH ACUTE STROKE}

F. Tsushima ${ }^{1}$, M. Nagahata ${ }^{1}$, S. Ono ${ }^{1}$, H. Seino ${ }^{1}$, $\overline{\text { K. Morimoto }}^{1}$, T. Ohata ${ }^{1}$, H. Miura $^{1}$, Y. Abe ${ }^{1}$, S. Kakehata ${ }^{2}$, K. Shibutani ${ }^{2}$, H. Midorikawa ${ }^{2}$, M. Ogawa ${ }^{3}$ ${ }^{1}$ Department of Radiology, Hirosaki University School of Medicine, Hirosaki, Japan; ${ }^{2}$ Department of Radiology, Aomori Prefectural Central Hospital, Aomori, Japan; ${ }^{3}$ Department of Neurology, Aomori Prefectural Central Hospital, Aomori, Japan

\section{PURPOSE}

Fractional anisotropy (FA) value is one of the rare absolute values in cerebral MR imaging. Purpose of this study is to evaluate the FA measurement of the cerebral peduncles for early detection of Wallerian degeneration in patients with acute cerebral infarction. METHODS

From June 2004 to May 2005, we performed scheduled MR examinations in five patients with acute cerebral hemispheric infarction resulting in complete hemiparesis. Magnetic resonance diffusion tensor imaging (DTI) was obtained on day 0, 3, 6, 10 and 14. Then we measured FA value of the bilateral cerebral peduncles. RESULTS

In four of the five patients, FA value of the affected cerebral peduncle was significantly reduced within 14 days after stroke, in comparison with the contralateral cerebral peduncle. On sequential MR examination, FA value of the affected cerebral peduncle tended to decline. Although the FA value of the affected side was not significantly lower than the contralateral cerebral peduncle on day 3, FA value of the affected peduncle was significantly lower on DTI obtained 6 days after stroke onset.

\section{CONCLUSION}

Conventional MR imaging without DTI needs 4 weeks interval between onset and MR examination to show a Wallerian degeneration of corticospinal tracts within the cerebral peduncle on MR images. With the use of DTI, Wallerian degeneration of the cerebral peduncle could be detected as a reduced FA value only
6 days after onset of the infarction. This result is compatible with previous histopathlogical reports.

\section{PO4:7}

\section{THE VARIATIONS OF WILLIS POLYGON ON MR ANGIOGRAPHY}

E. Kaymaz, U. Senol, K. Karaali, T. Sindel

Akdeniz University, Department of Radiology, Antalya, Turkey

\section{PURPOSE}

Brain MR angiography with TOF technique differs from other techniques which directly enhance vascular structures such as DSA. MR angiography is a noninvasive and useful method although its limited use. We aimed to investigate the variation of the Willis polygon and to determine the diameter of the intracranial arteries in healthy subjects with TOF technique METHODS

One hundred and ninety one healthy volunteers were included in the study. All of the volunteers underwent MR angiography with 3D TOF technique. Maximum intensity projection was performed. The variations and vessel diameters in age and sex groups were compared. Both reference and reconstructed images were used for the evaluation of the variations.

RESULTS

All of the variations of the Willis polygon described in the literature were demonstrated by MR angiography. There was no statistical difference in determining variations between reference and reconstructed images. The distribution of the Willis polygone variations on different age and sex groups as well as the diameter of the arteries are also given in the study.

\section{CONCLUSIONS}

TOF technique is effective for the demonstration of Willis polygon variations. Reconstructed images are as effective as reference images.

\section{PO4:8}

COMPUTER AIDED DIAGNOSIS OF EPILEPTOGENIC FOCI USING STATISTICAL PARAMETRIC MAPPING (SPM)

\section{B. Schmitz, A. Lehner}

Clinic for Diagnostic and Interventional Radiology, Ulm, Germany

\section{PURPOSE}

Epilepsy is a highly prevalent disease and surgery of epileptogenic foci is an increasingly important adjunctive therapy. However, epileptogenic foci can be very subtle on neuroimaging studies and observational oversight of lesions depicted by special MRI studies, but not recognized by the radiologist is not uncommon.

The purpose of the presented study is to develop and test a computer aided diagnostic tool with the aim to point the radiologist to certain spots of the patients brain which deviate from the signal intensity distribution of a normal population. 


\section{MATERIAL AND METHODS}

We retrospectively used high resolution brain scans $(1 \mathrm{~mm}$ isotropic resolution turbospinecho $\mathrm{T} 2$ scans) from a population of 20 healthy individuals to create a normal distribution of brain MRI signal intensities. Therefore scanned volumes were coregistered, normalized and smoothed and mean and standard deviation volumes were calculated using SPM (statistical parametric mapping, Wellcome Dept. of Cognitive Neurology, London, UK). In a second step brain scans from 20 patients with known epileptogenic foci were normalized and smoothed in the same way. Resulting brain volumes were subtracted voxel by voxel from the mean signal intensity volumes of the normal controls and divided by the standard deviation volume of the normal controls. The resulting volume was used as an overlay to the morphological patient scan and interactively thresholded to depict deviations from the normal control sample. The results of the computer aided diagnostic algorithm were judged by an experienced neuroradiologist.

\section{RESULTS}

All control scans were normalized without errors. However three patient scans with severe brain changes (Arnold Chiari II, mikrocephaly, severe hypoxia) could not be normalized with the SPM algorithm and had to be excluded from further examinations. The algorithm detected lesions like focal dysplasia (figure), tuber cinereum hamartoma, glioma or arachnoidal cysts at medium thresholds. To detect subtle changes like hippocampal sclerosis thresholds had to be lowered, rendering the algorithm less specific with more false positive results.

\section{CONCLUSION}

A computer aided diagnosis algorithm based on SPM algorithms to detect signal intensity deviations from a normal distribution is able to help the radiologist find a certain range of lesions. However, the experienced eye and expert knowledge of the neuroradiologist is irreplaceable in the differential diagnosis of epileptogenic brain lesions.

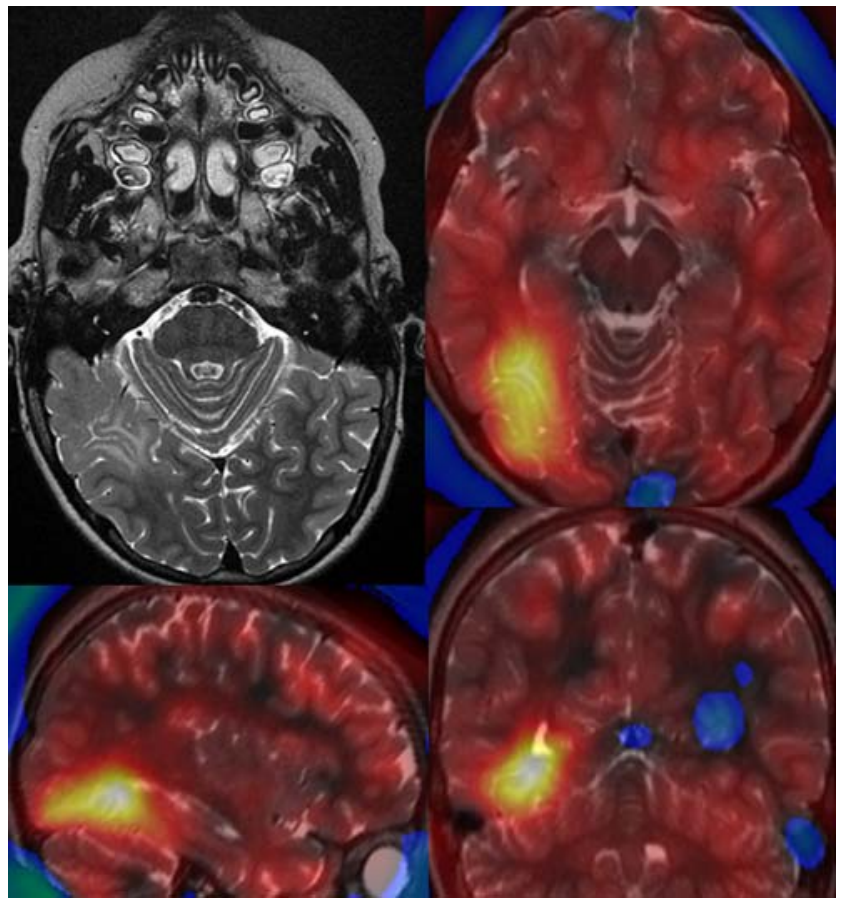

PO4:9

\section{CEREBRAL CT PERFUSION WITH EMPHASIS \\ ON PERMEABILITY SURFACE INDEX IN INTRACRANIAL SPACE OCCUPYING LESIONS.}

E. Mah Siew Ling, MBBS, MRAD, K. Rahmat, MBBS, FRCR, N. Ramli, MBBS, FRCR

University Malaya Medical Center, Kuala Lumpur, Malaysia

\section{BACKGROUND}

Radiologic findings of intracranial abscesses may mimic brain tumours and vice versa. By evaluating the Computed Tomography (CT) perfusion parameters especially the permeability surface, we aim to evaluate the usefulness of dynamic CT perfusion imaging as an alternative tool to differentiate brain tumours and intracerebral abscesses.

\section{METHODS}

A total of 26 patients underwent perfusion CT study were divided into 3 groups: Group 1, patients with primary brain tumours $(n=$ $10)$; group 2, patients with secondaries to the brain $(n=8)$ and group 3 , patients with cerebral abscesses $(n=8)$; mean perfusion parameters were obtained from the most enhancing part of the lesion. The relative ratios were calculated by using the results from mirrored regions within the contralateral hemisphere as reference.

\section{RESULTS}

There was significant difference in the relative cerebral blood flow (rCBF) and relative mean transit time (rMTT) values between the primary and secondary tumours $(\mathrm{p}<0.05)$. No significant difference was found in the relative cerebral blood volume (rCBV) and relative permeability surface index (rPS) values between these two groups $(p>0.05)$. When the values of primary and secondary tumours were combined and compared with those of cerebral abscesses, no significant difference in the rCBV, rCBF and rMTT values noted between cerebral tumours and cerebral abscesses ( $\mathrm{p}>$ $0.05)$. There was however, significant difference in the relative permeability surface values between intracranial tumours and cerebral abscesses $(p=0.002, p<0.05)$. By applying the receiver operating characteristics curve (ROC), a value of 25.11 for rPS was found to be the best estimate to discriminate cerebral tumours from cerebral abscesses with a sensitivity of $72.2 \%$. The false positive for this value is estimated to be $12.5 \%$

\section{CONCLUSION}

CT perfusion especially permeability surface index may allow the differentiation of cerebral abscesses from tumours making it a strong additional imaging modality in the early diagnosis of these two entities when conventional techniques fail.

\section{PO4:10}

CORRELATION OF CLINICAL SYMPTOMS, BRAIN METABOLITE RATIOS AND DIFFFUSION TENSOR IMAGING PARAMETERS IN THE SETTING OF FIBROMYALGIA

M. Petrou, B. Foerster, D. Clauw, P. Sundgren

University of Michigan, Ann Arbor, USA 


\section{PURPOSE}

Fibromyalgia is a common rheumatologic condition thought to be due to altered central nervous system processing of painful stimuli. The purpose of our study was to investigate the relationship between clinical symptoms in FM patients and metabolite ratios as well as DTI parameters in a number of brain regions implicated in pain processing.

\section{METHODS}

$21 \mathrm{FM}$ patients $(17$ female, 4 male, mean age $=41.0$ years $)$ underwent conventional structural brain MRI. Patients also underwent 2D-CSI MR spectroscopy, at the levels of the basal ganglia and centrum semiovale, using a point-resolved spectroscopy sequence (PRESS), TR/TE $=1500 / 144$. DTI was also performed using a single shot spin-echo EPI technique with $b=1000 \mathrm{~s} / \mathrm{mm} 2$ for each of 9 diffusion encoding directions and one $b=0$ image set. Standard metabolite ratios, fractional anisotropy (FA) and apparent diffusion coefficient (ADC) values were derived for a number of brain locations previously implicated in pain processing. Patients also underwent pain pressure testing and completed a number of selfreport questionnaires. Correlations between metabolite ratios, DTI parameters and clinical symptoms were investigated.

\section{RESULTS}

A positive correlation was detected between $\mathrm{Cho} / \mathrm{Cr}$ levels in the right dorsolateral prefrontal cortex and clinical pain $(r=0.603, p=$ 0.02 ). Evoked pain threshold did exhibit correlations with NAA/ Cho ratios in the left insula $(\mathrm{r}=-0.686, \mathrm{p}<0.01)$ and left basal ganglia $(r=-0.779, r<0.005)$. A negative correlation was seen between the FA values in the right thalamus and clinical pain $(r=$ $-0.50, \mathrm{p}=0.049$ ).

CONCLUSIONS

There are significant correlations between clinical symptoms and metabolite ratios as well as DTI parameters in a number of regions implicated in pain processing in the setting of FM. Further investigation of these findings in the setting of pain stimulation and response to treatment could yield important clues regarding the pathogenesis and management of this disease.

\section{PO4:11}

\section{APPLICATION 3.0-TESLA MAGNETIC RESONANCE IN NEUROLOGICAL DISEASES}

T. Moreno, J. Rodriguez, J. Onofre

Hospital Christus Muguerza Alta especialidad, Monterrey, Mexico

\section{OBJECTIVE}

Describe the utility of 3.0-Tesla Magnetic Resonance (3 T-MR) in neurological pathologies. Materials and methods: We used 3 TMR using fiber tracking with diffusion tensor imaging (DTI), BOLD (Blood Oxygen Level Dependent), Diffusion, Spectroscopy and cerebral perfusion. Results: We obtain 3D tracts reconstructions of the corticospinal via in separate tracts, manual motor and imaging motor activation, diffusion, spectroscopy and highresolution imaging in tumours, inflammatory and cerebrovascular disease. Conclusion: Imaging at $3 \mathrm{~T}-\mathrm{MR}$ provides the highest definition and sensitivity in neurological diseases. The $3 \mathrm{~T}$ morphological and functional imaging can become the standard for high-resolution investigation of brain disease.

\section{P04:12}

\section{USAGE OF MRS AND DWI IN THE DIFFERENTAL DIAGNOSIS OF MASS LIKE LESION IN THE BRAIN AS CORRELATE TO THE PATHOLOGY}

\section{P. Metarugcheep, K. Srusubat, S. Putticharoenrat}

Department of Neuroradiology Prasat Neurological Institute, BKK, Thailand

\section{PURPOSE}

MRS and DWI have been used for the differentiation and grading of the tumor for more than 10 yrs. We attempted to study the benifit of these two techniques in MR imaging as correlte to the pathology

Methods:

80 patients with mass like lesions diagnosed by conventional MRI and pathological or clinical proved (13 low grade astrocytomas, 10 high grade astrocytoma, 9 metasataasis, 5 lymphoma, 3 germinomas, 1 medulloblastoma, 1 hemangioblastoma and 1 neuroblastoma as well as 18 menigiomas,5 schawannomas, and 14 non-tumor) and 20 normal controls were prospectively evaluated with conventional MRI,MRS and DWI.

RESULT

MRS shows benifit in differentiate tumor from non-tumor $(\mathrm{p}<0.5)$, benign form malignant $(\mathrm{p}<0.5)$ and intraaxial from extraaxial.. $\mathrm{Cho} / \mathrm{Cr}>1.3$ is th e key value from differentiate tumor from nontumor and the increase frequency of lactate or lipid for differentiate malignant from benign tumor.The Cho mono paek may be characteristic of extraaxial tummor.Alanin is seen in only meningioma. DWI shows no efficacy for grading tumor or differentiate tumor from non-tumor.Only restricted diffusion are noted in two brain abscesses.

CONCLUSION

In our study,MRS shows benifit in differentitate tumor from nontumor,benign form malignant and intra axial from extraaxial tumor.The $\mathrm{Cho} / \mathrm{Cr}>1.3$ is the cut point for differentiate tumor from non-tumor.The increase frequency of lactate or lipid help to differentiate malignant form benign. Cho mono peak may be feature of extraaxial tumor. DWI shows no efficacy in the differentiate or grading of the tumor.Only restricted diffusion is found in two brain abscesses.

\section{PO4:13}

\section{COMPARISON OF METABOLIC RATIOS OBTAINED WITH 2D-CSI MR SPECTROSCOPY PERFORMED ON 1.5T AND 3T IN HEALTHY CONTROLS.}

G. Beyer, Y. Pang, S. Rohrer, F. Londy, T. Chenevert, P. Sundgren Department of Radiology, University of Michigan, Ann Arbor, USA 


\section{PURPOSE}

High-field MR scanners are becoming more available both in clinical practice and in the research environment. One of their benefits is in the advanced techniques like MR spectroscopy. Previous experimental and in-vivo studies have demonstrated differential T1-relaxation effects on brain metabolites but few studies have compared metabolic ratios obtained on MR units of different field-strength. The purpose of this study is to evaluate for possible significant differences in measured metabolic ratios in healthy subjects examined on both 1.5 and $3 \mathrm{~T}$.

\section{MATERIAL AND METHODS}

Eleven healthy subjects were examined on both a $1.5 \mathrm{~T}$ scanner (LX EchoSpeed, GE Medical Systems) and a 3 T scanner (Philips Achieva MRI system). Two-D CSI MR spectroscopy was performed on both scanners using same TE and TR values. The $\mathrm{NAA} / \mathrm{Cr}, \mathrm{Cho} / \mathrm{Cr}$, Cho/NAA and NAA/Cho ratios were calculated. $\mathrm{P}$-value of $<0.05$ was set for statistical significance (paired t-test). The metabolic spectra on the $1.5 \mathrm{~T}$ scanner were analyzed using the individual vendor software (Functool 2, GE) while the metabolic spectra obtained on the $3 \mathrm{~T}$ were analyzed using an in-house fitting model based on IDL routines.

RESULTS

There were no significant differences in $\mathrm{Cho} / \mathrm{Cr}$ ratios between scanners. NAA/Cr and NAA/Cho ratios were significantly higher $(\mathrm{p}>0.05)$, and Cho/NAA ratios were significantly lower $(\mathrm{p}>0.05)$ on $3 \mathrm{~T}$ compared to $1.5 \mathrm{~T}$ in evaluated areas.

\section{DISCUSSION}

Ideally, metabolite ratios should not have field strength dependence in clinical practice. Nevertheless, there are known differential $\mathrm{T} 1$ and $\mathrm{T} 2$ relaxation effects on the metabolic compounds. The present study demonstrates that other factors beside relaxation play a role.

\section{PO4:14}

WHOLE BRAIN REAL TIME FMRI OF LANGUAGE AND MOTORIC HAND AREAS WITH PROSPECTIVE ACQUISITION CORRECTION FOR MOVEMENTS (PACE)

S. Langner, C. Zimmermann, M. Kirsch, M. Domin, N. Hosten Institute for Diagnostic Radiology and Neuroradiology, ErnstMoritz-Arndt University, Greifswald, Germany

\section{PURPOSE}

Mapping of language and hand-motoric oriented brain areas have become an important clinical application of fMRI for neurology and neurosurgery. We assessed feasibility of online presentation of images during acquisition with prospective acquisition correction for movements (PACE).

\section{METHODS AND MATERIALS}

20 normal subjects and 11 patients with temporal lobe epilepsy or intracerebral tumour were examined at $1.5 \mathrm{~T}$ (Symphony Siemens). An 8-channel head coil was used for BOLD imaging with an EPI GRE sequence (TE $50 \mathrm{msec}$, TR $3000 \mathrm{msec}, 33$ slices, $64 \times 64$ matrix, $3 \mathrm{~mm}$ slice thickness, FOV 192). A semantic decision and a letter matching task alternated every $24 \mathrm{~s}$. The motoric-paradigm was an alternation finger-tapping task. A z-score of 4 was chosen as a cut-off for the t-test evaluation. The evaluation of language lateralization for each language area and each hemisphere occurred with a lateralisation index.

\section{RESULTS}

Table time was under $20 \mathrm{~min}$ for language and $13 \mathrm{~min}$ for motoric examination. In 19/20 subjects language lateralization and in 20/ 20 subjects the motoric hand area could be visualized. For all patients the investigation was diagnostic according to the paradigm. Using a speech paradigm it took 60 after the EPI BOLD sequence was started until the first statistical approximation appeared as an image on the monitor. Functional information was projected on the first EPI images themselves.

In all of 17 right-handed subjects speech areas were mainly left hemispheric (Broca region: $p=0,000$; Wernicke region: $p=0,003$ ). The two left handed subjects showed a different activation: one was left and one mainly right hemispheric.

\section{CONCLUSION}

Real time fMRI is a feasible technique in clinical routine for mapping language and motoric hand areas and gives a reliable feedback of the activation after $60 \mathrm{~s}$ of paradigm runtime.

\section{PO4:15}

\section{FMRI USING A SEMANTIC DECISION PARADIGM: INFLUENCE OF THE ANALYSIS THRESHOLD}

\section{S. Langner, C. Zimmermann, M. Kirsch, N. Hosten}

Institute for Diagnostic Radiology and Neuroradiology, Ernst-Moritz-Arndt University, Greifswald, Germany

\section{PURPOSE}

Functional magnetic resonance imaging (fMRI) can be used to evaluate cortical activity of speech-associated brain areas in a taskspecific manner. The sensitivity and specificity of fMRI results depend on many factors including the imaging technique used and selection of an appropriate paradigm. The aim of the study was to systematically evaluate the influence of the analysis threshold on the size and location of the activated areas.

\section{METHODS}

Eighteen healthy right-handed subjects were examined by fMRI using a semantic decision paradigm in a block design. The active condition and a control condition alternated every 24 seconds. All examinations were performed on a 1.5 T MRI scanner (Magnetom Symphony, SIEMENS). Bold-Imaging were performed using an EPI GRE-Sequence ( 33 slices, $3 \mathrm{~mm}$ slice thickness, TE $50 \mathrm{msec}$, TR $3000 \mathrm{~ms}$, matrix $64 \times 64)$. Real-time data analysis was performed using BOLD analysis software provided by the manufacturer. Postprocessing was repeated using analysis thresholds from 2 to 7 incremented in steps of 0.5 . Wilcoxon's test was chosen to evaluate the statistical significance of language lateralization. 


\section{RESULTS}

Language lateralization was visualized in 17/18 subjects. Employing z-scores $>5$ only left hemispheric speech-associated areas were detected. Using a $\mathrm{Z}$-score $<3$ most subjects showed additional, though weaker, right hemispheric activation. And the size of the left hemispheric areas increased.

\section{CONCLUSION}

Functional MRI allows analysis of speech lateralization noninvasively. Choice of the significance level determines the exact size of the speech-related areas identified and whether speechrelated areas are detected in the nondominant hemisphere or not. The threshold cannot be standardized. Instead, fMRI should be performed with several postprocessing runs with different thresholds depending on clinical and experimental settings.

\section{PO4:16}

\section{SYNESTHETIC DISPLAY OF PROTON MAGNETIC RESONANCE SPECTROSCOPIC DATA TO ENHANCE INTEPRETATION}

M. Kuhn ${ }^{1}$, J. Espinosa ${ }^{1}$, A. Kuhn ${ }^{2}$

${ }^{1}$ Southern Illinois University School of Medicine, Springfield, USA; ${ }^{2}$ University of Noth Carolina, Chapel Hill, USA

\section{PURPOSE}

Synesthesia is a perceptual phenomenon which, in one of its manifestations, numbers, letters or shapes are perceived as certain colors; this is termed "grapheme". The use of graphemes has been shown to increase visual perception and comprehension. We performed a study in which various normal and abnormal peaks of proton magnetic resonance spectroscopy of the brain were assigned different colors in order to evaluate improvements in perception by radiology trainees, trainees in allied fields and board certified radiologists.

\section{METHODS}

Two board certified radiologists, six diagnostic radiology residents and four neurology residents in all levels of training were presented with proton magnetic resonance spectra on 31 patients with brain neoplasms (14), cerebral infarcts (12), brain radiation necrosis (3) and metabolic diseases (2). Based on a pilot study, the choline peak was assigned as black, NAA was assigned red, lactate was assigned blue, creatine was assigned green and glutamate-glutamine were assigned yellow. After an initial training period, the subjects were shown examples of standard monochrome spectra and grapheme based colorized spectra and were asked to make a diagnosis as quickly as possible.

RESULTS

Grapheme based spectral diagnoses were achieved with $96.8 \%$ accuracy in an average of 2.8 seconds. Standard monochrome spectral diagnsoes achieved $90.3 \%$ accuracy with an average time of diagnosis of 6.1 seconds. The difference in accuracy and time of diagnosis were significant to $\mathrm{p}<0.001$.

\section{CONCLUSIONS}

Our experience suggests that the use a grapheme based display of proton magnetic resonance spectroscopic data will improve accuracy and efficiency of proton magnetic spectroscopy diagnosis.

\section{PO4:17}

\section{VALIDATION OF TECHNIQUES FOR MEASURING HIPPOCAMPUS VOLUME: COMPARISON OF MANUAL AND AUTOMATIC METHODS}

\section{S. Kim, K. Lee, W. Tae, E. Nam, K. Kim}

Neuroscience Research Institute, Kangwon National University College, of Medicine Chuncheon, South Korea

\section{PURPOSE}

With the increased use of automated volumetry methods using cutting-edge techniques in brain research, the validation of each program is crucial. We examined the validity of two currently used automated volumetry methods (surface-based parcellation using FreeSurfer and individual atlas-based volumetry using IBASPM) for estimating the hippocampus volume in comparison with manual volumetry as the gold standard.

\section{METHODS}

3-Dimensional (3D) T1-weighted volume magnetic resonance (MR) data were obtained using the Coronal turbo field echo (TFE) technique in eight healthy female volunteers and seven patients with major depressive disorder (age range 35 to 53 years, mean 45 years). The volumes of the right and left hippocampus were measured in each using three different methods: manual drawing, surface-based parcellation using FreeSurfer, and individual atlas-based volumetry using IBASPM. The results were compared statistically using Pearson's correlation test.

RESULTS

The mean volume of the right hippocampus was $2563.98 \mathrm{~mL}(\mathrm{SD}=$ 413.54, min-max: 1452-3133 mL) determined manually, 3978.53 ( $S D=249.27$, min-max: 3544-4372) using FreeSurfer, and 2893.40 (SD=523.05, min-max: 1568-3463) using IBASPM. The respective mean volumes of the left hippocampus were $2547.08 \mathrm{~mL}(\mathrm{SD}=$ 350.74, min-max: 1437-2904), 3722.53 ( $\mathrm{SD}=301.75$, min-max: 3091-4248), and 3342.20 ( $\mathrm{SD}=498.02$, min-max: 1973-3863). Both hippocampus volumes determined using IBASPM were correlated with the manual results (RT: $r=0.697, p=0.004$, LT: $r=$ $0.628, p=0.012)$, while those using FreeSurfer were not (RT: $r=$ $0.420, \mathrm{p}=0.119, \mathrm{LT}: \mathrm{r}=0.334, \mathrm{p}=0.223$ ).

\section{CONCLUSIONS}

Compared with manual volumetry, automatic volumetry techniques tend to overestimate the hippocampus volume and result in small or large discrepancies. Manual drawing should still be used to measure the hippocampus volume despite the convenience of automated methods. 


\section{PO4:18}

\section{COMPARISON OF FRACTIONAL ANISOTROPY \\ OF THE CORPUS CALLOSUM: MID-SAGITTAL VS. AXIAL IMAGING}

E. Kim ${ }^{1}$, J. Lee ${ }^{1}$, H. Park ${ }^{1}$, D. Kim ${ }^{2}$

${ }^{1}$ Department of Radiology and Research Institute of Radiological Sciences, Yonsei University College of Medicine, Seoul, South Korea; ${ }^{2}$ School of Electrical and Electronic Engineering, Yonsei University, Seoul, South Korea

\section{PURPOSE}

DTI is usually obtained with a relatively thick slice in axial plane, causing a partial volume effect. We hypothesized that FA of the CC can be more accurately measured using mid-sagittal DTI than axial DTI. The purpose of this study was to compare FA values of the CC of healthy subjects who underwent both axial and mid-sagittal DTI. METHODS

Fourteen healthy volunteers (each of 7 male and female; mean age, 29.1 years) underwent MRI at 3.0 T. DTI with 45 directions was performed in both mid-sagittal and axial planes. One 5-mm midsagittal image and 20 2-mm axial images were obtained for the CC. The five ROIs were carefully drawn along the border of the corpus callosum on each sagittal FA map (Figure). The FA values obtained from each region were compared between two sagittal maps. RESULTS

The mean FA values of the regions I-V in the mid-sagittal imaging were $0.77 \pm 0.04,0.72 \pm 0.04,0.76 \pm 0.03,0.77 \pm 0.03$, and $0.80 \pm$ 0.03 , respectively. The mean FA values of the regions $\mathrm{I}-\mathrm{V}$ in the mid-sagittal image from axial imaging were $0.74 \pm 0.04,0.67 \pm$ $0.03,0.70 \pm 0.05,0.69 \pm 0.06$, and $0.78 \pm 0.03$, respectively. The FA values of all regions except for the region $\mathrm{V}$ significantly increased in the mid-sagittal imaging. The FA values in the region IV were significantly underestimated in the mid-sagittal image from axial imaging, compared with those in the regions $\mathrm{I}$ and $\mathrm{V}$ $(\mathrm{p}=.037$ and $\mathrm{p}=.001$, respectively; post hoc test).

\section{CONCLUSION}

FA values of the $\mathrm{CC}$ were significantly higher with mid-sagittal DTI than with axial DTI in regions I-IV, particularly in the region IV. Mid-sagittal DTI may provide more accurate FA values of the CC than axial DTI, and may be more desirable for comparative studies between patients and healthy subjects.

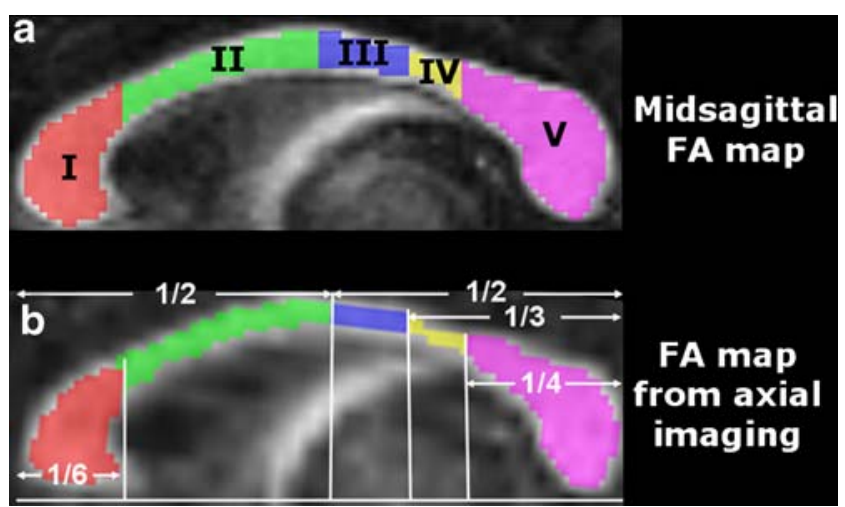

PO4:19

MR IMAGING IN PATIENTS WITH SUSPECTED DIFFUSE AXONAL INJURY (DAI): DIFFUSION WEIGHTED IMAGING $\&$ T2* GRE SEQUENCE

P. Ellinas ${ }^{1}$, K. Bakopoulos ${ }^{2}$, V. Katsaros ${ }^{3}$, S. Lyra ${ }^{1}$, A. Papatheodorou ${ }^{1}$, E. Samaras ${ }^{2}$, V. Varsos ${ }^{2}$, N. Batakis ${ }^{1}$

${ }^{1}$ Radiology department, Hellenic Red Cross Hospital, Athens, Greece; ${ }^{2}$ Neurosurgery department, Hellenic Red Cross Hospital, Athens, Greece; ${ }^{3}$ Neuroradiology Department, IKA Oncology Hospital, Athens, Greece

\section{PURPOSE}

The role of MRI in the evaluation of diffuse axonal injury of the brain is well established. DAI is the result of severe closed or open head injury and is usually located in corpus callosum, brainstem, basal ganglia and grey/white mater interface. The most of DAI are non-haemorrhagic and it is considered that only a small percentage of them are visible with CT or MR examination. We evaluate the contribution of diffusion weighted imaging \& T2* GRE sequences in the detection of DAI.

\section{METHODS}

15 patients with closed brain injury referred for MRI examination throughout the last 6 months. All the patients had symptoms suspicious for DAI. The examination protocol, included: T2weighted TSE, FLAIR, T1-weighted SE, DWI and T2* sequences. RESULTS

All the 15 patients proved to have DAI. The haemorrhagic lesions in all patients were depicted at $\mathrm{T} 2 *$ sequence. In 11 patients (examined within 72 hours after the accident) the DWI depicted all the lesions that were detected with T2* sequence. In 3 patients the DW imaging revealed additional lesions without haemorrhagic component. In 4 patients (examined about 10 days after the accident), the DWI was negative.

\section{CONCLUSION}

A fast examination protocol, using diffusion weighted imaging in combination with T2* GRE sequence, within the first 24 hours, is essential for the diagnosis and prognosis of patients with closed head injury.

\section{PO4:20}

\section{COMPLICATED ARACHNOID CYST WITHOUT HISTORY OF HEAD INJURY OR ANEURYSM RUPTURE}

V. Katsaros $^{1}$, T. Flaskas ${ }^{2}$, G. Strantjalis ${ }^{2}$, E. Boviatsis ${ }^{2}$,

$\overline{\text { S. Gatzonis }}^{2}$, C. Chrysikopoulos ${ }^{3}$, D. Sakas ${ }^{2}$

${ }^{1}$ IKA Oncology Hospital, Department of CT and MRI, Athens, Greece; ${ }^{2}$ Evangelismos Hospital, Department of Neurosurgery, University of Athens, Athens, Greece; ${ }^{3}$ Eurodiagnosi Diagnostic Center, Corfu, Greece

\section{STUDY DESIGN}

A case report BACKGROUND DATA: Arachnoid cysts are intraarachnoid cerebrospinal fluid collections that are usually asymp- 
tomatic. However, they may become acutely symptomatic due to enlargement of the cyst or the presence of haemorrhage.

\section{MATERIALS AND METHODS}

We report a case of a woman presenting with history of headache, deteriorating the last weeks, but without nausea, vision problems or any other symptoms. There was no history of trauma. Diffusion-weighted MRI clearly demonstrated a large right anterior cranial fossa arachnoid cyst with associated subacute intracystic but no subdural haematoma or aneurysm.

\section{RESULTS}

This lesion was isodense compared to brain on CT. Conventional MRI could not distinguish the presence of intracystic haemorrhage. CONCLUSION

We focus on the importance of diffusion-weighted MR imaging in the differentiation of these late subacute/early chronic haemorrhagic collections that may be overlooked with $\mathrm{CT}$ and conventional MRI.

\section{PO4:21}

FAILURE OF DECUSSATION OF IMPORTANT PATHWAYS DEMONSTRATED BY MR TRACTOGRAPHY IN JOUBERT SYNDROME

T. Huisman ${ }^{1}$, A. Poretti ${ }^{2}$, T. Loenneker ${ }^{1}$, E. Valente $^{3}$, F. Brancati ${ }^{3}$, K. Ilyasov $^{1}$, E. Boltshauser ${ }^{2}$

${ }^{1}$ Department of Diagnostic Imaging, University Cildren's Hospital Zurich, Zurich, Switzerland; ${ }^{2}$ Department of Pediatric Neurology, University Cildren's Hospital Zurich, Zurich, Switzerland;

${ }^{3}$ Neurogenetics, CSS Mendel Institute, Rome, Italy

\section{INTRODUCTION}

Joubert syndrome (JS) is an autosomal recessively inherited hindbrain malformation syndrome with marked genetic heterogeneity. Neuropathological findings and preliminary imaging studies showed absence of pyramidal tract and superior cerebellar peduncles decussation. AHI1, the first gene to be associated with JS, is mostly expressed in neurons that give rise to crossing axons of these tracts. With MRI, diffusion tensor imaging (DTI) and tractography, we studied the course of these fiber tracts in five patients with JS.

\section{METHODS AND PATIENTS}

MRI was performed using a 3.0 Tesla MRI-Unit. Conventional imaging was followed by DTI . Diffusion encoding was performed along 20 directions evenly distributed over a sphere with an effective b-factor of $1000 \mathrm{~s} / \mathrm{mm} 2$. Images were corrected for eddy current induced geometrical distortions. Isotropic diffusion weighted images (DWI), apparent diffusion coefficient (ADC) and fractional anisotropy (FA) maps were calculated including tractography. Imaging showed in all cases vermis agenesis and the molar tooth sign. One patient had a mutation in the AHI1 gene on chromosome 6 and two patients a mutation in the CEP 290 gene on chromosome 12. The mutation of the other patients is not yet known.

\section{RESULTS}

In all patients the fibers of the superior cerebellar peduncles projected to the ipsilateral red nuclei and thalami directly without decussating in the mesencephalon. In $3 / 5$ patients the corticospinal tract failed to cross in the medulla oblongata. In 2 patients. the corticospinal tract could not be displayed satisfactorily within the lower brainstem due to magnetic field inhomogeneity along the skull base.

\section{CONCLUSION}

In JS corticospinal tract and the fibers from the superior cerebellar peduncles do not cross irrespective of the underlying mutation. The interaction of the various gene products regarding tract projections and brainstem anatomy is not yet known. Tractography furthers our understanding of the modified or aberrant fibers in JS.

\section{PO4:22}

\section{MULTIMODAL IMAGING IN RASMUSSEN ENCEPHALITIS}

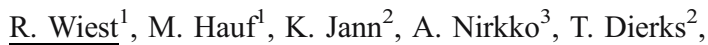
A. Federspiel $^{2}$

${ }^{1}$ Institute of diagnostic and interventional Neuroradiology, University of Berne, Berne, Switzerland; ${ }^{2}$ Department of Psychiatric Neurophysiology, University of Berne, Berne, Switzerland; ${ }^{3}$ Department of Neurology, University of Berne, Berne, Switzerland

\section{PURPOSE}

Rasmussen's encephalitis (RE) is a rare, inflammatory disorder occurring in children, which usually affects one hemisphere of the brain. Most patients with Rasmussen's encephalitis suffer from frequent seizures and progressive atrophy of the affected hemisphere. We report results of recent imaging techniques identifying the irritative area and the extension of RE.

\section{METHODS}

A five years old girl, which suffers from non-convulsive status epilepticus (NCSE) during the early course of RE, was examined by resting state fMRI analyzed by cortex-based spatial independent fast component analysis (ICA), resting state CBF measures with arterial spin labeling (ASL), diffusion tensor imaging (DTI) and measurements of the cortical thickness. The MRI data were matched with EEG source location data. The analysis of the multimodal dataset was performed with MATLAB (Perfusion) and with BrainVoyager QX 1.7. (fMRI-BOLD measure and cortical thickness analysis). All DTI images were analyzed using DTIStudio and EEG source analysis with LORETA.

\section{RESULTS}

The irritative area was confirmed by ICA analysis of the resting state fMRI. One ICA factor coded for an independent neuronal activity in the right temporal lobe, fitting the increased $\mathrm{CBF}$ as measured by ASL and the spike localisation on the EEG surface map. DTI and cortex showed reduction of the white matter tracts in the affected region with corresponding reduction of grey matter thickness, which helped to delineate the brain areas affected by RE.

\section{CONCLUSION}

Multimodal imaging techniques can be additionally applied to a routine MRI protocol and may provide complementary and convergent information abut the extension of the RE and seizure origins. 


\section{PO4:23}

\section{UNCINATE AND LONGITUDINAL INFERIOR BUNDLES IN TEMPORAL LOBE EPILEPSY : PRELIMINARY STUDY WITH DIFFUSION TENSOR IMAGING}

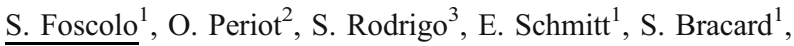
M. Braun ${ }^{1}$

${ }^{1} \mathrm{CHU}$ Nancy, Nancy, France; ${ }^{2} \mathrm{CHU}$ Bordeaux, Bordeaux, France $;^{3}$ CHU Saint Anne, Paris, France

Temporal lobe epilepsy (TLE) is the most common partial epilepsy syndrom and unilateral mesial temporal sclerosis (MTS) one of the principal causes. This preliminary study proposes to determine if a possible axonal degeneration in two white matter tracts, uncinate and longitudinal inferior bundles, close to limbic tracts, can be demonstrated using diffusion tensor imaging (DTI) and tractography in a group of epilepsy patients with MTS.

Two groups, nine patients with unilateral MTS and ten control subjects, are evaluated. Images are acquired using a GE 1.5 T MRI scanner ( 35 directions, $3 \mathrm{~mm}$ ). The Anatomist $(\mathrm{C}$ and BrainVISA $\mathrm{C}$ softwares are used for post processing with a reproducible technique of bundles tracing. Diffusion measurements (ADC, mean bulk mobility of water, and FA, index of fractional anisotropy) are obtained from all voxels forming the three-dimensional structures then analyzed with nonparametric tests.

No significant difference in ADC and FA between right/left is observed in the control group.

On the other hand, a modification of diffusion parameters in the two bundles in the patient group is observed with, notably, a bilateral reduction in FA values and a bilateral increase in the ADC values. This preliminary study, by suggesting bilateral abnormalities in the structure of the uncinate and longitudinal inferior bundles in TLE, needs further investigations to confirm these results in a large group of patients, and to better understand the neuronal physiopathological mechanisms.

\section{PO4:24}

\section{CT-PERFUSION: ASSESSMENT OF NORMAL BRAIN HEMODYNAMIC.}

L. Fadeeva, I. Pronin, N. Zakharova, M. Dolgushin, V. Kornienko Burdenko Neurosurgery Institute, Moscow, Russia

\section{MATERIALS AND METHODS}

This study included 6 volunteers (4 M, 2 F, aged 21-36 years: mean 30 years) without some neurosurgical pathology or neurologic signs and the group of 13 patients with brain tumors (6-glioblastomas and 6- metastasis of cancer, $8 \mathrm{M}, 5 \mathrm{~F}$, aged 2642). All of them underwent $\mathrm{CT}$ perfusion imaging. $\mathrm{CBV}, \mathrm{CBF}$ and MTT values were measured in the regions of WM (frontal, parietal:ROI 100 mm2), GM (putamen, thalamus:ROI $90 \mathrm{~mm} 2$, frontal/parietal core: ROI $25 \mathrm{~mm} 2$ ) and large opercular ROI (area 1400 mm2) in the both hemispheres for volunteers. For the patients with brain tumor measurements were made in similar ROI on the contra-lateral to tumor hemisphere.

RESULTS

$\mathrm{CBV}, \mathrm{CBF}$, MTT values, measured in various zones of WM, show no significant difference both for hemispheres and for locations, and consisted $0,81+/-0,19 \mathrm{ml} / 100 \mathrm{~g}, 10,32+/-1,59 \mathrm{ml} / 100 \mathrm{~g} / \mathrm{min}$, $5,2+/-0,5 \mathrm{sec}$, respectfully. There wasn't significant difference between $\mathrm{CBV}, \mathrm{CBF}$ and MTT values measured in GM ROIs in both hemispheres, but values varied in different anatomic locations. Minimal CBV, CBF values were measured in thalamus: $1.37+/-0.16 \mathrm{ml} / 100 \mathrm{~g}$ and $28.40+/-2.83 \mathrm{ml} / 100 \mathrm{~g} / \mathrm{min}$. The same values, measured in putamen, small WM ROIs and large opercularl ROIs, were statistically higher $(p<0.01)$. CBV, CBF, MTT values in WM ROIs were in the limits of confidence intervals for volunteers on contra-lateral to tumor hemisphere. $\mathrm{CBV}$ values in GM were found to be higher in patients with metastasis than in pts with glioblastomas and in volunteers, but MTT values were longer in patients with glioblastomas $(\mathrm{p}<0.05)$. CONCLUSION

The results, obtained in this study may be taken as reference points for future quantitative assessment of brain perfusion.

\section{PO4:25}

\section{CEREBRAL ABSCESSES AND NECROTIC CEREBRAL TUMORS: DIFFERENTIAL DIAGNOSIS BY PERFUSION-WEIGHTED MAGNETIC RESONANCE IMAGING.}

C. Muccio $^{1}$, G. Esposito ${ }^{1}$, A. Bartolini ${ }^{1}$, A. Cerase ${ }^{2}$

${ }^{1}$ UO Neuroradiologia, Azienda Ospedaliera G. Rummo, Benevento, Italy; ${ }^{2}$ UOC Neuroradiologia, Policlinico Santa Maria alle Scotte, Siena, Italy

The purpose of this study was to evaluate the usefulness of Perfusion-weighted imaging (PWI) in the differential diagnosis of cerebral lesions presenting with "ring- enhancement" including abscesses, high-grade gliomas, and metastases.

Nine cerebral abscesses (5 piogenic, 4 from Toxoplasma Gondii), 11 glioblastomas, and 5 cerebral metastases in 20 patients underwent gadolinium-enhanced Magnetic Resonance imaging, Diffusion-weighted imaging including calculation of average value of apparent diffusion coefficient (DWI-ADC) of the lesions core, and PWI. At PWI, the medium value of the maximum relative cerebral blood volume (rCBV) was calculated in the gadolinium-enhancing peripheral solid areas and compared with that of the contralateral normal-appearing white matter, as ratio $=\mathrm{rCBV}$ (lesion) $/ \mathrm{rCBV}$ (contralateral normal-appearing white matter).

DWI-ADC achieved the differential diagnosis in all cases, except for the 4 abscesses from Toxoplasma Gondii. At PWI, the medium ratio of the $\mathrm{rCBV}$ of the capsular portion was $0.72+/-0.08$ (range: $0.6-0.82$ ) in the piogenic abscesses, $0.84+/-0.07$ (range: $0.75-$ 0.91 ) in the abscesses from Toxoplasma Gondii, $4.45+/-1.5$ (range: $2.9-8$ ) in the high-grade gliomas, and $3.58+/-0.68$ (range: $3.28-4.27$ ) in the metastases.

In conclusion, PWI seems to be useful in the differential diagnosis of cerebral lesions presenting with "ring-enhancement". High 
rCBV values in the peripheral areas should indicate the possibility of a necrotic tumor, while low values tend to indicate an abscess.

\section{PO4:26}

\section{FEASIBILITY OF WHOLE BODY DIFFUSION WEIGHTED IMAGING IN USING NCPMG-SSFSE TECHNIQUE}

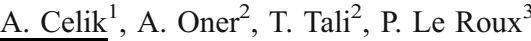

${ }^{1}$ GE Healthcare, Antalya, TURKEY; ${ }^{2}$ Gazi University Department of Radiology, Ankara, Turkey; ${ }^{3}$ GE Healthcare ASL department, Buc, France

\section{PURPOSE}

Using a new non-Carr-Purcell-Meiboom-Gill (non-CPMG) singleshot fast spin-echo (ssFSE) tecnique, to investigate the feasibility of a true whole body DWI (WB-DWI) and compared it to standard EPI based DWI technique (1).

\section{METHODS}

An 62 years old male patient with a multiple metastatic tumors in L4, T8, right lung and left femural bone was scanned using Coronal nCPMG ssFSE DWI sequence, Axial EPI DWI using a b-value of $600 \mathrm{~s} / \mathrm{mm} 2$.on a $1.5 \mathrm{~T}$ scanner (GE Healthcare, Milwaukee, WI), and image sets were evaluated for susceptibility artifacts, tumor delineation, overall image quality, contrast and signal to noise ratio (CNR, SNR).

RESULTS

EPI-DWI images showed typical susceptibility artifacts, compared to artifact free nCPMG-ssFSE DWI images. Axial EPI-DWI images are found to be less affected of susceptibility artifacts, but suffered from lower in-plane resolution compared to the nCPMGssFSE DWI. Even though both technique delineated all the tumors, the nCPMG-ssFSE DWI technique provided increased inplane resolution, SNR, CNR and overall image quality, comparable to $\mathrm{T} 2$-weighted images, without any time penalty.
Figure.1: Whole Body DWI and T2 images: Axial EPI DWI images reformatted and MIPed to coronal plane $(a, b)$, coronal nCPMG dDWI images of corresponding slices (c.d) and SSFSE T2 weighted image of whole body (e .f).

\section{CONCLUSION}

This paper demonstrates the feasibility of a new true whole body DWI technique in a reasonable acqusuiton time with no postprocessing required. This technique improves the in-plane resolution, provides higher SNR, reduces susceptibility artifacts and could offer a great alternative to PET imaging.

REFERENCES

[1] Le Roux P, JMR 2003;155:278-292.

\section{P04:27}

\section{VALIDATION OF MR DIFFUSION WEIGHTED IMAGING USING NCPMG-SSFSE SEQUENCE}

\section{A. Celik ${ }^{1}$, A. Oner ${ }^{2}$, T. Tali ${ }^{2}$, P. Le Roux ${ }^{3}$}

${ }^{1}$ GE healthcare, Antalya, Turkey; ${ }^{2}$ Gazi University Department of Radiology, Ankara, Turkey; ${ }^{3}$ GE Healthcare ASL Department, Buc, France

\section{PURPOSE}

The recently developed Non-Carr-Purcell-Meiboom-Gill sýngleshot fast spin-echo (nCPMG-ssFSE) sequence is a recent alternative to Echo Planar Imaging (EPI) for diffusion weighted imaging (DWI) (2). The purpose of this study is to investigate the accuracy of Apparent Diffusion Coefficient (ADC) values measured with nCPMG-ssFSE by comparing to those obtained with EPI DWI in a test phantom and in the well studied organ : brain. METHODS

8 females and 4 male patients were enrolled in this study. Phantom measurements were performed on a $\mathrm{NaCl}$ phantom using both DWI imaging techniques. ADC values were measured over

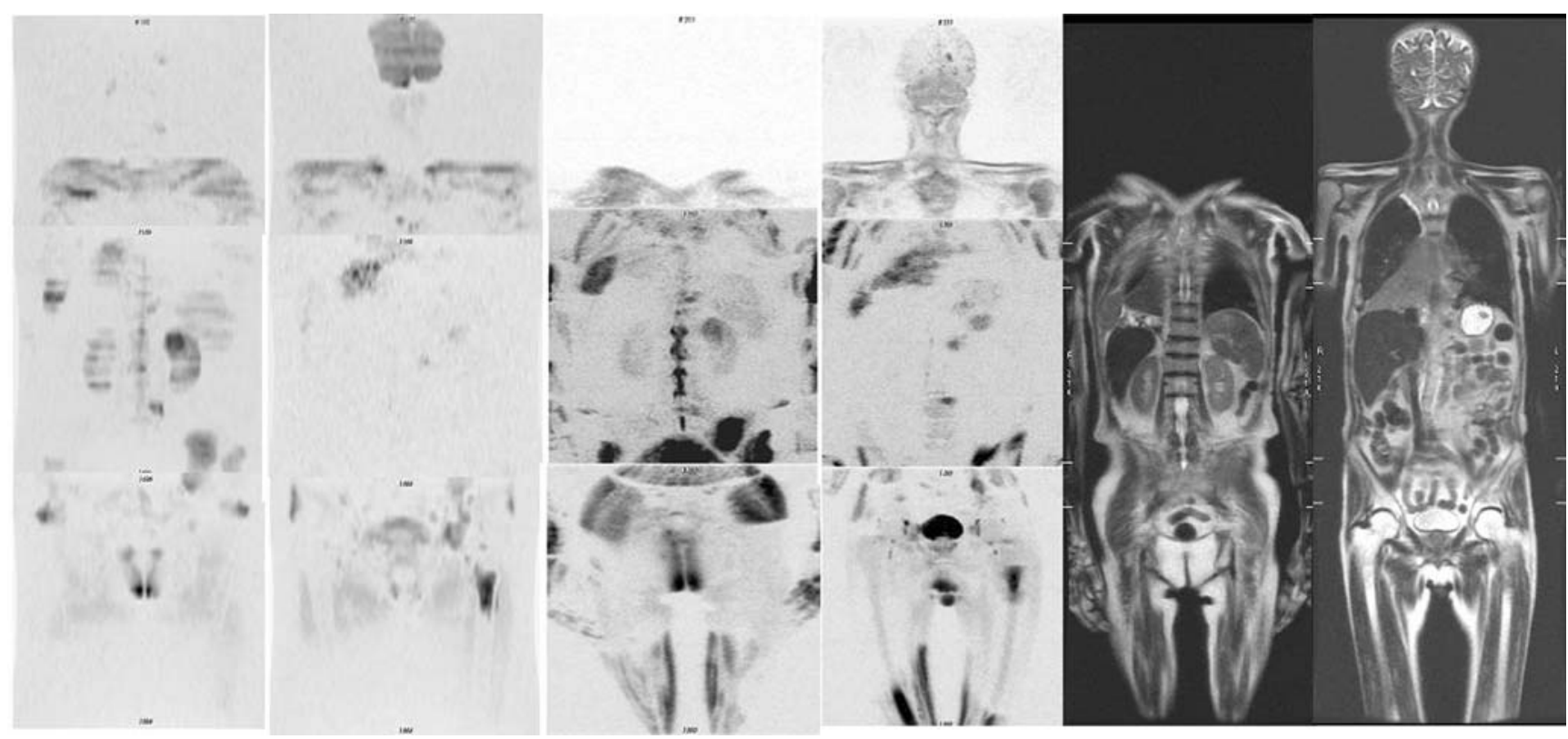


normal appearing white Matter (NAWM) and normal appearing Gray Matter (NAGM) in 6 different locations. Student t-tests were used for statistical analysis.

\section{RESULTS}

Mean ADC values of the phantom using EPI DWI and nCPMG ssFSE were $1598.67 \pm 49.47$ and $2124.13 \pm 33.30 \times 10-6 \mathrm{~mm} 2 / \mathrm{sec}$. The ratio of NCPMG-ssFSE to EPI ADC was $1.33 \pm 0.05$. Mean ADC values of the NAWM and NAGM measured using both techniques were within the range reported in literature (3). However, mean ADC values of the NAWM and NAGM measured using the nCPMG-ssFSE DWI sequence were found to be significantly higher than those obtained with EPI DWI $(\mathrm{p}<$ 0.001). The ratios of NCPMG-ssFSE to EPI ADC for NAWM and NAGM were $1.34 \pm 0.13$ and $1.36 \pm 0.16$, respectively. CONCLUSION

ADC measured with NCPMG-ssFSE method results in higher values than those measured with EPI DWI sequence. This indicates that care must be taken with NCPMG-ssFSE sequence, when normative and pathological values are compared to previously reported results using EPI DWI method.

REF.

[1] Le Roux P, JMR 2003 ;15 [2] Oner AY et. al., ISMRM 2006; 3415, [3] Bastin et. al. MRM 2002, 48:6-14.

\section{PO4:28}

\section{DIFFUSION WEIGHTED MR-IMAGING AND APPARENT DIFFUSION COEFFICIENT IN OSMOTIC MYELINOLYSIS}

\author{
F. Cartes-Zumelzu $^{1}$, L. Kramer ${ }^{2}$, S. Rasoul-Rockenschaub ${ }^{2}$, \\ M. Thurnher ${ }^{1}$ \\ ${ }^{1}$ Medical University Vienna,Dep.of Radiology, Division of \\ Neuroradiology, Vienna, Austria; ${ }^{2}$ Medical University Vienna, \\ Dep.of Internal Medicine, Vienna, Austria
}

\section{PURPOSE}

Osmotic Myelinolysis (OM) is a rare osmolar pathology resulting in demyelination of white brain matter. The disease especially at the beginning is sometimes difficult to diagnose by means of CT and conventional MRI alone.

$\mathrm{OM}$ manifests as a consequence of metabolic derrangements especially in aggressive i.v. fluid correction of hyponatremia. Little information is currently available concerning Diffusion weighted imaging as well as Apparent Diffusion Coefficient measurements in Osmotic Myelinolysis.

Material and Methods: We retrospectively examined the MRI' $\mathrm{s}$ $(1.5 \mathrm{~T})$ of 7 patients suffering of osmotic myelinolysis (OM).

Five of the patients showed a central pontine myelinolysis (CPM) and 2 patients showed an extrapontine (EPM) manifestation. Detailed attention was put on Diffusion weighted Imaging and on ADC Maps. ADC was measured in pathological sites and also in the contralateral normal appearing white matter (NAWM).

RESULTS

All patients showed restricted Diffusion with hyperintense signals in DWI in affected brain areas.
The corresponding ADC Maps showed hypointense signals. ADC Values were significantly lower for affected brain areas in comparison to the contralateral NAWM.

Mean ADC values for affected brain tissue was $0,6 \times 10-3 \mathrm{~mm} 2 / \mathrm{sec}$. Mean ADC for the contralateral NAWM was $0,85 \times 10-3 \mathrm{~mm} 2 / \mathrm{sec}$.

\section{CONCLUSION}

Confirming earlier sparse case observations we have seen significant lower ADC values between affected brain areas in OM and healthy white matter in our patient group. DWI was restricted in all cases.

Existing Literature reports that $\mathrm{ADC}$ values are high in Acute disseminated encephalomyelitis and in Multiple sclerosis.

Accordingly DWI and corresponding ADC values might represent a useful technique to distinguish $\mathrm{OM}$ from other demyelinating brain conditions.

DWI is very sensitive at early time point for ischemia if the same is true for $\mathrm{OM}$ it might represent a useful tool for the early diagnosis of the disease especially as the clinical diagnosis of osmotic myelinolysis can be challenging.

\section{PO4:29}

\section{AN FMRI STUDY OF MUSICOGENIC EPILEPSY: CAN THE EMOTIONAL COMPONENTS OF MUSIC TRIGGER THE SEIZURES?}

D. Cevolani ${ }^{1}$, R. Agati $^{1}$, F. Pittau ${ }^{2}$, F. Bisulli ${ }^{2}$, M. Maffei ${ }^{1}$, S. Musho Ilbeh $^{2}$, I. Naldi ${ }^{2}$, M. Leonardi ${ }^{1}$, P. Tinuper ${ }^{1}$

${ }^{1}$ Neuroradiology O.U. Department Of Neurological Sciences, BOLOGNA, Italy; ${ }^{2}$ Neurology Institute Department

Of Neurological Sciences, Bologna, Italy

Musicogenic epilepsy is a rare form of epilepsy. We describe a 36years-old right-handed man, with right temporal lobe seizures since the age of 27 years. He was an amateur musician and realized that any music with a strong emotional charge was effective in precipitating seizures, even if it was only thought out. All of his seizures were musicogenic and started about 2 min after the beginning of the appropriate music. Anastasia song "Overdue Goodbye" was especially provocative even when he was playing guitar himself. Music without emotional charge (Battisti song "La canzone del sole", or disco music) was ineffective. The patient underwent brain MRI, neuropshycological tests and prolonged video-polygraphic recording.

We performed fMRI to explore the location and extent of the eloquent areas activated during the playing of the two aforesaid songs: neutral music (Battisti song "La canzone del sole") and epileptogenic music (Anastasia song "Overdue Goodbye"). The listening of neutral music showed activated areas in the right temporal lobe in a region corresponding to the acoustic area. The listening of epileptogenic music showed a wider spreading of this area mainly towards the posterior part of the right temporal lobe up to the posterior pole of the right hemisphere, without a concomitant epileptic attack. The thinking of neutral music produced eloquent areas in the frontal pole, bilaterally, but no temporal activation was observed. The thinking of epileptogenic 
music caused a wide spreading of eloquent areas in the left hemisphere, from the very anterior frontal pole to the occipital one. Bilateral frontal activation was also present.

The results are discussed in terms of emotional reaction to music as the stimulus effective in triggering musicogenic seizures.

\section{PO4:30}

\section{PROTON MAGNETIC RESONANCE SPECTROSCOPY FINDINGS IN RASMUSSENS ENCEPHALITIS}

\author{
A. Arslanoglu \\ Van Military Hospital Department of Radiology, Van, Turkey
}

Rasmussens encephalitis is a chronic, progressive epilepsy involving one hemisphere of the brain. Only means of effective seizure control is hemispherectomy. Proton magnetic resonance spectroscopy is a non-invasive technique for the measurement of various metabolites in the brain. We evaluated Proton magnetic resonance spectroscopy findings of a 2-years old boy here. Assessed reductions in N-Acetyl Aspartate are interpreted as being due to neuronal/axonal damage and dysfunction, while increased choline levels may be the result of microglial proliferation.

\section{PO4:31}

\section{FIBER TRACTOGRAPHY AND FA MAP COMBINED METHOD IN DETERMINING PERITUMORAL INFILTRATIONS IN LOW- AND HIGH- GRADE GLIOMAS}

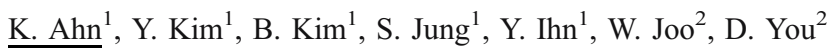
${ }^{1}$ Department of Radiology, Catholic University of Korea, College of Medicine, Seoul, South Korea; ${ }^{2}$ Department of Neurosurgery, Catholic University of Korea, College of Medicine, Seoul, South Korea PURPOSE

Until now a reliable differentiation between tumor infiltration and vasogenic edema is known to be not yet possible on the basis of diffusion tensor imaging (DTI). We want to investigate the usefulness of fraction anisotrophy (FA) map and fiber tractography combined method in determining the peritumoral infiltrations of white matter beyond the tumor edge in various grade gliomas to search for erroneously known as tumor infiltrated region.

METHODS

Ten patients with biopsy or operation-proved glioma ( 2 gliomatosis cerebri, 1 low grade glioma, 1 high grade glioma, and 6 glioblastoma multiformes) underwent conventional MRI and DTI. The tumor mass was defined as the enhancing area in malignant gliomas and as the area of increased T2-signal in low- grade gliomas. FAs were measured on peritumoral hyperintense white matter (WM) seen on T2-weighted MR images and the extent of peritumoral infiltrations were determined. Fiber tractography around the tumor mass was performed, and tumor-induced changes of fiber tracts were evaluated. The changes of the fiber tracts were categorized into: 1) disrupted, 2) reduced in number, 3) displaced, and 4) intact. Two radiologists determined tumor extent and categorized the WM tract changes by consensus, and investigated FA map and fiber tractography mismatch regions to search for erroneously known as tumor infiltrated region. RESULTS

On FA maps of seven high grade gliomas and glioblastoma multiformes, peritumoral low value areas were demonstrated beyond the tumor edge, and these low value areas included lots of major fiber tracts. By using the fiber tractographies we confirmed tumor infiltrations shown as 'disrupted' tract and 'reduced in number' in all seven malignant gliomas. However, among the seven malignant gliomas, five tumors $(7$ regions in total number) showed FA map and fiber tractography mismatches (low FA values, but displaced or intact fiber tracts). In case of low grade glioma, some areas with low FA value were noted, however, these areas showed also mismatches on fiber tractography. In two cases of gliomatosis cerebri, there is no area with low FA value and 'disrupted' or 'reduced in number' on fiber tractography CONCLUSION

In high grade gliomas, peritumoral low FA values were too sensitive to represent tumor infiltrations, and in these cases, fiber tractographies performed around the mass were useful to confirm true infiltrations.

FA map and fiber tractography combined method seems to be very useful in determining and differentiating peritumoral infiltrations and vasogenic edema of white matter beyond the tumor edge in various grade gliomas.

\section{The Aging Brain and Neurodegenerative Disorders}

\section{PO5:1}

\section{STATISTICAL ANALYSIS OF CEREBELLAR BLOOD FLOW IN PATIENTS WITH AUTOSOMAL DOMINANTLY INHERITED PURE CEREBELLAR ATAXIAS.}

\author{
M. Sugawara $^{1}$, K. Obara ${ }^{1}$, M. Kobayashi ${ }^{1}$, H. Ishiguro ${ }^{2}$, \\ I. Toyoshima ${ }^{3}$
}

${ }^{1}$ Department of Neurology, Akita University School of Medicine, Akita, Japan; ${ }^{2}$ Division of Neurology, Akita Red Cross Hospital, Akita, Japan; ${ }^{3}$ Medical Education Center, Akita University School of Medicine, Akita, Japan

\section{PURPOSE}

Spinocerebellar ataxias comprise a genetically and clinically heterogenous group of neurodegenerative disorders. Spinocerebellar ataxia type 6 (SCA6) and 16q linked autosomal dominant cerebellar ataxia (16q-ADCA) are the major types of inherited pure cerebellar ataxias in Japan. Initially, sensorineural hearing impairment is reported to be frequently associated in 16q-ADCA. On the other hands, horizontal gaze nystagmus is frequently associated in SCA6. But, clinical differences between these two types of pure cerebellar ataxia are minimum. For the efficient differentiation between these two types of cerebellar ataxias, we 
evaluated the decrease of the regional cerebellar blood flow (rCBF) using the statistical analysis of single photon emission computed tomography (SPECT). [Methods] Each five and three genetically proven SCA6 and 16q-ADCA patients were analyzed (7 patients were analyzed by easy Z-score imaging system (eZIS) using $99 \mathrm{mTc}-\mathrm{ECD}$ and one was analyzed by three-dimensional stereotactic surface projection (3D-SSP) using 123I-IMP). [Results] In this series, patients of 16q-ADCA were complicated with cerebral infarction, calcification of bilateral basal ganglia, or arachnoid cyst in posterior fossa. Decreases of rCBF clearly demonstrated by 3D-SSP and e-ZIS were different between patients with 16q-ADCA and SCA6. In 16q-ADCA patients, $\mathrm{rCBF}$ in lobulus semilunaris inferior was decreased predominantly. In contrast to $16 \mathrm{q}-\mathrm{ADCA}$, SCA6 patients showed decrement of rCBF in cerebellar vermis especially in lobulus centralis. [Conclusions] We should mention that 16q-ADCA patients frequently associated with other complications because ataxia of 16q-ADCA occurs in advanced age. SCA6 and 16q-ADCA could be discriminated by the rCBF decrease pattern showed in SPECT.

\section{PO5:2}

\section{RELATIONSHIP BETWEEN DIFFUSION TENSOR IMAGING AND BRAIN MORPHOLOGY IN PATIENTS WITH MYOTONIC DYSTROPHY}

N. Sato, M. Ota, Y. Ohya, Y. Aoki

Musashi Hospital, National Center of Neurology and Pshychiatry, Kodaira, Japan

\section{PURPOSE}

Myotonic dystrophy type 1 (MyD) is a common inherited neuromuscular disorder. In addition to neuromuscular symptoms, many MyD patients show central nerve system neuropathology. This study evaluated whether MyD patients display diffusion tensor (DT) abnormalities associated with regional cortical atrophy and clinical features.

MATERIALS AND METHODS

Three-dimensional T1-weighted (3DT1WI) and DT magnetic resonance images of the brain were obtained in $11 \mathrm{MyD}$ patients and 13 age- and sex-matched healthy subjects. Fractional anisotropy (FA) and mean diffusivity (MD) values were calculated in corpus callosum sub-regions with DT imaging (DTI) along with volumetric changes, and correlations with clinical features were examined. 3DT1WI images were analyzed using optimized voxel based morphometry (VBM) to assess the regional gray matter volume changes. Differences between MyD patient and healthy subjects were analyzed statistically.

RESULTS

Significantly lower FA and higher MD values were found in the genu, rostral body, anterior midbody, posterior midbody and splenium in MyD patients than in control subjects $(\mathrm{P}<0.05$, corrected. Lower FA in the splenium was at a trend level). These corpus callosum subregions were the areas connected to cortical areas where significantly lower volumes were found in MyD patients. No significant decrease in volumes was noted in the parietal cortex, where connecting fibers pass through the isthmus in which DTI abnormalities were not detected in MyD patients. Significant negative correlations to volume of frontal areas were noted, particularly bilateral motor areas, with cytosine thymine guanidine (CTG) triplet expansion.

\section{CONCLUSIONS}

DTI and VBM results in corpus callosum and cerebral cortex may reflect morphological changes in the connecting cortical areas of MyD patients.

\begin{tabular}{|c|c|c|c|c|c|}
\hline Genu & RB & AMB & PMB & Isthmus & Splenium \\
\hline \multicolumn{6}{|c|}{$\mathrm{FA}$ in MyD patients $\left(10^{-1} \%\right)$} \\
\hline $7.04 \pm 0.89^{\star}$ & $5.59 \pm 0.72^{\star}$ & $5.96 \pm 1.01^{\star}$ & $6.08 \pm 1.07^{\star}$ & $6.10 \pm 0.78$ & $7.73 \pm 0.71^{\star \star}$ \\
\hline \multicolumn{6}{|c|}{ FA in healthy subjects $\left(10^{-1} \%\right)$} \\
\hline $8.36 \pm 0.4$ & $16.98 \pm 1.16$ & $7.17 \pm 0.88$ & $7.35 \pm 0.75$ & $6.63 \pm 1.00$ & $8.37 \pm 0.43$ \\
\hline \multicolumn{6}{|c|}{ MD in MyD patients $\left(10^{-4} \mathrm{~mm} 2 / \mathrm{s}\right)$} \\
\hline \multicolumn{6}{|c|}{ MD in healthy subjects $\left(10^{-4} \mathrm{~mm} 2 / \mathrm{s}\right)$} \\
\hline $7.23 \pm 0.73$ & $8.62 \pm 2.06$ & $8.38 \pm 1.04$ & $8.46 \pm 0.88$ & $9.92 \pm 1.38$ & $3.46 \pm 0.78$ \\
\hline
\end{tabular}

RB. Rostal body: AMB. Anterior midbody: PMB. Posterior midbody * There was significant difference between MyD patients and healthy subjects. A p value There was significant difference between MyD patients
less than $0.008(=0.05 / 6)$ was considered significant.

* * There was difference between MyD patients and healthy subjects at a trend level $(P<$ $0.05)$

\section{PO5:3}

\section{MAGNETIC RESONANCE IMAGING IN ATYPICAL CREUTZFELDT-JAKOB DISEASE}

M. Ribeiro, A. Machado, J. Fernandes, M. Ma, C. Ferreira,

J. Rocha

Hospital Sao Marcos Braga Portugal

Magnetic Resonance imaging in atypical Creutzfeldt-Jakob disease

\section{INTRODUTION}

Creutzfeldt-Jakob disease (CJD) is a neurodegenerative disorder characterized by rapidly progressive dementia, myoclonia and ataxia. Magnetic Resonance imaging (MRI) plays an important role in early diagnosis.

CASE REPORT

A 47-year-old female presented a history of progressive cognitive deterioration over a period of one year, increasing during the last 3 months. Previous medical and familial histories were unremarkable. A cerebral MRI revealed gyriform high signal on FLAIR and DWI, slightly asymmetric (right predominance), involving the frontal, parietal, occipital and temporal lobes cortex. The basal ganglia had no abnormal signal intensity and no brain atrophy was observed. Electroencephalogram (EEG) did not show periodic activity. Cerebrospinal fluid (CSF) analysis, including 14-3-3 protein, was also negative. DNA workup revealed heterozigoty in codon 129, with no mutations in the PRP gene.

She continued to deteriorate becoming completely dependent with a MMSE of 10.

Cerebral MRI was repeated after 6 months and 1 year persisting gyri-like high signal, and in the latest one we also noted symmetric high signal at the bilateral putamen and head of caudate, and mild brain atrophy. 


\section{CONCLUSION}

The atypical clinical features with slower progression and absence of cerebellar and extrapyramidal signs (Alzheimer-like), associated with the absence of EEG periodic activity and 14-3-3 protein in the CSF, make this diagnosis especially challenging.

MRI has become progressively more relevant in dementia diagnosis; especially the diffusion-weighted imaging (DWI). Several studies have shown a $100 \%$ specificity in two radiological patterns of CJD (high signal in striatum and more than one gyri or wide high signal exclusively cortical). In this patient, the presumed diagnosis has only been possible by the imaging features, while WHO criteria were insufficient to establish it.

\section{PO5:4}

\section{MRI FINDINGS OF ADULT ONSET NIEMANN-PICK TYPE C}

\author{
M. Mendes ${ }^{1}$, D. Ferreira ${ }^{1}$, L. Lacerda ${ }^{2}$, G. Nadais ${ }^{1}$ \\ ${ }^{1}$ Hospital S.João, Oporto, Portugal; ${ }^{2}$ Instituto De Genética Médica \\ Jacinto Magalhães, Oporto, Portugal
}

\section{INTRODUCTION}

Niemann-Pick disease type $\mathrm{C}$ is an autosomal recessive neurovisceral lipid storage disease, resulting from mutations of the NPC 1 gene (95\% of families) or the NPC 2 . The disease shows a wide spectrum of clinical phenotypes and high variable age at diagnosis, ranging from the perinatal period to adult age.

The adult onset of Niemann-Pick is infrequent ( $5 \%$ of cases), slowly progressive, mostly characterized by neuropsychiatric signals.

The few brain MRI data available were normal or demonstrated white matter changes, cerebellar or cerebral atrophy, in relation with the clinical signals.

\section{CASE REPORT}

A 23 year-old-man presented with a 3 year history of clumsiness and poor coordination, associated with a slurred speech and cognitive deterioration. At neurological examination, there were cerebellar ataxia, dysarthria and a moderate degree of cognitive impairment. Brain MRI revealed severe cerebellar atrophy and slightly atrophy of the medulla oblongata. Thoracoabdominal CT demonstrated hepatosplenomegaly. Bone marrow examination was unremarkable.

The diagnosis of the classical form of NPC was confirmed by the low cholesterol esterification and storage of free cholesterol in lysosomes in cultured skin fibroblasts, detected by staining with filipin. The genetic molecular analysis showed NPC 1 mutation gene.

\section{DISCUSSION}

In spite of MRI findings being unspecific, we can conclude that there is a good correlation between the clinical features presented by our patient and the MRI study.

Niemann-Pick type $\mathrm{C}$ is a diagnosis that demands a high index of suspicious, with a time of delay for diagnosis of 6 years in some cases. Furthermore, the introduction of substrate reduction therapy, in order to reduce the lipid storage and prevent irreversible neurological lesions, makes brain MRI important for diagnosis and monitoring the clinical evolution of patients.

\section{P05:05}

\section{A VOXEL-BASED MORPHOMETRIC STUDY IN PATIENTS WITH MAJOR DEPRESSIVE DISORDER}

\section{K. Lee, S. Kim, J. Park, W. Tae, K. Kim}

Neuroscience Research Institute, Kangwon National University College, of Medicine Chuncheon, South Korea

\section{PURPOSE}

Voxel-based morphometry (VBM) is an automatic technique to allow the investigation of changes in volume and characteristic tissue concentration for the entire brain on a voxel by voxel basis. The purpose of this study was to detect structural abnormality of the brain in patients with major depressive disorder (MDD) with VBM. METHODS

3-Dimensional (3D) T1 volume images were obtained using coronal turbo field echo (TFE) technique in 20 female MDD patients according to DSM-IV criteria and in 20 female normal volunteers, respectively. Two groups were closely age matched (age range, 21-58 years; mean age, 39 years). With using SPM2 (Wellcome Department of Cognitive Neurology, London, UK), the VBM protocol was applied for the analysis of the brain tissue concentration and the regional volume change. After creating a study-specific T1 template and prior images, all the 3D volume images ( $\mathrm{n}=40$ scans) were normalized, segmented, and smoothed with an $8 \mathrm{~mm}$ full-width at half maximum.

\section{RESULTS}

The gray matter concentration was decreased bilaterally in hippocampus, fusiform gyrus, prefrontal areas, head of caudate nucleus, anterior and posterior cingulate gyrus in MDD patients (corrected $\mathrm{p}<0.05$ ). In addition, the volume change was found on the both side of hippocampus. No significant difference was observed in the white matter.

\section{CONCLUSIONS}

The finding of reduced volume in hippocampal regions in MDD patients is consistent with the high prevalence of memory disturbances, the domain of cognitive function that is frequently observed in patients with MDD. Of further note, abnormal gray matter concentration in the whole limbic system suggests that limbic circuits may be critical for mood regulation and may have a role in the pathophysiology of MDD.

\section{PO5:6}

MRI BASED MORPHOMETRY OF THE MEDIOTEMPORAL REGION STRUCTURES IN PATIENTS IN EARLY PHASE OF MAJOR DEPRESSIVE DISORDER

$\underline{\text { B. Goraj }}^{1}$, K. Van Oijen ${ }^{2}$, P. Van Eijndhoven ${ }^{2,3}$, M. Rijpkema ${ }^{3}$, M. Zwiers ${ }^{2,3}$, G. Van Wingen ${ }^{2,3}$, G. Fernandez ${ }^{3,4}$, I. Tendolkar ${ }^{2,3}$ ${ }^{1}$ Department of Radiology, University Medical Center Nijmegen, Nijmegen, The Netherlands; ${ }^{2}$ Department of Psychiatry, University Medical Center Nijmegen, Nijmegen, The Netherlands; ${ }^{3}$ F.C. Donders Centre for Cognitive Neuroimaging, University of Nijmegen, Nijmegen, The Netherlands; ${ }^{4}$ Department of Neurology, University Medical Center Nijmegen, Nijmegen, The Netherlands 
AIM

Decreased volume of the whole brain and of the hippocampi have been observed on MRI in patients with Major Depressive Disorder (MDD) whereas the changes in the volume of amygdala remain debatable. The aim of the present study was to evaluate the volume of both hippocampi and amygdala early during MDD in order to assess possible abnormalities which might occur already in the first stages of the disease.

METHODS

20 patients diagnosed with MDD and 20 controls, matched for age, gender and education underwent neuropsychological testing to account for distortions in attention, working memory and memory of verbal and non-verbal information. Patients were remitted from a first depressive episode for more than six months and not receiving any medication at the time of testing. 3-D T1weighted MP-RAGE sequence was acquired on a 1,5 Tesla scanner. Manual volumetry of amygdala and hippocampi was performed by two independant raters and combined with automatic segmentation (SPM5) of gray and white matter, cerebrospinal fluid and total brain volume.

RESULTS

No significant differences were found in the neuropsychological assessment and in the results of the automatic segmentation between the both groups The interrater reliability was 0,9 . Manual volumetry showed that across the groups right amygdala was signifcantly smaller than left, while the opposite was true for the hippocampus. Final pairwise comparisons indicated that left amygdala was smaller in the patient group only when not being corrected for multiple comparisons.

CONCLUSIONS

Though findings may be limited due to a too small sample size, evidence is emerging that structural changes of mediotemporal regions occur already early in the course of MDD independent of the state of the disease. Investigation of larger group of patients in the early phase of MDD and of the patient's familiy members could elucidate whether these abnormalities constitute the cause or consequence of MDD.

\section{PO5:7}

MRI CHARACTERISTICS IN ADULT-ONSET AUTOSOMAL DOMINANT LEUKOENCEPHALOPATY: A CASE REPORT

\author{
S. Battaglia ${ }^{1}$, A. Marliani ${ }^{1}$, M. Maffei ${ }^{1}$, F. Bartiromo ${ }^{2}$, \\ R. Agati ${ }^{1}$, M. Leonardi ${ }^{1}$ \\ ${ }^{1}$ Neuroradiology Department, Bologna, Italy; ${ }^{2}$ Federico Ii University, \\ Naples, Italy
}

\section{INTRODUCTION}

Dominantly inherited leukodistrophies with onset in adults are rare. They are progressive diseases of myelin genetically determined and have been described in multiple members of an American- Irish family. In this family the onset of clinical symptoms was in the fourth or fifth decade of life. Neurological manifestations were characterized by autonomic dysfunction, cerebellar- pyramidal dysfunction and mental deterioration. We describe the MR appearances of the brain in a member of an Italian family with adult-onset autosomal dominant leukoencephalopathy.

\section{CASE REPORT}

We report a case of a 52 years old woman affected by gait instability, uracrasia, visual fogging from clino to orthostatism.

Family history releaved: 58 years old mother deceased with diagnosis of "spino cerebellar heredo ataxia" (?), with three sisters and two brothers presenting the same symptoms. One brother of the patient is affected by adult-onset autosomal dominant leukoencephalopathy, the other one actually is asymptomatic. The patient have three sons: two (30 and $18 \mathrm{y}$ ) asymptomatic and one (24 y) deceased. The patient underwent morphological and functional cerebral MRI 3 Tesla that shows high T2 and low T1 signal intensity in cerebral white matter, particularly in frontoparietal regions, with relative preservation of the $U$ fibers, in the whole length of the pyramidal tracts from parietal lobe to medulla oblongata, in the upper and the middle cerebellar peduncles. All lesions are symmetrical. Cerebellar, cerebral and brainstem atrophy are also present.

Single-voxel Spectroscopy MR, performed in two areas of altered signal intensity (left middle cerebellar peduncle and right centrum semiovale), show presence of a small quantity of lipids, but normal relative concentrations of the principal metabolites.

CONCLUSIONS

The MRI findings are highly suggestive for adult-onset autosomal dominant leukodystrophy, due to affection of the corticospinal tracts and the presence of symmetrical extensive white matter changes, predominantly in the frontal and parietal lobes and cerebellum peduncles.

\section{PO5:8}

ASYMMETRICAL PERIVENTRICULAR HYPERINTENSITY ON AGED BRAIN WITH UNILATERAL MASS EFFECT

I. Anno $^{1}$, A. Kunimatsu, M. Hiratsuka, T. Isobe, M. Minami

${ }^{1}$ University of Tsukuba, Tsukuba, Japan; ${ }^{2}$ Kitazato University, Sagamihara, Japan

\section{PURPOSE}

Periventricular hyperintensity area on aged peoples is named as periventricular cup and usually considered to be non pathological finding. However, there are many pathologies in these lesions such as ischemia, gliosis, multiple sclerosis plaques, trans-ependymal CSF flow and so on. The purpose is to show an evidence of transependymal CSF flow, and discuss the characteristic diagnostic finding.

\section{METHOD AND MATERIALS}

Twenty cases of aged patients with unilateral chronic subdural hematoma were retrogradely analyzed. MRI were performed with axial T1, T2 weighted, FLAIR, and proton density weighted images. The size and shape of periventricular high intensity were classified. RESULTS

The size of the periventricular high intensity around the anterior horn of the lateral ventricles which is considered to be periventricular cup 
on the ipsilateral side of subdural hematoma was smaller than that of the contralateral side. High intensities those are obviously considered to be ischemic change was almost symmetrical. Post opertive MRI scan revealed that the periventricular cup returned to be symmetrical. This finding presumably represents that the high pressure gradient of the ipsilateral side of subdural hematoma blocks the trans-ependymal CSF flow or large subdural hematoma covering the surface of the brain obstruct the CSF absorption at the arachnoid granules.

\section{CONCLUSION}

We found one of the MRI evidence of trans-ependymal flow. Triangular shaped and cup like shaped high intensity areas around the anterior horn of lateral ventricles in aged people are indicating trans-ependymal CSF flow. These are presumably not pathological condition but normal aged brain.

\section{PO5:9}

\section{MR SPECTROSCOPY TO ASSESS NORMAL, MILD COGNITIVE IMPAIRMENT AND ALZHEIMER DISEASE}

R. Parodi ${ }^{1}$, L. Bonzano ${ }^{2}$, M. Pizzorno ${ }^{3}$, M. Abbruzzese $^{3}$, L. Castellan ${ }^{1}$

${ }^{1}$ Neuroradiology Department, San Martino University Hospital, Genoa, Italy; ${ }^{2}$ Magnetic Resonance Research Centre on Nervous System Diseases, University of Genoa, Genoa, Italy; ${ }^{3}$ Department of Neurosciences, Ophthalmology and Genetics, University of Genoa Genoa, Italy

\section{PURPOSE}

to evaluate different MR spectroscopy pattern of post-cingular gray matter among Normal Subjects (NS), Mild Cognitive Impairment (MCI) and Alzheimer Disease (AD) patients.

METHODS

16 Patients (age: mean $78 \mathrm{y}$, range 63-90 y) referred to our Dementia Evaluation Unit with suspected initial dementia (mild memory disorders) underwent the following clinical and MR spectroscopy (MRS) protocol. Clinical: a battery of Neurophycological Tests and in particular Mini Mental State Examination (MMSE), Clinical Dementia Rate (CDR), Hamilton Scale (testing mood disorders). Patients were categorized on the basis of the Clinical Demential Rate (CDR): $0=\mathrm{NS}$; $0.5=\mathrm{MCI} ; 1=\mathrm{AD}$. MRS: GE Signa Excite $(1.5 \mathrm{~T})$ equipment; spectroscopic acquisition of a $2 \times 2 \times 2 \mathrm{~cm}(8 \mathrm{~cm} 3)$ volume on the post-cingular bilateral gray matter with a PRESS sequence (TR $2000 \mathrm{~ms}$, TE $35 \mathrm{~ms}$, NEX 8). MRS data were post-processed using LCModel; we focused our interest on N-Acetyl-Aspartate (NAA) and Inositol (Ins) concentrations.

Statistical analysis was performed using Kruskal-Wallis Test and Linear Regression Model.

\section{RESULTS AND CONCLUSIONS}

Clinical results were: NS: $3 \mathrm{M}$ and $2 \mathrm{~F}$, age $75.6 \pm 5.6$ years (mean \pm SD); MCI: $2 \mathrm{M}$ and $5 \mathrm{~F}$ age $77.3 \pm 8.1$ years (mean $\pm \mathrm{SD}$ ); AD: $1 \mathrm{M}$ and $3 \mathrm{~F}$ age $81.0 \pm 5.4$ years (mean $\pm \mathrm{SD}$ ). MRS mean concentration ratio were: NAA/Ins 1.45 (CI 95\% 1.39-1.51) for NS, 1.21 (CI 95\%
1.11-1.31) for MCI and 1.29 (CI 95\% 1.06-1.52) for AD. LCModel software uses an acceptable reliability estimates with a standard deviation below $20 \%$ of the estimated concentrations. No lipid and lactate peaks were detected. Statistical difference $(p=0.03)$ was found for the NAA/Ins ratio among groups, while other data had no significant difference. Using a Regression Model, NAA/Ins could be a predictor $(\mathrm{p}=0.046)$ of categorization. In medical literature, the reduction of NAA and the increase of Ins were correlated to AD patient. Due to this fact, the NAA/Ins ratio could be a more stringent indicator. In our series NAA/Ins enabled the diversification between NS and MCI while the low number of AD (creating a large confidence interval) prevented any significant statistical evaluation. More patients have to be included to confirm this trend. To clarify the possible MRS pattern of conversion to AD, a follow up MRS study of MCI patients had been scheduled.

\section{Inflammatory and demyelinating disease}

\section{PO6:1}

\section{TRANSIENT FOCAL LESION IN THE SPLENIUM OF THE CORPUS CALLOSUM ON MR IMAGES: AN ATTEMPT TO CLINICAL AND PHYSIOPATHOLOGICAL EXPLANATION.}

\author{
M. Conti, A. Salis, C. Urigo, S. Frau, V. Manca, L. Canalis, \\ G. Canalis \\ Istituto Di Scienze Radiologiche, Sassari, Italy
}

\section{PURPOSE}

The aim of this report is to discuss the possible physiopathologic conditions responsible for these uncommon MR findings on the basis of our experience and a review of literature.

MATERIALS AND METHODS

In six patients, mean age 21.8 yo(16-34) who underwent MRI (Philips Intera Achieva $1.5 \mathrm{~T}$ ) examinations were incidentally detected focal nonhemorrhagic lesions of the splenium of the corpus callosum. In all patients the examinations have been repeated after 4,8 and 12 weeks and in two cases also after 6 and 9 months respectively. MR images and medical records were reviewed retrospectively with respect to the patients' clinical history, medication and laboratory findings to define the etiology of the lesions.

\section{RESULTS}

In all patients the detected lesions revealed to be focal, with no contrast enhancement and reversible. None of them had a history of antiepileptic treatment. In 4 patients the lesion disappeared after complete recovery of the underlying desease while in 2 patients the lesions persisted until to 6 and 9 months.

\section{CONCLUSIONS}

To our knowledge and according to other authors experiences, being these lesions detected in a relatively wide number of conditions with heterogeneous etiopathogenetic factors (infections, antiepileptic drugs, altitude, metabolic syndromes), it leads to suppose a common underlying physiopatologic mechanism, that 
considering signal characteristic, reversibility and white matter location, could be represented by AVP imbalance producing a vasogenic edema with a signal alteration within the splenium.

\section{PO6:2}

\section{PARRY-ROMBERG SYNDROME: REPORT OF TWO CASES}

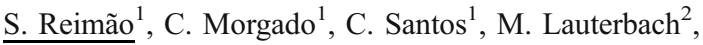
P. Santos ${ }^{3}$, J. Campos ${ }^{1}$

${ }^{1}$ Neurologic Imaging Department - Saint Mary's Hospital, Lisbon, Portugal; ${ }^{2}$ Neurology Department - Saint Mary's Hospital, Lisbon, Portugal; ${ }^{3}$ Radiology Department - Hospitalar Center of Coimbra, Coimbra, Portugal

\section{PURPOUSE}

Progressive Hemifacial Atrophy, also known as Parry-Romberg Syndrome, is an uncommon degenerative and poorly understood condition characterized by a slow and progressive atrophy affecting one side of the face. The incidence and the cause of the alteration are unknown. Ipsilateral hemiatrophy and calcifications are the most frequent brain abnormalities, with a wide range of lesions described, all presenting at the affected hemisphere. We report two cases, one of a 30 years old female and other of a 8 years old child, both presenting with marked hemifacial atrophy. Although the facial atrophy was more pronounced in the child, we found brain CT and MRI lesions in the homolateral hemisphere only in the young woman who presented with severe headache without neurological signs.

\section{CASE STUDIES}

Case 1: A 30 year-old female had a history of marked wasting of the right side of the face with onset at the age of 25 , progressing gradually over 4 years, static for the last year. There was no history of facial trauma and no family history of similar lesion. On examination, she had marked wasting of the right side of face with exaggerated temporal hollow, marked right enophthalmos and striking bony prominence. Case 2: An 8 year-old girl presented with exuberant wasting of the left side of the face, marked homolateral enophthalmos and striking bony prominence, progressing gradually over 3 years. Neurological examination made at admission was normal on both cases.

RESULTS

Brain CT and MRI were performed on both cases. The child's brain imaging studies were normal whereas in the 30 years old female we found lesions of the affected hemisphere including blurring of the cortical/sub-cortical interface and sulcal effacement at the occipito-parietal region, enlargement of the occipital horn and atrium of the lateral ventricle with $\mathrm{T} 2$ hiperintensity of the surrounding white matter and white matter calcifications predominantly in the corona radiata.

CONCLUSIONS

Parry-Romberg Syndrome's diagnosis is based on the clinical picture. Nevertheless we should be aware of the imaging findings that may be found particularly on brain scans. Various theories have been proposed to explain the pathophysiology of this rare disorder and its brain abnormalities but they remain uncertain. There is no definite treatment except plastic surgery to ameliorate cosmetic disfigurement.

\section{PO6:3}

DIFFUSION TENSOR (DTI), PERFUSION (PWI)

AND MAGNETIC RESONANCE SPECTROSCOPY

(MRS) TECHNIQUES IN MULTIPLE SCLEROSIS (MS) :

STUDY OF THE DIRTY WHITE MATTER(DWM)

E. Papadaki, N. Skoulikaris, S. Nikitidis, V. Mastorodimos, S. Karampekios, N. Papanikolaou, T. Maris, N. Gourtsoyiannis University Hospital of Heraklion, Heraklion, Greece

\section{PURPOSE}

Application of the DTI, PWI and MRS techniques in patients with MS, for the better characterization of the DWM regions.

\section{MATERIAL AND METHODS}

16 patients with relapsing-remitting MS were studied on a $1.5 \mathrm{~T}$ MR scanner. A T2SE-EPI sequence with 6 different diffusion gradients, a single shot T2* GRE-EPI sequence with 50 dynamic axial scans after a single dose of Gd administration, and a 2D PRESS multivoxel sequence were applied for the DTI , PWI and MRS, respectively. 244 measurements of the apparent diffusion coefficient (ADC), fractional anisotropy (FA), relative cerebral blood volume (rCBV), N-acetylaspartate/Creatine (NAA/Cr), Myoinositol/Creatine $(\mathrm{mI} / \mathrm{Cr})$ and Choline/Creatine $(\mathrm{Cho} / \mathrm{Cr})$ values were performed, concerning normal appearing white matter (NAWM=76), DWM(46) and focal white matter lesions (122). Possible statistically significant differences among the ADC, FA, $\mathrm{rCBV}, \mathrm{NAA} / \mathrm{Cr}, \mathrm{mI} / \mathrm{Cr}$ and $\mathrm{Cho} / \mathrm{Cr}$ values of the measured white matter regions were evaluated, by using student's t-test.

\section{RESULTS}

DWM regions demonstrared significantly higher $\mathrm{rCBV}(\mathrm{p}=0.02)$, ADC $(p<0.0005)$, and $\mathrm{mI} / \mathrm{Cr}(\mathrm{p}<0.0005)$ values and lower FA $(p=0.02)$ values compared to NAWM and significantly different values compared to the most of the focal white matter lesions. There was no significant difference between the DWM's CBV, $\mathrm{ADC}, \mathrm{FA}, \mathrm{NAA} / \mathrm{Cr}$ and $\mathrm{Cho} / \mathrm{Cr}$ values and the respective values of the focal isointense, on T1 sequence, lesions(=ISO), while mI/ $\mathrm{Cr}$ values of the DWM were significantly higher $(\mathrm{p}=0.04)$ compared to that of the ISO.

\section{CONCLUSION}

DWM regions demonstare high regional blood volume, increased diffusivity, decreased fractional anisotropy, and increased $\mathrm{mI} / \mathrm{Cr}$ values, while they don't reveal significant differences compared to the active focal while matter lesions, with the exception of the $\mathrm{mI} /$ $\mathrm{Cr}$ ratio..These findings probably reflect diffuse inflammation and demyelination at the DWM regions that proceed the acute active focal lesions formation. 


\section{PO6:4}

\section{MR IMAGING OF LIMBIC ENCEPHALITIS. REPORT OF A CASE}

S. Lyra ${ }^{1}$, A. Kokkinaki ${ }^{1}$, A. Papatheodorou ${ }^{1}$, P. Ellinas ${ }^{1}$, A. Panousopoulou ${ }^{2}$, N. Batakis ${ }^{1}$

${ }^{1}$ Radiology Department Red Cross Hospital, Athens Greece; ${ }^{2}$ Neurology Department Red Cross Hospital, Athens Greece

\section{PURPOSE}

Limbic encephalitis is a rare entity affecting the limbic system. Most of the times it is a paraneoplasmatic syndrome observed in patients with small cell carcinoma and gastrointestinal and genitourinary tumors. Nevertheless in many cases no underlying malignancy is detected at workup.

We present a case of limbic encephalitis and discuss the clinical signs and its MR Imaging findings.

METHODS

A 67 year old female, heavy smoker, was admitted to our radiology department because of mental deterioration during the last few weeks. Her medical history was unremarkable. We performed CT examination of the brain before and after contrast administration, as well as MR examination. The MR protocol included T2 turbo spin echo weighted images (T2TSEWI), flow attenuated invasion recovery (Flair), T2* gradient echo (GRE), diffusion DWI as well as T1WI and T1WI after intra venous (IV) administration of Gadolinium (Gd).

RESULTS

The CT evaluation revealed no specific findings except for chronic hypertensive leukoencephalopathy. The MR examination revealed hyperintensity in mesial temporal lobes at T2WI and Flair images and minimal hypointensity at T1WI. No hemorrhage was seen. After IV administration of $\mathrm{Gd}$, patchy enhancement was observed at the lesion of the left side. The patient was referred for further evaluation which did not reveal any primary neoplasm. Patient's health status improved after IV administration of immunoglobulin and corticosteroids.

CONCLUSIONS

MR examination is the imaging modality of choice for the diagnosis of limbic encephalitis.

\section{PO6:5}

\section{IMPROVED LESION DETECTION WITH LATE CONTRAST-ENHANCED IMAGES WITH GADOVISTR}

D. Nguyen, F. Zeccolini, T. Wels, H. Jlassi, M. Vargas,

J. Delavelle, K. Lovblad

HUG, Neuroradiology, Geneva, Switzerland

\section{INTRODUCTION}

Gadobutrol (Gadovist ${ }^{\circledR}$ 1.0) is a high-relaxivity, 1.0 molar MR contrast agent. We wanted to compare its ability to improve MS lesion detection at late imaging time points post-injection to that of
Gadodiamide $\left(\right.$ Omniscan ${ }^{\circledR}$ ), which is provided at an $0.5 \mathrm{M}$ concentration.

\section{MATERIAL AND METHODS}

Eighteen patients with suspicion of or known MS were included. MRI was performed with axial $\mathrm{T} 1$ and $\mathrm{T} 2 \mathrm{w}$ images as well as sagittal FLAIR images. Patients were randomly assigned to receive either Gadobutrol (11 patients) or Gadodiamide (7 patients) . Post-contrast MRI of the brain was performed 3, 6, and 9 minutes after i.v. injection of the contrast agent to obtain axial T1w (SE) images. Additionally, one patient underwent MRI of the spine (sagittal T1w MRI). Quantitative measurements of signal intensities (ROIs included lesions, brain tissue outside of the lesions, controlaterally with a total of 8 measurements per time-point) was performed on an outboard console. In addition qualitative assessment of signal intensities was also performed RESULTS

Twelve patients had a definitive diagnosis of multiple sclerosis, five out of which were found to have contrast-enhancing lesions. Seven patients had non-enhancing typical MS lesions; of the five patients, four received Gadobutrol and one received Gadodiamide. An average number of 5 lesions were seen per patient. With Gadobutrol, a continuous increase in lesion enhancement was observed on the images obtained at 3,6 , and 9 minutes postinjection. For Gadodiamide no further increase of lesion enhancement was observed beyond 6 minutes postinjection. Qualitative assessment of contrast enhancement also resulted in higher values for Gadobutrol in comparison to Gadodiamide.

\section{CONCLUSIONS}

In comparison to Gadodiamide a higher increase of enhancement of active lesions was seen. A continous contrast enhancement increase at later imaging time points was observed with Gadobutrol both for the quantitative as well as qualitative evaluation, but not with Gadodiamide.

\section{PO6:6}

\section{COMPARISON OF MR FINDINGS AND CLINICAL FEATURES OF OSMOTIC DEMYELINATION SYNDROME ACCORDING TO UNDERLYING DISEASE IN METABOLIC ENCEPHALOPATHY.}

H. Lee ${ }^{1}$, H. Kim ${ }^{2}$, S. Baek ${ }^{1}$, Y. Kim ${ }^{1}$

${ }^{1}$ Department of Radiology, Kyungpook National University Hospital, Daegu, South Korea; ${ }^{2}$ Department of Radiology, Daegu Catholic University Medical Center 1, Daegu, South Korea

\section{BACKGROUND AND PURPOSE}

Although osmotic demyelination syndrome was proved as concurrent with rapid correction of hyponatremia and osmotic shift by means of clinical and experimental work, it is very difficult to diagnose and treat because of its various underlying causes and clinical manifestations. Our purpose was to clarify the cause of osmotic demyelination syndrome by evaluation of MR findings and clinical features. 


\section{METHODS}

We reviewed MR images and clinical features for 25 patients who showed abnormal signal changes on pontine or extrapontine area with neurologic deterioration.

RESULTS

The 25 patients had underlying diseases such as chronic liver disease $(n=16)$ or renal failure $(n=9)$. The MR findings showed significant differences in the involved sites according to the underlying disease. In 10 of the 16 patients with liver disease, corpus callosal involvement was observed. Red nucleus involvement was seen in 6 patients, dentate nucleus involvement in 5 patients. Contrary to liver disease, encephalopathy with renal disease showed typical central pontine myelinolysis in 6 of the 9 patients. The patients with hepatic failure had more poor clinical outcome than with renal problem.

\section{CONCLUSION}

Our results showed that the typically involved site and clinical manifestations depended on the underlying disease. We think that involvement of the corpus callosum, the red nucleus, and the dentate nucleus is a typical pattern of injury with chronic liver disease and that these findings will be helpful for diagnosing and treating metabolic encephalopathy.

\section{PO6:7}

\section{NON-PARANEOPLASTIC LIMBIC ENCEPHALITIS ASSOCIATED WITH SYSTEMIC LUPUS ERYTHEMATOSUS}

A. Kunimatsu $^{1}$, M. Hiratsuka ${ }^{2}$, I. Anno ${ }^{1}$, N. Kunimatsu ${ }^{3}$, M. Minami $^{1}$

${ }^{1}$ Institute of Clinical Medicine, University of Tsukuba, Tsukuba, Japan; ${ }^{2}$ Tsukuba University Hospital, Tsukuba, Japan; ${ }^{3}$ Graduate School of Medicine, University of Tokyo, Tokyo, Japan

\section{BACKGROUND}

Up to $95 \%$ of patients with systemic lupus erythematosus (SLE) develop neuropsychiatric manifestations during the course of the disease which are designated as neuropsychiatric SLE (NPSLE). Limbic encephalitis is clinically characterized by cognitive dysfunction, seizures, and psychiatric features, and is radiologically characterized by $\mathrm{T} 2$ signal hyperintensity at the mesial temporal lobes. Although it most often occurs in patients with malignancy, limbic encephalitis associated with non-neoplastic disease has been reported. Non-paraneoplasitc limbic encephalitis is extremely rare in NPSLE with only one case report available to date. We present NPSLE cases that showed T2 signal abnormalities at the mesial temporal lobes mimicking typical findings of limbic encephalitis on MRI to discuss features of MRI and clinical manifestations.

\section{METHODS}

Out of 72 SLE patients who underwent MRI with suspicion of NPSLE between May 2004 and October 2006 at our institution, four female patients showed T2 signal hyperintensity at the mesial temporal lobes and their imaging characteristics were reviewed. Clinical information was collected from medical records.

\section{RESULTS}

The clinical courses or laboratory tests excluded Herpes encephalitis and paraneoplastic limbic encephalitis. None of the four patients had a history of epilepsy or Hashimoto's thyroiditis. Two out of the four patients complained of headache, another patient left hemianopia, and the other visual disturbance. T2 signal hyperintensity was identified at the hippocampus in all patients; the amygdala in two; the parahippocampal gyrus in two; the insula and the subinsular white matter in two. Three patients showed bilateral and symmetrical involvement, but the other right dominant. Contrast enhancement was noticed in two.

\section{CONCLUSION}

It seems important for radiologists to know that NPSLE not uncommonly mimics limbic encephalitis on MRI and that such MR abnormalities may be present even with no history of epilepsy.

\section{PO6:8}

\section{CLINICOPATHOLOGICAL CORRELATIONS OF TUMEFACTIVE BRAIN LESIONS}

M. Kobayashi ${ }^{1}$, Y. Ono ${ }^{2}$, T. Maruyama ${ }^{3}$, Y. Muragaki ${ }^{3}$, O. $\mathrm{Kubo}^{3}$, T. Hori ${ }^{3}$, K. Abe ${ }^{2}$, M. Iguchi ${ }^{2}$, A. Hiroi ${ }^{4}$, N. Shibata ${ }^{4}$, M. Kobayashi ${ }^{4}$, K. Maruyama ${ }^{1}$, K. Miyazaki ${ }^{1}$, M.

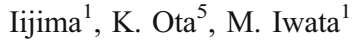

${ }^{1}$ Department of Neurology, Tokyo Women's Medical University, School of Medicine, Tokyo, Japan; ${ }^{2}$ Department of Neuroradiology, Tokyo Women's Medical University, School of Medicine, Tokyo, Japan; ${ }^{3}$ Department of Neurosurgery, Tokyo Women's Medical University, School of Medicine, Tokyo, Japan; ${ }^{4}$ Department of Pathology, Tokyo Women's Medical University, School of Medicine, Tokyo, Japan; ${ }^{5}$ Tokyo University of Science, Tokyo, Japan

\section{PURPOSE}

Comparison of histopathological and radiiolgical findings of tumefactive multiple sclerosis(TMS) to prove the pathogenesis of the radiological findings

\section{CASES AND METHODS}

Radiological and microscopic findings of 3 cases $(25,35,44$ yearold, male 2, female 1) with focal neurological signs and increased intracranial pressure were discussed retrospectively.

MRI findings(using 1.5 T, T1WI, T2WI, Gd-T1WI, DWI and MRS) showed tumor like findings, which are needed for differential diagnosis between TMS, glioma, lymphoma or abscess.

Because of rush progression of clinical symptoms, stereotactic needle biopsy or surgical resection was planned using computer navigated operation system.

The specimens were investigated carefully by histochemistry with hematoxylin-eosin, Klüver Barre, silver stain and immunohistoche- 
misty for vascular endothelial growth factor(VEGF) and macrophage chemotactic protein-1(MCP-1).

MRI and MRS findings were compared to these histological findings.

RESULTS

On MRI, 1) solitary large lesion, 2) lesion with high intensity in the center, inhomogeneous low intensity rim and surrounding edema with relatively less mass effect, 3) one case showed hemorrhage inside, 4) atypical open ring or deformed ring enhancement, 5) high intensity on DWI, 6) prominently decreased NAA/Cho ratio, without increase of lipid or lactate on MRS.

Macroscopic: no swelling of the brain surface. Microscopic: demyelination and angioneogenesis in 2 cases, prominent inflammatory cell aggregation along the capillary veins with thromobus inside in 1 case of hemorrhage, marked angioneogenesis at the periphery of the involved area with inflammation in 1 case, proved by positive VEGF and MCP-1.

The final diagnosis with these radiological and histopathological findings was TMS.

CONCLUSIONS

TMS is difficult to distinguish from tumors on image diagnosis, even with DWI and MRS.

The histopathological findings may explain edema formation and marked inflammatory cell infiltration. Inhomogeneously low intensity on T2WI at the periphery of the lesion is generally considered as the sign of acute inflammatory demyelination, additionally this report presents that angioneogenisis plays another role of formation of the MRI findings in TMS.

\section{PO6:9}

\section{ACUTE NECROTIC HEMORRHAGIC ENCEPHALITIS OF THE THALAMI AND DIFFERENTIAL DIAGNOSIS}

E. Kapsalaki, G. Protogerou, E. Dardiotis, A. Markopoulou, I. Fezoulidis

University Hospital of Larissa, Larissa, Greece

\section{PURPOSE}

The differential diagnosis of bilateral thalamic lesions is quite extensive. In children, acute disseminated encephalomyelitis (ADEM) involving the subcortical white matter and rarely the thalami occurs secondary to upper respiratory or gastrointestinal infection. CASE REPORT

We present a case of a 16 year old female referred to our hospital with left pyramidal signs and lethargy, progressing to coma on admission. A history of a gastrointestinal infection with fever, severe emesis and diarrhea 7 days earlier was reported.

The initial CT scan of the brain revealed areas of low densities in the thalami bilaterally. CT scan 24 hours later showed extension of the lesions to the basal ganglia as well as mild diffuse cerebral edema. An MRI of the brain confirmed the presence of the lesions at the thalami and extension to the periventricular white matter as well as the right cerebral peduncle. The initial diagnosis was deep venus thrombosis due to dehydration. However, an MRV excluded that diagnosis. The diagnosis of ADEM was proposed. The CSF laboratory results were compatible with ADEM (mild pleocytosis and elevated protein, negative PCR studies as well as negative bacterial and fungus cultures). She was placed on high-dose steroids. Her symptomatology slightly improved and follow-up MRIs of the brain showed resolution of the white matter edema but persistence of the thalamic lesions, with the presence of necrotic areas. These findings have been described as an evolution of ADEM to necrotic hemorrhagic encephalitis (ANHEM).

CONCLUSION

The radiological findings of this case, the associated differential diagnosis as well as a brief review of the pertinent literature will be fully presented.

\section{PO6:10}

\section{RARE REVERSIBLE MRI FINDINGS OF THE BRAIN IN A CASE OF ANTINEUTOROPHIL CYTOPLASMIC ANTIBODIES (ANCA) ASSOCIATED VASCULITIS}

M. Iguchi $^{1}$, Y. Ono ${ }^{1}$, M. Kobayashi ${ }^{2}$, H. Yoshizawa ${ }^{2}$, K. Abe ${ }^{1}$, S. Uchiyama ${ }^{2}$, M. Iwata

${ }^{1}$ Department of Neuroradiology, Tokyo Women's Medical University, School of Medicine, Tokyo, Japan; ${ }^{2}$ Department of Neurology, Tokyo Women's Medical University, School of Medicine, Tokyo, Japan

\section{PURPOSE}

To investigate correlation between rare reversible MRI findings and clinical course in a case of ANCA associated vasculitis. CASE AND METHODS

A 75-year-old man repeated high fever with unknown cause and diagnosed as myeloperoxidase (MPO)-ANCA associated vasculitis with elevation of MPO-ANCA, saddle nose and interstitial pneumonia. He has been followed by steroid therapy, but gradually developed dementia for several months. He was admitted with drowsiness and gait disturbance. He revealed progressed consciousness disturbance, continuous fever, hyperreflexia and pathological reflex in both lower extremities. With slight elevation of protein in cerebrospinal fluid, high level of CRP and uneventful other laboratory data, aggravation of ANCA-assocated vasculitis was suspected. By increased dosage of prednisolone, fever, CRP and drowsiness were alleviated. Serial MRI (T1WI, T2WI, Gd-T1WI, DWI, MRA) were compared to the clinical symptom/signs and meanings of the images were discussed.

RESULTS

MR images are as follows. On the 1st MR with clear consciousness; hydrocephalus, a HI spot in right fronto-orbital area (rFOA), small $\mathrm{HI}$ in pons on T2WI and slight stenosis of right middle cerebral artery(MCA). On the 2nd day of drowsiness; enlarged HI in $\mathrm{rFOA}$, appearance of $\mathrm{HI}$ areas in right lower frontal subcortex (rLFSC) on T2WI, remarkable stenosis of bilateral MCA and anterior cerebral arteries (ACA) on MRA, HI in rLFSC, right corona radiate and pons, but low intensity in rFOA on DWI. 1 month later with progressed drowsiness; clearer $\mathrm{HI}$ areas in rFOA, rLFSC and pons on T2WI. 4 month later with increased steroid and recovery of consciousness; $\mathrm{HI}$ in rFOA and rLFSC 
were disappeared and smaller $\mathrm{HI}$ are remained in pons and right corona radiate.

\section{CONCLUSION}

Reversible HI on T2WI and DWI with stenosis of the arteries of this case was considered to be mainly effected by continuous inflammatory process rather than ischemic changes.

\section{PO6:11}

\section{THE CEREBERELLAR FORM OF PROGRESSIVE MUTLIFOCAL LEUKOENCEPHALOPATHY WITHOUT IMMUNODEFICIENCY}

T. Hayashi, A. Wada, Y. Tsuchie, T. Katsube, H. Kitagaki Shimane university Faculty of Medicine, Izumo, Japan

PML (progressive mutlifocal leukoencephalopathy) most frequently appears in the hemispheric subcortical white matter.

The MR imaging of the isolated posterior fossa involvement has been less described in PML, especially there was no report following the course of PML on serial diffusion weighted images (DWI).

We present a new rare case of isolated posterior fossa involvement of PML without immunodeficiency, following the course of PML on DWI, and the review of the literature.

CASE

A 73-years-old man with a history of polycythemia vera was admitted to our hospital with dysarthria and right ataxia. After the admition, dysphagia and gait disturbance were gradually developing and progressing. The hematologic examination showed no evidence of immmunosupression state. Eventually, we had diagnosed PML because JC virus was detected in the cerebrospinal fluid by Polymerase Chain Reaction based demonstration of JC virus DNA.

The initial MR images demonstrated focal white matter signal abnormalities without mass effect and perifocal edema at right cerebellum.

DWI demonstrated hyperintense area on the forward part of the lesion with low ADC values. Serial MRI revealed the hyperintense area extending to the left cerebellum through the brain stem.

Serial imaging studies, especially DWI showed the aggressive nature of PML.

\section{PO6:12}

IMAGING INHERITED LEUKODYSTROPHY: NEW DATA FROM A FAMILY WITH ORTHOCHROMATIC LEUKODYSTROPHY

M. Hauf $^{1}$, R. Buehler ${ }^{2}$, C. Stuker ${ }^{1}$, J. Slotboom ${ }^{1}$, C. Kiefer $^{1}$, R. Wiest ${ }^{1}$

${ }^{1}$ Institute of Diagnostic and Interventional Neuroradiology, Berne, Switzerland; ${ }^{2}$ Departement of Neurology, Berne, Switzerland

\section{PURPOSE}

Leukodystrophies are inherited white matter diseases due to abnormalities occurring in myelin synthesis and/or maintenance. MR imaging has become the primary imaging modality in patients with leukodystrophy, although the results are often non-specific. New MR techniques as Diffusion Tensor Imaging (DTI) and MRSpectroscopy (MRS) may permit a narrower differential diagnosis. This is the first report of DTI and MRS images in a family with a pigmentary form of orthochromatic leukodystrophy.

\section{METHODS}

Two patients of a kindred with a history of 4 affected subjects underwent structural MR, DTI and MRS at a Siemens Sonata $1.5 \mathrm{~T}$ whole body scanner. The MR protocol included a T1MPRAGE-Sequence $(\mathrm{TR}=2000 \mathrm{~ms}, \mathrm{TE}=3.93 \mathrm{~ms}$, matrix $256 \times$ 256, FA 15), a spinecho EPI-sequence for DTI acquisition (TR= $3700 \mathrm{~ms}, \mathrm{TE}=87 \mathrm{~ms}$, matrix $96 \times 128$ in 6 diffusion encoding gradient directions) and H1-MR-Spectroscopy (single voxel PRESS, TE $=135 \mathrm{~ms}$ and $30 \mathrm{~ms}$ and a 2-D CSI-Sequence).

\section{RESULTS}

Clinically subjects present with epileptic seizures, frontal disinhibition behavior and diffuse cognitive decline starting at the age of 40, fatal within 3 years in one case. Structural MRI revealed symmetric bifrontal subcortical T1w hypo/ T2w hyperintensities with relative sparing of the U-fibers. Rupture of the blood/brainbarrier was not a feature. Quantitative and voxel-based DTI showed a markedly reduced anisotropy in the frontal lobes as the hallmark of focalised subcortical fiber degeneration. Neuronal degeneration was confirmed by NAA reduction in MRS in the frontal lobes. Elevation of Choline is in keeping with ongoing demyelisation.

CONCLUSION

For the first time this series characterises white matter alterations by DTI and MRS in a pigmentary form of orthochromatic leukodystrophy. The pattern of decreased anisotrophy restricted to the frontal lobes with relative sparing of the U-fibers has not yet been reported in other forms of leukodystrophies. As a responsible gene is not identified, MR changes have to be considered in genetic counselling.

\section{PO6:13}

POSTERIOR REVERSIBLE ENCEPHALOPATHY SYNDROME (PRES) IN INTENSIVE CARE MEDICINE PATIENTS

U. Dietrich $^{1}$, R. Borgstedt ${ }^{2}$, C. Hagemeister ${ }^{3}$, M. Zahran ${ }^{1}$, ${ }_{\text {F. Hermann }}{ }^{4}$, F. Bach ${ }^{2}$

${ }^{1}$ Neuroradiology, Bielefeld, Germany; ${ }^{2}$ Anaethesiiology, Bielefeld, Germany; ${ }^{3}$ Neurology, Bielefeld, Germany;

${ }^{4}$ Internal Medicine, Bielefeld, Germany

\section{PURPOSE}

Ten patients developing PRES during intensive care medicine treatment were observed within the last three years. The role of magnetic resonance imaging (MRI) to establish correct diagnosis was evaluated retrospectively. 


\section{METHODS}

Ten patients with PRES (eight females, two males, age: 27 to 73 years, mean 47.2) were treated with following underlying diagnoses: intracranial hemorrhage $(n=3)$, eclampsia $(n=2)$, pneumonia $(n=2)$, surgery of a tumor $(n=2)$ and hypertensive crisis $(n=$ 1). PRES clinically manifested with seizures in six cases and visual disturbances and/or alteration of vigilance in four cases. Eight patients had computed tomography (CT) and MRI in combination, one of them conventional angiography in addition. Two patients had either only CT or only MRI.

RESULTS

In two patients who had either only CT or only MRI, diagnosis of PRES was confirmed clinically. CT and MRI were equivalent in two of eight patients who had both procedures. In four of eight patients MRI was superior to CT demonstrating symmetry of lesions on FLAIR and of vasogenic edema on DWI sequences. CT failed to show correct diagnosis in these cases or was misinterpreted.

Diagnosis of the remaining two patients could only established using additional diagnostic tools: In one patient with pneumonia and sepsis, differential diagnosis of acute demyelinating encephalomyelitis (ADEM) could only be ruled out by repeated lumbar puncture. And in one patient with eclampsia, increased flow velocity of intracranial vessels was detected sonographically and digital subtraction angiography revealed vasospasm. Therefore diagnosis of Call-Fleming-Syndrome leading to PRES had been made.

\section{CONCLUSIONS}

MRI should be the first diagnostic tool in patients with clinical suspicion of PRES. CT may deliver findings which could be misinterpreted. There are only a few patients left who require additional clinical, laboratory or neuroradiological investigations.

\section{PO6:14}

\section{CERVICAL PROTON MAGNETIC RESONANCE SPECTROSCOPY IN EVOLUTIVE STUDY} OF RELAPSING-REMITTING MULTIPLE SCLEROSIS: A CASE REPORT.

L. Albini Riccioli ${ }^{1}$, A. Marliani ${ }^{1}$, V. Clementi ${ }^{2}$, I. Bartolomei ${ }^{3}$, R. Agati $^{1}$, M. Leonardi ${ }^{1}$

${ }^{1}$ Neuroradiology Department, Bellaria Hospital, Bologna, Italy; ${ }^{2}$ GE Healthcare, Bologna, Italy; ${ }^{3}$ Neurology

Department, Bellaria Hospital, Bologna, Italy

\section{PURPOSE}

the use of proton spectroscopy (1H-MRS) to evaluate mean relative concentrations ratios for NAA, $\mathrm{Cr}$, Cho and $\mathrm{mI}$ during the study of cervical spinal cord plaques in a relapsing-remitting multiple sclerosis (RRMS).

METHODS

A cervical spine MR exam was repeated 5 times on the following days for two months starting from the onset of a relapse phase., using a $3 \mathrm{~T}$ whole-body system.
1H-MRS VOI was prescribed along the main axis of the cord between the $\mathrm{C} 2-\mathrm{C} 3$ levels, on the cervical plaque hyperintense on T2 weighted images.

A PRESS and CHESS sequences were used with six saturation bands contiguously to the VOI to minimize fat contamination.

MRS data were analysed by the user-independent fitting routine LCModel and the main metabolites ratios were compared with the healthy metabolites contents.

RESULTS

The plaque revealed a slight different contrast enhancement on the 5 MR sessions. The quantification results, respect to the healthy metabolites ratios, show an initial phase with an increase of Cho suggestive of a demyelination and inflammatory process. The Cho will return on the normal range only on the last examination, 58 days after the onset. At 16 days from the offset $\mathrm{mI}$ increases. These changes are followed by a transient decrease of the tNAA, suggesting of secondary axonal/neuronal disfunction. 58 days after the onset all the metabolic ratios follow inside the healthy range. DISCUSSION

In our knowledge this is the first study in a cervical SM plaque of the temporal metabolic changes. The evolution of the observed plaque can be compared with similar trends on MS brain plaques. The quantitative cervical spectroscopy is a reliable tool and it can offer an important contribution of the metabolic information as already used on the brain to evaluate the severity, progression and pathogenesis of the MS.

\section{PO6:15}

\section{THE CONTRIBUTION OF MR IMAGING IN THE STUDY OF SPONDYLOARTHRITIS}

\author{
P. Pignataro $^{1}$, A. Tarantino ${ }^{2}$, P. D'Aprile ${ }^{2}$ \\ ${ }^{1}$ U.O. di Radiologia Ospedale "San Giacomo", Monopoli (BA),
} Italy; ${ }^{2}$ U.O.S. di Neuroradiologia Ospedale "San Paolo", Bari Italy

\section{PURPOSE}

to determine with magnetic resonance imaging (MRI), by using T2-weighted turbo spin-echo fat saturated and T1-weighted spinecho fat saturated sequences after injection of a contrast agent (gadolinium), spinal inflammation (also in early stage) in patients with suspect of rheumatic spondyloarthritis (SpA).

\section{METHODS}

we have studied in the period from 2005 june to 2007 may, with MR of $1.5 \mathrm{~T}$ Magnetom, the spine of 245 patients with clinical picture of spondyloarthritis or unknown active inflammatory back pain. The mean age was 39.7 years, $55 \%$ were male. MRI examination included axial and sagittal T1-weighted spin-echo and T2-weighted turbo spin-echo fat saturated sequences. In selected case, patients were subjected to T1-weighted spin-echo fat saturated sequence after injection of a contrast agent (gadolinium).

RESULTS

209/245 (85,3\%) patients showed different inflammatory lesions of the spine. Spinal changes associated with spondyloarthritis are: bone marrow edema (osteitis), florid anterior and posterior 
spondylitis, spondyldiskitis, arthritis (apophyseal, costovertebral and costotransverse joints) and enthesitis of the supraspinal and interspinal ligaments. The contrast agent showed clearly lesions, particularly in soft tissue, obtaning important informations about activity of disease.

\section{CONCLUSIONS}

with specialized MRI techniques, such as FAT SAT TSE T2 and FAT SAT SE T1 after injection of a contrast agent (gadolinium), we are able to detect spinal inflammation, also in early stage, with a high level of precision, so patients take advantage of the following pharmacological treatment.

\section{Tumors}

\section{PO7:1}

\section{ATYPICAL MENINGIOMA OF THE TRIGEMINAL NERVE IN A PEDIATRIC PATIENT}

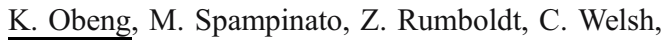

N. Besenski

${ }^{1}$ Medical University South Carolina, Charleston, USA

\section{PURPOSE}

Primary tumors of the trigeminal nerve and Meckel's cave are uncommon and represent less than $0.5 \%$ of intracranial tumors. It is an even less common occurrence in children. We report a case of an atypical meningioma of the left trigeminal nerve without dural attachmentn in an adolescent patient.

\section{METHODS}

We reviewed the clinical records of a 14 year-old Caucasian female who was admitted to our institution with a 2-month history of left-sided facial numbness and paresthesias of the left side of her tongue. The patient underwent an MRI of the brain. Based on the imaging and clinical findings, the patient underwent surgery. The MRI of the brain and pathology records were also reviewed. RESULTS

The brain MRI revealed a rim enhancing extra-axial dumbbellshaped cystic mass involving Meckel's cave and extending into the posterior fossa, along the course of the left trigeminal nerve. Given the imaging appearance, the mass was felt to be consistent with a cystic schwannoma of the left trigeminal nerve. Surgery was performed using an extra-dural skull base approach to resect this trigeminal mass. At surgery it was noted that the mass was arising from the trigeminal nerve, was intimately associated with the V1 and V2 branches of the trigeminal nerve, and did not have a dural attachment. The histology showed concentric structures typical of meningioma, but hypercellularity, focal necrosis and mitotically active foci. The final diagnosis was atypical meningioma. The tumor likely originated from arachnoid cap cells within the left trigeminal nerve sheath or from the perineural cells of the nerve and not from the dura of Meckel's cave.

\section{CONCLUSION}

Atypical meningiomas (World Health Organization grade II/IV) exhibit a more aggressive behavior and an increased risk of recurrence after treatment. To our knowledge, this is the first documented atypical meningioma originating from the trigeminal nerve in a pediatric patient.

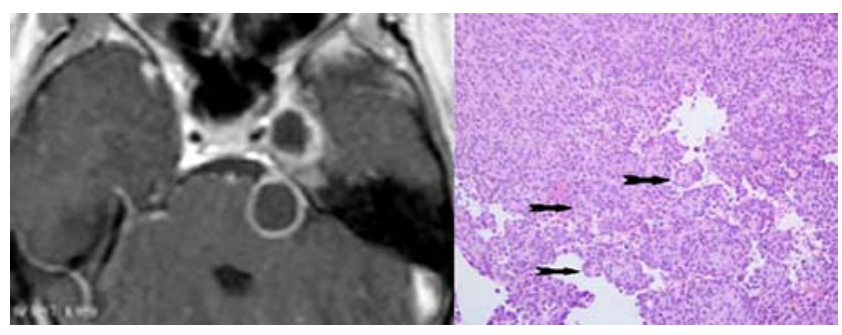

PO7:2

\section{IMAGING OF MELANOCYTIC MELANOCYTOMA}

M. Spampinato, C. Moses, C. Welsh, N. Besenski

Medical University of South Carolina, Charleston, USA

\section{PURPOSE}

Primary meningeal melanocytic tumors (PMMTs) are rare entities characterized by varied grades of malignancy. Our goal is to present the imaging findings and pathologic correlations of an uncommon case of melanocytoma of the craniocervical junction with diffuse spinal and intracranial leptomeningeal involvement. METHODS

We reviewed the clinical records of a 55 year-old male who was admitted in our institution for progressive lower extremity weakness and difficulty walking. An MRI of the brain, an MRI of the spine, and pathology records were also reviewed.

\section{RESULTS}

The MRI of the brain and of the spine demonstrated widespread leptomenigeal disease and intraventricular masses. The abnormal leptomeningeal and intraventricular tissue demonstrated increased signal on non-contrast T1-weighted images and enhancement after gadolinium administration. The MRI of the cervical spine demonstrated an intradural extramedullary enhancing mass enveloping the medulla and upper cervical cord with the bulk of tumor at the foramen magnum and at the C2-C3 level. At surgery the cervical spine mass was deemed unresectable and multiple biopsies were obtained. Histology demonstrated a melanocytic lesion consistent with melanocytoma. The patient was transferred to a rehabilitation facility and ultimately became paraplegic. CONCLUSION

PMMTs are rare entities that arise from melanocytes within the leptomeninges. The presence of extensive leptomeningeal and dural disease found in primary meningeal melanocytic tumors contrasts with the localized presentation of metastatic melanoma, despite a similar appearance on histology. The differential diagnosis includes drop metastases from pigmented medulloblastomas, ependymomas, or pineoblastomas. 


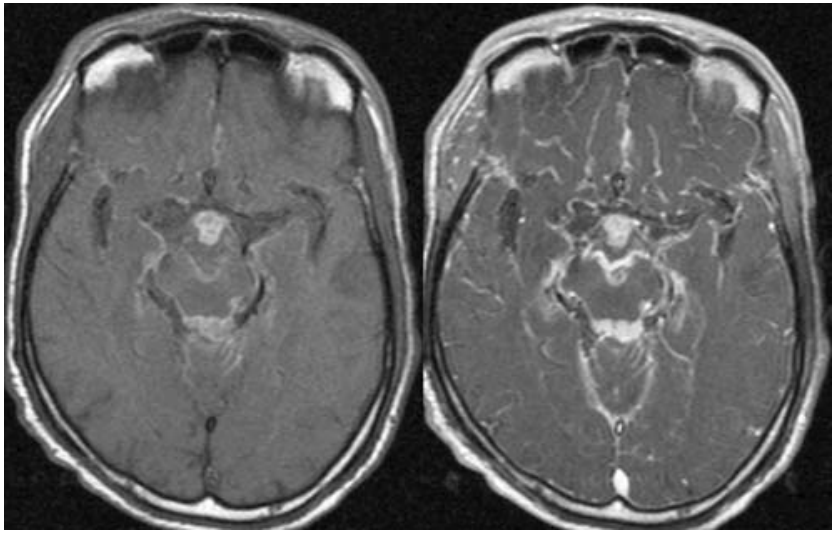

PO7:3

\section{PERCUTANEOUS FEMOROPLASTY IN PATIENT AFFECTED BY ADVANCED MULTIPLE MYELOMA WITH A PROXIMAL FEMORAL LOCALIZATION: A CASE REPORT}

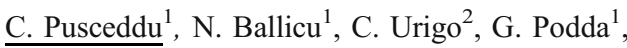
S. Profili ${ }^{2}$, G. Meloni ${ }^{2}$

${ }^{1}$ Oncology Hospital Businco, Cagliari, Italy; ${ }^{2}$ Department of Radiology University, Sassari, Italy; ${ }^{3}$ Oncology Hospital Cagliari Italy

\section{PURPOSE}

To evaluate the feasibility and safety of percutaneous femoroplasty under CT and fluoroscopic guidance for treatment of large osteolytic defect of the proximal femur, with prophylactic bonecement injection, in patient with refractory pain and high risk of fracture.

\section{MATERIALS AND METHODS}

A 61 year-old woman, affected by multiple myeloma with large osteolytic area in the head and nech of right femur with high risk of fracture, was treated with bone-cement injection. The patient was unsuitable candidate for surgery according to multidiscplinary consensus. Lesion approach was performed using a 10-gauge bone biopsy needle under CT and fluoroscopic guidance. Subsequnty, under fluoroscopic guidance $15 \mathrm{cc}$ of polymethylmetacrylate (PMMA) was injected into the lesion.

RESULTS

Technical success was achieved and no methylmetacrylate leakage was detected. Pain relief was complete within 1 week. No complication occurred after a two months follow-up.

CONCLUSIONS

Percutaneous femoroplasty is feasible and safe for the treatment of osteolityc lesions of femoral head and neck due to multiple myeloma and it prevents the pathological fracture.

\section{PO7:4}

\section{CT-GUIDED RADIOFREQUENCY (RF) ABLATION WITH BONE CEMENT INJECTION IN THE TREATMENT OF PELVIC AND FEMORAL METASTASES.}

\section{Pusceddu}

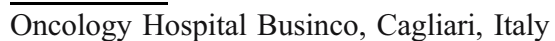

\section{PURPOSE}

The purpose of this study was to evaluate feasibility and safety of radiofrequency ablation with bone cement injection in the treatment of pelvic and femoral metastases.

METHODS AND MATERIALS

Betwen November 2004 and March 2007, 13 patients ( 2 man and 11 woman, mean age 65 years) with unresectable femoral $(n=3)$, sacral $(n=4)$, acetabular $(n=5)$ and ischial tuber $(n=1)$ osteolytic metastases were treated with percutaneous radiofrequency (RF) ablation and acrylic cement injection. All patients reported refrectory pain and deficiency of walking ability. Tumor diameter ranged from 3 to $8 \mathrm{~cm}$. For the ablation we used an expandable electrode catheter (LeVeen, Boston Scientific Corporation) in 9 cases and an Cool-Tip needle (Radionics, Burlington, MA, USA) in other 4 cases. After the needle electrode were inserted in the tumor with CT guidance, RF energy was applied. After completion of RF ablation a polymethyl-methacrylate (PMMA) solution were injected through the bone needle under continuous fluoroscopic guidance. Osteoplasty were performed using from 3 to 18 (average 7) $\mathrm{mL}$ of PMMA. Pain was assessed with the Visual Analogic Scale (VAS) before the therapy and on follow-up of an average of 12 months.

\section{RESULTS}

The procedures were technically successful in all patients. No serious complication occurred. After the treatment all patients reported immediate pain reduction (VAS score fron 7.4 to 1.2 ) and an improvement of walking ability.

\section{CONCLUSION}

Combined RF ablation with bone cement injection in the tratment of pelvic and femoral metastases is feasible and safe procedure. If confirmed by further investigations, this therapy may be considered a new therapeutic options for patients with unresectable pelvic and femoral metastases.

\section{PO7:5}

\section{THE MANY FACES OF CRANIAL CHORDOMAS}

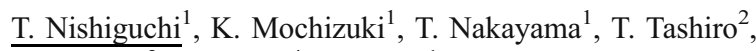
K. Wakasa ${ }^{3}$, K. Ohata ${ }^{4}$, Y. Inoue

${ }^{1}$ Department of Radiology, Osaka City University Graduate School of Medicine, Osaka, Japan; ${ }^{2}$ Department of Radiology, Yodogawa Christian Hospital, Osaka, Japan; ${ }^{3}$ Department of Pathology, Osaka City University Graduate School of Medicine, Osaka, Japan; ${ }^{4}$ Department of Neurosurgery, Osaka City University Graduate School of Medicine, Osaka, Japan 


\section{PURPOSE}

1. To review protean imaging manifestations of cranial chordomas.

2. To discuss the controversial entity of retroclival ecchordosis physaliphora (EP).

METHOD

Twenty-five pathologically proven chordomas and two EP in our institution (male to female: 13 to 14, ranging from 11 to 77 years old) from April 1994 to September 2006 were retrospectively reviewed and analyzed from a multifarious perspective. All cases were preoperatively evaluated by a 1.5 Tesla MR scanner and CT scanner.

\section{RESULT}

Twenty-two chordomas (including 18 classic chordomas and 4 chondroid chordomas) were intracranially located and three intradural chordomas were recognized. Two EP were noticed in the retroclival region. Among 22 intracranial chordomas, one occurred in the right cerebropontine angle, four arose from the petrous bone, one in the nasopharynx, and one in the occipitocervical junction. One clival chordoma demonstrated the subarachnoid dissemination at the level of the thoracic spine after the proton beam radiotherapy. Two out of three intradural chordomas showed intratumoral hemorrhage and a dural hole was confirmed in one case during surgery. Intrapontine large cyst formation with significant mass effect to the brain stem was noted in 2 retroclival chordomas. Two retroclival dormant lesions were deemed as EP and demonstrated no contrast enhancement on MR imaging. CONCLUSION

Imaging findings of cranial chordomas vary and may be misleading. Awareness of these protean imaging manifestations of cranial chordomas would be beneficial to radiologists.

\section{PO7:6}

\section{PERICALLOSAL LIPOMA BY 3.0-T MAGNETIC RESONANCE}

J. Rodríguez, T. Moreno, J. Onofre

Hospital Christus Muguerza Alta especialidad, Monterrey, Mexico

\section{OBJECTIVE}

Describe the imaging characteristics of a pericallosal lipoma using 3Tesla Magnetic Resonance (MR).

MATERIALS AND METHODS

We report a young girl with a pericallosal lipoma found incidentally during neuroradiological screening, including computed tomography (CT) and 3.0-Tesla MR, its associated malformation and literature review.

RESULTS

CT of the head revealed a large hypodense pericallosal lesion with - $70 \mathrm{HU}$ and short T1 and T2 on MR, associated with hypoplasia of the corpus callosum. Conclusion: This case showed the typical 3Tesla-MR characteristics of pericallosal lipoma. Intracranial lipomas are generally asymptomatic and require no therapy.

\section{PO7:7}

MR APPERANCE OF DEMYELINATION PATTERN
ASSOCIATED WITH PRIMARY LYMPHOMA
OF THE CENTRAL NERVOUS SYSTEM.
CORRELATION WITH HISTOPATHOLOGIC
FEATURES.

A. Mas bonet ${ }^{1}$, M. Picado ${ }^{1}$, A. Gene ${ }^{2}$, M. Couce Matovelle ${ }^{2}$, M. Brell $^{3}$, S. Miralbes Celma ${ }^{1}$, A. Moll Servera ${ }^{1}$

${ }^{1}$ Department Neuroradiology. Hospital Son Dureta, Palma de Mallorca, Spain; ${ }^{2}$ Department Pathology. Hospital Son Dureta, Palma de Mallorca, Spain; ${ }^{3}$ Department Neurosurgery. Hospital Son Dureta, Palma de Mallorca, Spain

\section{PURPOSE}

Our purpose is to describe the MR apperance of demyelination pattern associated with primary central nervous system (CNS) lymphoma in correlation with histopathologic features.

METHODS

We performed a retrospective review of MR studies on 15 patients with histopathologic proven CNS lymphoma, 4 of these had demyelination. We compare the imaging findings of these 4 with proven demyelination with the imaging findings of the others eleven. RESULTS

A total of 15 patients , 9 men (7 HIV+) and 6 women (non $\mathrm{HIV}+$ ) with proven primary CNS lymphoma were examined with MRI before byopsy. Two women (non HIV+) and two men (HIV+) had a demyelination pattern in the histopathologic findings.

CONCLUSION

Very few reports have been published to relate the demyelination with primary CNS lymphoma. In the MRI before byopsy it difficult to distinguish between perilesional vasogenic brain edema and demyelination, but some ancillary signs can orient us to this findings.

\section{PO7:8}

\section{RADIATION INDUCED NEUROFIBROSARCOMA OF CERVICAL SPINE. AN INTERESTING CASE.}

S. Lyra $^{1}$, K. Bakopoulos ${ }^{2}$, A. Papatheodorou ${ }^{1}$, A. Kokkinaki ${ }^{1}$, E. Samaras2, P. Ellinas ${ }^{1}$, V. Samaras ${ }^{3}$, V. Varsos ${ }^{2}$

${ }^{1}$ Radiology Department Red Cross Hospital, Athens, Greece; ${ }^{2}$ Neurosurgery Department Red Cross Hospital, Athens, Greece;

${ }^{3}$ Pathology Department Red Cross Hospital, Athens, Greece

\section{PURPOSE}

Neurofibrosarcoma as a late result of irradiation is an uncommon although possible finding (more often in patients with neurofibromatosis I). We present the case of a young female who developed neurofibrosarcoma 14 years after radiation therapy for acute lymphogenic leukemia (ALL).

METHODS

A 19 year old female was admitted to our Magnetic Resonance (MR) department for evaluation of acute onset of tetraplegia. She 
had been treated for ALL when she was 5 years old and the treatment included marrow transplantation chemotherapy and irradiation therapy. No family history of neurofibromatosis was mentioned.

The patient was submitted to MR examination of the spine. The examination protocol included T2 turbo spin echo weighted images (T2TSEWI), short time inversion recovery (STIR), T2* gradient echo (GRE) as well as T1TSEWI before and after intra venous (IV) administration of Gadolinium (Gd).

\section{RESULTS}

The MR examination revealed a strongly enhancing, intradural, extramedullary mass at the lower cervical spine (A6-T1) compressing the spinal cord. The lesion had a dumb bell appearance extending through the enlarged A6-A7 and A7-A8 right intervertebral foramen to the supraclavicular region. The radiological differential diagnosis included: neurofibroma, malignant neurofibroma and neurofibrosarcoma. The patient was referred for operation and the biopsy revealed neurofibrosarcoma. CONCLUSIONS

Neurofibrosarcoma should be included in the differential diagnosis of intradural, extramedullary spine lesions, as a late consequence in patients subjected to bone marrow irradiation.

\section{PO7:9}

\section{CONTRAST ENHANCED MR IMAGING OF METASTATIC BRAIN TUMOR AT 3 TESLA}

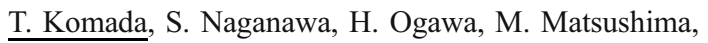
S. Kubota, H. Kawai, H. Fukatsu

Nagoya university, Nagoya, Japan

The objective of this study was to evaluate the newly developed whole-brain isotropic 3D T1-weighted turbo spine echo imaging with variable flip angle echo train (3D-SPACE) for contrast enhanced T1-weighted imaging in detecting brain metastases at 3 Tesla ( $\mathrm{T})$. Twenty patients with suspected brain metastases underwent postcontrast study with 3D-SPACE, magnetizationprepared rapid gradient-echo (MP-RAGE) and 2D T1-weighted spine echo (SE) images at 3 T. 3D-SPACE, MP-RAGE and 2D SE images were compared qualitatively by using signal-to-noise ratio (SNR) for gray matter (GM) and white matter (WM), and contrastto-noise ratio (CNR) for GM-to-WM, lesion-to-GM and lesion-toWM. Two blinded radiologists evaluated the detection of brain metastases by segment-by-segment analysis and the continuouslydistributed test. CNR between GM and WM was significantly higher on the MP-RAGE images than on 3D-SPACE images (P $<.01)$. There were not significant differences in each sequence in detection of brain metastases by segment-by-segment analysis and the continuously-distributed test. However, in some cases, the lesions in 3D-SPACE images were easier to detect than in other sequences, and the vascular signals in MP-RAGE and 2D-SE images mimicked like lesions, those could be suppressed in 3DSPACE images. In detection of brain metastases at $3 \mathrm{~T}$ MRI, 3DSPACE imaging provides an effective, alternative approach to MP-RAGE imaging for 3D T1-weighted imaging.

\section{PO7:10}

\section{CRANIAL HEMANGIOBLASTOMAS IN VON HIPPEL-LINDAU DISEASE}

$\underline{\text { C. Hader }}^{1}$, J. Spreer ${ }^{1}$, S. Gläsker ${ }^{2}$, H. Neumann ${ }^{3}$

${ }^{1}$ Department of Neuroradiology, Albert-Ludwigs-Universität, Freiburg, Germany; ${ }^{2}$ Department of Neurosurgery, Albert-LudwigsUniversität, Freiburg, Germany; ${ }^{3}$ Department of Nephrology and Hypertension, Albert-Ludwigs-Universität, Freiburg, Germany

\section{PURPOSE}

Cranial hemangioblastomas are one of the main manifestations in von Hippel-Lindau disease. Because of their space occupying character they can cause neurological symptoms and an operative resection of the tumors can be necessary. The aim of the study was to evaluate the prevalence, size and frequency of surgery of cranial hemangioblastomas in the Freiburger group of von Hippel-Lindau patients.

\section{METHODS}

Serial magnetic resonance images (MRI) of 226 patients with clinically or genetically proven von Hippel-Lindau disease were evaluated retrospectively. The location (cerebellum, brainstem or supratentorial) and type (solid or cystic) of the tumors were registered and the size of the tumors and their associated pseudocysts were measured. In addition, it was noted whether the tumor was operated or controlled by follow up MRI.

RESULTS

103 of 226 patients showed cranial hemangioblastomas (46\%). Altogether we found 455 tumors (cerebellum 85\%, brainstem $12 \%$, supratentorial $3 \%$ ). The diameter of the tumors ranged from 2 to $49 \mathrm{~mm}$. The majority of the tumors (79\%) was less than $10 \mathrm{~mm}$ in diameter. 126 of 455 tumors were operated (28\%). $12 \%$ of the tumors showed associated pseudocysts. Cystic tumors were significantly larger than solid tumors (cystic $29 \mathrm{~mm}$, solid $8 \mathrm{~mm}$, $\mathrm{p}<=0,000001)$ and were operated more often. All cystic tumors showed an increase in size during the follow up, whereas the overall rate of tumor progress was $44 \%$.

CONCLUSION

Cranial hemangioblastomas are a common manifestation in von Hippel-Lindau disease but in our patient group only $28 \%$ of all tumors needed an operative treatment. Tumor associated pseudocysts occurred in $12 \%$ of all tumors. All cystic tumors showed tumor progress during the follow up. They were larger and needed more often surgery than solid tumors. These facts should be considered in the management of patients with von Hippel-Lindau disease.

\section{PO7:11}

\section{A RARE CASE OF INTRADURAL INTRAMEDULLARY MEDULLOEPITHELIOMA}

A. Elias, P. Robertson, C. Fox, N. Patel, K. Muraszko, P. Maly-Sundgren

${ }^{1}$ University of Michigan, Ann Arbor, USA 


\section{PURPOSE}

The purpose is to describe and illustrate a rare case of intraspinal medulloepithelioma. Medulloepithelioma is a rare neuroectodermal tumor of the central nervous system, primitive in nature with a remarkable potential to differentiate. Histologically, it is very similar to primitive neuroectodermal tumors (PNET). This tumor responds to radiotherapy and the otherwise poor prognosis increases significantly with total excision.

\section{PATIENT AND METHODS}

A 2-year-old, otherwise healthy boy, with history of worsening back pain and abnormal posturing since a fall 2 months prior admission. A brain and total spine MRI revealed a heterogeneous enhancing mass within the spinal canal extending from T10 to the L5 level. The mass appeared to arise partially from the conus medullaris with exophytic growth and intradural extramedullary extension.

A T11 to S1 laminoplasty was performed with complete resection of an intradural, intramedullary spinal tumor and micro dissection of the spinal cord (filum terminale). During postoperative radiotherapy the patient had interval development of a recurrent intradural extramedullary enhancing tumor at the level of the conus medullaris. Chemotherapy was then initiated with cisplatinum, cyclophosphamide, etoposide, and vincristine. The patient is presently 3 month out in near complete regression.

\section{RESULTS}

Pathologic examination revealed tubular neoplastic neuroepithelium that mimic the embryonic neural tube and pseudostratified epithelium with mitotic cells located toward the luminal surface consistent with medulloepithelioma.

\section{CONCLUSION}

Two-year-old male with complete resection of a spinal medulloepithelioma and postoperative radiation therapy who had tumor recurrence and has responded with disease free survival after treatment with cisplatinum, cyclophosphamide, etoposide, and vincristine.

\section{PO7:12}

\section{CLINICAL PRESENTATION AND IMAGING FEATURES OF INTRADURAL SPINAL TUMORS}

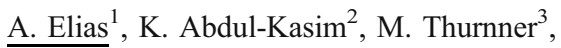
P. Maly-Sundgren ${ }^{1}$, P. Mckeever ${ }^{1}$

${ }^{1}$ University of Michigan, Ann Arbor, USA; ${ }^{2}$ University of Lund, Malmö University Hospital, Lund, Sweden; ${ }^{3}$ Medizinische Universität Wien, Vienna, Austria

\section{PURPOSE}

The differential diagnosis of intradural spinal tumors is primarily based on location, but clinical presentation, age, and gender plays an integral part determining the diagnosis. The purpose of this exhibit is to describe and illustrate the MRI imaging features of the more common intradural extra and intramedullary spine lesions. METHODS

The most important imaging tool for evaluating the intradural spinal is magnetic resonance imaging (MRI). MRI has the capability to classify spinal tumors into extradural, intraduralextramedullary or intradural intramedullary lesions. CT or plain radiographs might play a role in the evaluation of the surrounding bony structures.

RESULTS

This presentation will illustrate and describe the characteristics of the more common intradural spinal tumors. Clinical presentation, characteristic imaging features of different intradural spinal cord tumors will be presented and differential diagnosis by location, age and imaging findings will be discussed. Each tumor will illustrated with relevant CT and MR images. All here presented cases are histopathologically proven.

\section{CONCLUSION}

The current classification and imaging features of the most common spinal cord and intradural tumors is the focus of this exhibit. It is important to recall that for the evaluation of the bony spine, both computer tomography (CT) and MRI are essential imaging tools for diagnosis. The use of the current comprehensive classification of spinal tumors by the World Health Organization (WHO) is illustrated.

\section{PO7:13}

\section{PITUITARY MACROADENOMA PRESENTING WITH PITUITARY APOPLEXY ASSOCIATED WITH VASOSPASM AND CEREBRAL ISCHEMIA}

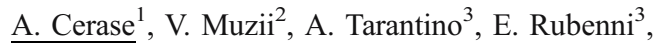
A. Lapi $^{4}$, G. Signori ${ }^{2}$, L. Palma ${ }^{2}$, C. Venturi ${ }^{1}$

${ }^{1}$ UOC Neuroradiologia, Policlinico Santa Maria alle Scotte, Siena Italy; ${ }^{2}$ UOC Neurochirurgia, Università, Siena Italy; ${ }^{3}$ Istituto di Radiologia, Università, Siena Italy; ${ }^{4}$ UOC Medicina Interna, Policlinico Santa Maria alle Scotte, Siena Italy

Pituitary apoplexy (PA) may present catastrophically, frequently mimics other intracranial pathologies, and generally results from infarction or hemorrhage of pre-existing pituitary adenoma. Other risk factors include trauma, increased intracranial pressure, anticoagulation, bromocriptine therapy, diabetic ketoacidosis, conventional angiography, irradiation, and open heart surgery, intraoperative manipulation, dehydration following hyperthermia, cerebral salt wasting syndrome. Necessity and urgency of macroadenoma surgical decompression depends on presence, rate, and changes of visual symptoms. Conservative management is possible, with tumor ischemia/infarction resulting in mass regression. Rarely, PA is complicated by cerebral ischemia, whose etiology is difficult to determine. Stenosis or occlusion of major cerebral arteries may be caused by vasospasm due to subarachnoid blood released from PA, or vasoactive agents released from tumor or hypothalamohypophyseal area, and from tumor mass effect itself. The purpose of this scientific exhibit is to discuss these issues by reporting clinical and neuroradiological findings in two male patients with unknown non-secretory macroadenoma presenting with PA, and vasospasm resulting in cerebral ischemia. One had severe headache, and visual deficits, the other was found on home floor, unable to get up, conscious, dysarthric, with left ptosis and 
looked dehydrated. In both patients, CT and MRI showed a hemorrhagic macroadenoma. In one, acute subarachnoid hemorrhage ( $\mathrm{SAH})$ was associated, in the other lumbar puncture ruled out SAH. Both patients had vasospasm, detected by either DSA or MRA, resulting in ischemia. One patient underwent macroadenoma total resection, the other is followed-up without surgical resection, given absence of significant visual deficit, gradually clinical recovery, and control MRI/MRA showing moderate macroadenoma regression, and vasospasm resolution.

Cerebral ischemia and infarction following PA is rare, but should be suspected even in the absence of SAH. MRI is the exam of choice to detect PA, vasospasm by MRA, and early changes of brain ischemia. DWI is useful in detecting macroadenoma ischemic changes.

\section{P07:14}

\section{INTRACRANIAL INVOLVEMENT \\ IN PLASMACYTOMAS AND MULTIPLE MYELOMA. A PICTORIAL ESSAY}

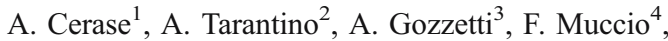
$\overline{\text { I. Vallone }}^{1}$, G. Esposito ${ }^{4}$, A. Di Blasi ${ }^{5}$, C. Venturi ${ }^{1}$

${ }^{1}$ UOC Neuroradiologia, Policlinico Santa Maria alle Scotte, Siena, Italy; ${ }^{2}$ Istituto di Radiologia, Università, Siena, Italy; ${ }^{3}$ Istituto di Ematologia, Università, Siena, Italy; ${ }^{4}$ UOC Neuroradiologia, Azienda Ospedaliera G.Rummo, Benevento, Italy; ${ }^{5} \mathrm{UOC}$ Anatomia Patologica, Azienda Ospedaliera G. Rummo, Benevento, Italy

Plasmacytomas and multiple myeloma (MM) with intracranial growth are rare. The purpose of this pictorial essay is to increase awareness to their neuroradiological findings by reporting CT and MRI findings in seven patients (4 females, and 3 males, age range: 62-82 years) with plasmacytoma (n: 2 , one from cranial vault, one from zygomaticosphenotemporal buttress) and MM (n: 5) with intracranial growth. In a plasmacytoma MRI included DWI, PWI, and ${ }^{1} \mathrm{H}$ multivoxel MRS, in another one DSA was performed. Three patients underwent surgery. Clinical and neuroradiological follow-up ranges from one week to 3.5 years. One patient with MM had leptomeningeal disease. A condylar occipital bone lesion, and a cavernous sinus region dural lesion were disease presenting sign in two patients.

Most commonly, CT and MRI showed well-defined destructive masses arising from osseous structures. Cranial bone lesions were not seen in primary meningeal disease. The mass generally appears hyperdense at unenhanced $\mathrm{CT}$, iso- to hyperintense on T1w, and iso- to hypointense on T2w MR images, with mild to marked contrast-enhancement possibly heterogeneous. MRI provides the best assessment of intracranial extent. At DWI, PWI, and ${ }^{1} \mathrm{H}$ multivoxel MRS, one plasmacytoma showed restricted diffusion, increased $\mathrm{rCBV}$, elevated Cho, reduced NAA and $\mathrm{Cr}$. At angiography, plasmacytomas generally show a blush supplied by meningeal arteries.

Neuroradiology generally lack of specificity, since findings may be no different from meningioma, plasma cells granuloma, metastasis, lymphoma, dural sarcoma, infectious meningitis, and leptomeningeal carcinomatosis. Diagnosis is usually made when lesions appear in the context of a previously diagnosed MM or after finding criteria for MM. Surgery is indicated in selected cases. Cerebrospinal fluid examination remains definitive test for diagnosing leptomeningeal myelomatosis, however in daily clinical practice it is reserved for selected cases.

Intracranial plasmacytomas and MM must be taken in account in differential diagnosis of cranial osseous and meningeal disease.

\section{PO7:15}

\section{CHIASMAL SYNDROME : A PICTORIAL REVIEW OF MRI FEATURES}

A. Camins, C. Majós, S. Castañer, J. Sánchez, M. Cos, E. Alonso, C. Aguilera Institut de Diagnòstic per la Imatge. Ciutat Sanitaria i Universitària de Bellvitge. Hospital Duran i Reynals, Barcelona, Spain

\section{PURPOSE}

To review the normal anatomy of the visual system with emphasis in the optic chiasm.

To illustrate the MR imaging features of the patients with Chiasmal syndrome, such as neoplasms, infections, congenital malformations, and other miscellaneous conditions.

To evaluate the correlation between neurologic manifestations and MR imaging findings.

METHODS AND RESULTS

Chiasmal syndrome is the constellation of signs and symptoms that are associated with lesions of the optic chiasm. Visual loss is often the initial manifestation of disorders involving the optic chiasm. Hemianopic defects and preferential involvement of the temporal visual field are the earliest and most common visual deficits. Most chiasmal lesions can be categorized as intrinsic, with the lesion involving the substance of the optic chiasm itself, or extrinsic, due to mechanical compression from adjacent structures.

The standard imaging protocol for evaluation of chiasmatic lesions includes 3-mm coronal STIR, 3-mm axial and sagittal T1-WI, and after administration of gadolinium, 3-mm axial fat-saturation T1-WI and coronal non-fat-saturation T1-WI. Sagittal T2-WI, axial FLAIR, DWI and spectroscopy were used optionally. The extensive spectrum of pathologies that can affect this anatomical region have been grouped in relation to their origin (intrinsic or extrinsic) and etiology: infectious and inflammatory (sarcoidosis, tuberculosis), tumoral (pituitary tumour, meningioma, glioma, chordoma, craniopharyngioma, lymphoma), vascular (aneurysm, cavernous haemangioma), congenital (Rathke cleft cyst) and iatrogenic.

\section{CONCLUSION}

Magnetic resonance imaging is the best mode of neuroimaging for most chiasmal lesions and may also provide clues to the etiology of an isolated chiasmal syndrome. 


\section{PO7:16}

\section{MRI AND CT FINDINGS OF GIANT CELL TUMOR OF THE SPHENOID BONE: A CASE REPORT AND REVIEW OF THE LITERATURE}

V. Calistri ${ }^{1}$, E. Venditti ${ }^{1}$, V. Galasso ${ }^{1}$, M. Piattella ${ }^{1}$,

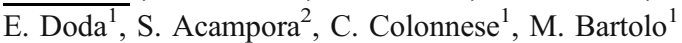
${ }^{1}$ Neuroradiology Department Irccs Neuromed, Pozzilli (IS), Italy; ${ }^{2}$ Neurosurgery Department Irccs Neuromed, Pozzilli (Is), Italy

\section{PURPOSE}

giant cell tumor of the sphenoid bone is extremely rare. This tumor is rarely located in the cranial vault. We investigated CT and MR findings of a case of giant cell tumor of the sphenoid bone in a 19 years old patient treated with surgery.

\section{METHODS}

a 19 years old girl was admitted at our hospital for a progressive onset of headache unresponsive to NSAIDs that was accompanied by diplopia and pain in left eye. She was then submitted to a brain MRI that revealed an extensive osteolytic mass localized within the sphenoid body with extension to the clivus, the ethmoid and nasal cavities, and the cavernous sinus bilaterally.

RESULTS

The patient underwent a transsphenoidal biopsy of the mass. A giant cell tumor was diagnosed. She was then submitted to surgical treatment of the mass and subsequent MRI and CT scans revealed a subtotal resection of the tumor.

CONCLUSIONS

Giant cell tumors of sphenoid bone are amongst the most rare of cranial-based tumors. It is extremely important to differentiate this tumor from other pathologies that are located in the parasellar region. CT and MRI allowed a exhaustive evaluation of morphology and extension of the bone mass and its relation with the surrounding structures. Furthermore they yield a valid tool that not only support histological results but also provides fundamental information for surgical and radiation therapy.

\section{PO7:17}

\section{TRANSIENT CT CORTICAL ENHANCEMENT INDUCED BY CHEMOTHERAPY IN A PATIENT WITH RENAL MALIGNANT TUMOR}

\author{
A. Bacci ${ }^{1}$, P. Ambrosetto ${ }^{2}$, M. Maffei ${ }^{1}$, G. Cavallo ${ }^{3}$, \\ R. Agati ${ }^{1}$, M. Leonardi ${ }^{1,2}$ \\ ${ }^{1}$ Neuroradiology Department Bellaria Hospital, Bologna, \\ Italy; ${ }^{2}$ Neuroradiology Department, University of Bologna, \\ Bologna, Italy; ${ }^{3}$ Oncology Department Bellaria Hospital, \\ Bologna, Italy
}

We report a case of a patient treated by chemotherapy for a renal cancer, who presented diffuse, reversible, bilateral enhancing cortical lesions, detected by $\mathrm{CT}$.
This 60-year-old white man underwent radical left nephrectomia 14 years prior to admission to the Bellaria Hospital due to a renal cancer and partial right nephrectomia for the same cancer 13 years after. Postoperatively he was treated by $5 \mathrm{FU}$ and Interleukin 2 therapy. 10 months later, during the chemotherapy, the patient had been admitted to our Oncological Departmen for a syncopal seizure. The neurological evaluation showed a mild walking impairment with no additional deficits.

CT revealed gyral pattern enhacement lesions in the cerebral and cerebellar cortex, with no vascular distribution, edema nor mass effect. Cerebrospinal fluid was negative. Laboratory data were uncontributory. MR performed 4 days later detected only small hyperintense $\mathrm{T} 2$ areas in the supratentorial white matter and no cortical enhancement after contrast medium administration. Repeated CT scan 9 days later was absolutely normal.

According to our review of the literature, we speculate that these reversible cortical lesions, mimicking those found in the cyclosporine encephalopathy and in the reversible posterior hypertensive encephalopathy, could be due to the chemotherapy 's neurotoxic effect.

Therefore in our case we think that the reversible enhancing cortical lesions can be due to a direct chemotherapy's neurotoxic effect, or can be produced by a possibly missed transient episode of hypertension and not by a metastatic tumor. A localized breakdown of the blood-brain barrier with increased permeability and/or hyperperfusion, could explain these radiological findings. In conclusion we alert Neuroradiologist to be aware of this unusual situation, and emphasize the usefulness of early repeated CT and/or MRI examinations, when diffuse cortical enhancing areas are detected in patient undergone chemotherapy for malignant tumors.

\section{PO7:18}

CLINICAL APPLICATION OF FIBER-TRACKING: NEOPLASTIC PATHOLOGY

\author{
$\underline{\text { S. Battaglia }}^{1}$, D. Cevolani ${ }^{1}$, R. Agati ${ }^{1}$, V. Piscitelli ${ }^{2}$, \\ M. Leonardi ${ }^{1}$ \\ ${ }^{1}$ Neuroradiology Department, Bologna, Italy; ${ }^{2}$ Federico Ii Univer- \\ sity, Naples, Italy
}

The goal of surgical treatment of the brain tumors located in eloquent areas is to maximize the tumor resection while minimizing the damage to cortical and subcortical functions of the intact brain.

To preserve brain functions it is important to identify the eloquent cortex (using fMRI, for example) but also the white matter tracts. Diffusion tensor imaging (DTI) study allows to identify white matter tracts, because the possibility to determine the preferential direction of water molecules movement in cerebral tissue, which is strongly directed by the tissue anisotropy.

The acquired informations can be represented through bidimensional parametric maps (e.g. fractional anisotropy, and colour coded) and in a three-dimensional form through the "FiberTracking" techniques. 
In our institution we have recently performed DTI studies for surgical planning using a $3 \mathrm{~T}$ MR system (Signa Excite, General Electric, Milwakee, WI), combined with fMRI studies.

Our aim was to evaluate the usefullness of the acquired informations in view of a possible integration in a neuronavigation system.

Regarding neoplastic pathology, tractography may differentiate: displacement of fibres (qualitative hemispheric asymmetry) and infiltration/destruction of fibres (quantitative hemispheric asymmetry).

However there is an important limit of this method, in presence of perilesional oedema: the marked reduction of Fractional Anisotropy due to oedema may lead to errors in fiber-tracking with a non accurate estimation of the size of the tracts passing through the oedematous areas.

In our experience tractography is able to show the spatial relationship between tumour and the adjacent white matter tracts (in particular pyramidal tract) to maximize the surgical resection preserving critical anatomical structures such as eloquent cortical areas and pyramidal tract, adjacent to brain tumors. Furthermore, tractography could contribute to shortening the time required for surgical procedure when evoked cortical and sub-cortical potentials (ECP) are used.

In conclusion tractography, and more in general DTI, could provide useful information for preoperative and intraoperative planning.

\section{PO7:19}

\section{CHILDHOOD SPINAL GLIOBLASTOMA MULTIFORME WITH INTRACRANIAL DISSEMINATION.}

$\underline{\text { S. Battaglia }}^{1}$, L. Albini Riccioli ${ }^{1}$, F. Bartiromo ${ }^{3}$, $\overline{\text { E. Galassi }^{2}}$, A. Marliani ${ }^{1}$, M. Leonardi ${ }^{1}$

${ }^{1}$ Neuroradiology Department Bellaria Hospital, Bologna, Italy; ${ }^{2}$ Neurosurgery, Bellaria Hospital, Bologna, Italy; ${ }^{3}$ Federicoii University, Naples, Italy

\section{INTRODUCTION}

Primary spinal cord tumours in children is an uncommon entity and metastasis are extremely rare.

Most childhood intramedullary tumours are astrocytomas (47$88 \%$ ), followed by ependymomas (24-37\%) Spinal cord glioblastoma in children is only rarely been reported.

It most involves thoracic region with a predilection for the second and third decade of life. We report a case of thoracic glioblastoma multiforme with intracranial dissemination in early stage of clinical course

\section{CASE REPORT}

An 11 year old boy, on admission at emergency room, presented with a progressive and rapid paraparesis and tactile hypoesthesia. A magnetic resonance imaging (MRI) scan of spinal cord revealed an intramedullary and exophytic mass extending from Th4 to Th5; the lesion showed an inhomogeneous contrast enhancement after gadolinium injection. Full neuraxis MRI showed no other lesions. The patient underwent laminotomy and tumour excision; the histological diagnosis was glioblastoma multiforme. Postoperative course was uneventful.

Postoperative radiotherapy and chemotherapy were administered, but 3 months later the patient presented symptoms of raised intracranial pressure.

MRI was performed and showed hydrocephalus, due to leptomeningeal dissemination, as documented by diffuse enhancement of cerebral and spinal subarachnoid spaces. Left ippocampus was also involved

A CSF ventriculo-peritoneal shunt was inserted with resolution of intracranial hypertension

The patient deteriorated progressively and died 6 month after the onset of symptoms.

\section{CONCLUSIONS}

Glioblastoma multiforme of spinal cord is a highly aggressive, quite rare entity; in most of the patients the prognosis remains poor despite aggressive surgical and oncological treatment.

\section{PO7:20}

\section{A LOW GRADE TUMOR MIMICKING INFARCTION ON CT PERFUSION}

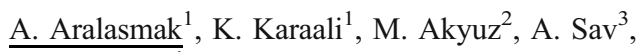 \\ C. Ozkaynak ${ }^{1}$ \\ ${ }^{1}$ Akdeniz University, Department of Radiology, Antalya, Turkey; \\ ${ }^{2}$ Akdeniz University, Department of Neurosurgery, Antalya, \\ Turkey; ${ }^{3}$ Marmara University, Department of Pathology, Istanbul, \\ Turkey
}

\section{PURPOSE}

Extraventricular neurocytoma is a very rarely seen low grade tumor. We presented CT perfusion (CTP) findings of a pathologically proven extraventricular neurocytoma, which were mimicking infarction.

\section{METHODS}

A 45 year old male patient was referred for stroke like symptoms of his left extremity, worse in the last days. CT, CTP and contrast enhanced MRI were performed. On CTP following a bolus contrast of $50 \mathrm{cc}$, sequential images were obtained through the lesion at four slice locations with each of $8 \mathrm{~mm}$ in thickness. Thereafter, perfusion maps (CBV, CBF, MTT) were generated. Permeability was not mapped.

RESULTS

On MRI, there was a peripherally located intraaxial mass lesion with no enhancement. On CTP, the lesion revealed prolonged MTT with decreased $\mathrm{rCBV}$ and $\mathrm{rCBF}$, consistent with infarction. Following 2 weeks, MRI was repeated with no change, suggestive of a tumoral mass. Surgical excision was performed with pathology of extraventricular neurocytoma.

\section{CONCLUSION}

Infarction is characterized by prolonged MTT and decreased $\mathrm{rCBF}$ and $\mathrm{rCBV}$. In tumors, $\mathrm{rCBV}$ and $\mathrm{rCBF}$ are very close to normal brain tissue, in high-grade tumors, values that are greater than normal. In neurooncology, rCBV and permeability maps are used for identification of the biopsy site and tumor grading. CTP of this 
extraventricular neurocytoma was unique because of prolonged MTT and decreased $\mathrm{rCBV}$ and $\mathrm{rCBF}$, which are similar to that seen in infarction zones.

\section{Trauma}

\section{PO8:1}

\section{DIFFUSION TENSOR MRI IN SEVERE DIFFUSE AXONAL} INJURY

N. Zakharova, V. Kornienko, A. Potapov, I. Pronin,

L. Fadeeva, K. Gorshkov, S. Takush

Burdenko Neurosurgery Institute, Moscow, Russia

\section{PURPOSE}

to study MR diffusion tensor imaging (DTI) and fiber tracking in patients (pts) with severe diffuse axonal injury (DAI).

\section{METHODS}

14 pts with DAI caused by severe head injury (Glasgow Coma score of 8 or less) were examined by conventional MRI (T1, T2, FLAIR) and DTI between 3-17 days after traffic accident. Outcome was evaluated by Glasgow Outcome Scale; 7 pts with moderate or severe disability had different level of hemiparesis; 3 pts in the vegetative state had tetraparesis. Average diffusion coefficient (DCav) and fractional anisotropy (FA) in five regions of interest (ROIs) in the genu and splenium of corpus callosum (CC), posterior limb of the internal capsule, midbrain and pons were measured. The same parameters were performed in the same ROIs in 8 healthy volunteers.

RESULTS

FA values in ROIs in the contralateral corticospinal tract (CST) in pts with hemiparesis significantly differed from the analogous values in the homolateral side and parameters obtained in volunteers. There was significant correlation between the level of hemiparesis and FA values in the posterior limb of the internal capsule at the contralateral side. DCav values in these structures outside the oedema and haemorrhage region did not significantly differ. 3 pts in the vegetative state and with tetraparesis showed significantly lower FA values in the bilateral CST $(\mathrm{p}<0,01)$ compared to those of volunteers. In all pts average FA and DCav values in splenium of $\mathrm{CC}$ were lower than in the volunteers $(\mathrm{p}<$ $0,05)$. Conventional MRI revealed no marked changes in genu of $\mathrm{CC}$ in 13 of the 14 patients. Average FA values in this group were significantly lower $(p<0,01)$ than in the volunteers. 3D reconstructions of CSTs in pts with severe hemiparesis revealed their asymmetry.

\section{CONCLUSION}

Diffusion-tensor MRI with FA evaluation and fiber tracking provides additional information about brain damage to conventional MRI.

\section{PO8:2}

\section{AIR IN THE SELLAR AND PARASELLAR REGION FOLLOWING ORBITAL INJURY WITH COMPRESSED AIR DEVICE}

S. Reimão, C. Morgado, L. Neto, J. Campos

Neurologic Imaging Department - Saint Mary's Hospital, Lisbon, Portugal

\section{PURPOSE}

Air collections in the sellar region are an uncommon finding that has been most frequently described following trauma with skull base fractures and as a complication of endonasal transsphenoidal surgery. We report the case of epidural pneumocephalus in sellar and parasellar region following orbital injury with compressed air hose. In our research we could not find reports of sellar air collections following trauma without associated skull or facial fractures.

CASE STUDY

A 29 years old male had an accident with a compressed air hose; while storing it, he accidentally pointed to the eye causing direct air trauma to the orbit resulting in severe pain, extensive subcutaneous and orbital emphysema with exophthalmia and limitation of eye movements. Brain and orbital CT showed extensive air collections in subcutaneous tissues and orbital compartment (intra and extra conical). Air extended to the orbital apex, cavernous sinus and sellar region extending superiorly to the sphenoidal jugum without any associated fracture. He received only symptomatic therapy.

\section{RESULTS}

The air collections progressively reabsorbed over time with significant clinical improvement.

\section{CONCLUSION}

Trauma to the orbit with high pressure air resulted in extensive local tissue damage and high velocity air extended through tissue plans not causing visible bone defects. Intracranial air appears to be in the epidural compartment, dissecting tissue plans following the superior orbital fissure till reaching the sellar region.

\section{P08:3}

\section{TRAUMATIC RETROCLIVAL HAEMATOMA IN AN ADULT: A CASE REPORT}

M. Michelassi ${ }^{1}$, M. Cosottini ${ }^{2}$, V. Ravelli ${ }^{2}$, S. De cori ${ }^{3}$, P. Carpeggiani $^{1}$, G. Parenti ${ }^{2}$

${ }^{1}$ AOU Pisa, Pisa, Italy; ${ }^{2}$ Department of Neuroscience, Pisa, Italy; ${ }^{3}$ Department of Radiology, Pisa, Italy

Post-traumatic retroclival epidural haematoma (REH) is an extremely rare event and only a few cases are described in literature. Most reported cases involve pediatric patients and are associated with a cervical spinal trauma. We described a case of a post-traumatic REH occurs in an adult. 
A 43-year-old female was admitted to our hospital after a cycle fall with cervical spine hyperflexion and mild occipital head trauma. She was wearing a helmet when the fall occured.

Her Glasgow Coma Scale score at admission was 13 with a normal physical and neurological examination, blood pressure levels and blood investigation including coagulation. She complained only headache and have vomited three times after the trauma.

The patient underwent to cervical X-ray that showed normal alignment and normal craniovertebral junction (CVJ) anatomy without evidence of fractures or atlo-assial disclocation. A CVJ CT depicted a retroclival hyperdense mass lesion with no significant mass effect and without evidence of skull fracture. An MR examination showed the presence of an retroclival epidural soft mass extending from the clivus to the upper tract of cervical spinal cord. The mass was isointense to the spinal cord on T1 weighted images and hyperintense on T2-W images as an epidural haematoma. The patients was treated conservatively with a good outcome. An MR follow-up at 2 and 6 month demonstrated the complete resolution of the REH.

The etiology of REH remains still unclear and it could be due to either clivus fracture or CVJ ligament disruption.

The majority of cases involve pediatric patients after a traumatic event, it's probably that ligamentous laxity at the CVJ in this age group predispose them to ligamentous injury and to the formation of REH.

In spite of this, it's important to consider the occurrence of REH also in young population without an evidence of fracture.

\section{PO8:4}

DIFFUSION TENSOR IMAGING OF THE CORPUS CALLOSUM IN PATIENTS WITH POSTTRAUMATIC DIFFUSE AXONAL INJURY; A 3 TESLA STUDY

B. Goraj ${ }^{1}$, R. Van Koutrik ${ }^{1}$, P. Handels ${ }^{1}$, E. Reuterink ${ }^{1}$,

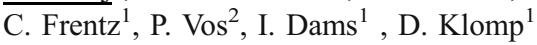

${ }^{1}$ Department of Radiology, University Medical Center

Nijmegen, Nijmegen, The Netherlands; ${ }^{2}$ Department

of Neurology, University Medical Center Nijmegen, Nijmegen, The Netherlands

\section{AIM}

To optimize Diffusion Tensor Imaging (DTI) acquisition technique on 3 Tesla scanner and postprocessing methodology in order to allow adequate discrimination of ROIs within the white matter with the assessment of FA and ADC values. Subsequently, the goal was to compare those parameters and Fiber Tracking (FI) obtained on the brains of the normal volunteers with those of patients after Severe Traumatic Brain Injury (STBI) with diagnosis of Diffuse Axonal Injury (DAI).

METHOD

3 T DTI sequences (Siemens) with different scanning parameters were applied while scanning 5 healthy volunteers to establish which ones provide quality DTI data set crucial for ROIs discrimination; FI and FA / ADC measurements. Corpus Callosum (CC) was chosen as a ROI for comparison between the DTI parameters of normal brains with the brains of patients with clinically diagnosed DAI.

\section{RESULTS}

The spatial scan resolution and the amount of scanning directions proved to be crucial for the quality of imaging but also for a reliable determination of $\mathrm{CC}$, taking into account this structure dimensions; FA values showed variability dependent on voxel size.

The FA and ADC values measured respectively in 3 ROIs within Corpus Callosum in healthy volunteers and in DAI patients showed significant differences.

\section{CONCLUSIONS}

Spatial scan resolution and amount of scanning directions influence the qualitative and quantitative DTI scan properties but have to be balanced with a total scanning time while imaging patients in severe clinical status after STBI. Partial volume effects while choosing voxel size have to be considered in terms of volume of chosen ROIs.

DTI parameters of patients after STBI with suspected DAI showed significant differences when compared with those of healthy volunteers indicating that this method can be useful for DAI detection and quantification.

\section{Infectious Disorders}

\section{PO9:1}

\section{REVERSIBLE RESTRICTED DIFFUSION OF GENU OF CORPUS CALLOSUM}

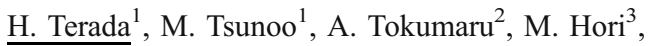
M. Shiraga ${ }^{3}$, Y. Morikawa ${ }^{4}$

${ }^{1}$ Toho University Sakura Medical Center, Sakura, Japan;

${ }^{2}$ Tokyo Metropolitan Geriatric Hospital, Tokyo, Japan;

${ }^{3}$ Toho University Omori Medical Center, Tokyo, Japan;

${ }^{4} \mathrm{Kkr}$ Tachikawa Hospital, Tachikawa, Japan

MR imaging of a 14-year-old man with influenza encephalopathy demonstrated T2 prolongation and restricted diffusion involving the splenium and genu of corpus callosum and symmetrically involving the cerebral hemispheric white matter. The body of corpus callosum was intact. His neurological signs and symptoms were mild, and recovery was complete within a week. Follow-up MR imaging with a 6-day interval revealed complete resolution of these corpus callosum lesions and almost complete resolution of cerebral hemispheric white matter lesions. The clinical and radiological courses were identical to those previously reported with a reversible lesion isolated to the splenium, or to the splenium and peripheral fronto-parietal white matter. One case report showed this reversible lesion may involve entire corpus callosum. Our case suggests that this reversible process may involve the genu of corpus callosun without involvement of body of corpus callosum. 


\section{PO9:2}

\section{CLINICALLY MILD ENCEPHALITIS/ENCEPHALOPATHY WITH A REVERSIBLE SPLENIAL LESION IS ASSOCIATED WITH HYPONATREMIA}

H. Tada ${ }^{1}$, J. Takanashi ${ }^{1}$, A. Barkovich ${ }^{2}$, M. Maeda ${ }^{3}$

${ }^{1}$ Kameda Medical Center, Kamogawa, Japan; ${ }^{2}$ University of California San Francisco, San Francisco, USA; ${ }^{3} \mathrm{Mie}$

University School of Medicine, Tsu, Japan

\section{PURPOSE}

The MR imaging finding of a reversible isolated lesion with transiently reduced diffusion in the central portion of the splenium of the corpus callosum (SCC) has been reported in patients with clinically mild encephalitis/encephalopathy, leading to a new clinical-radiological syndrome, clinically mild encephalitis/encephalopathy with a reversible splenial lesion (MERS). We have encountered several MERS patients with associated hyponatremia. The purpose of this study is to evaluate the $\mathrm{Na}$ level in a series of patients with MERS.

METHODS

We retrospectively reviewed $\mathrm{Na}$ levels of 30 patients with newly diagnosed MERS. As disease controls, we evaluated the Na levels of nine patients with other types of encephalopathy and 17 patients with febrile seizures.

RESULTS

$\mathrm{Na}$ was $131.8 \pm 4.1 \mathrm{mmol} / \mathrm{l}$ (mean $\pm \mathrm{SD}$ ), ranged from 121 to 141 in patients with MERS; $136.6 \pm 2.5 \mathrm{mmol} / \mathrm{l}$ in those with other type of encephalopathy; and $136.2 \pm 2.6 \mathrm{mmol} / \mathrm{l}$ in those with febrile seizures (Fig. 1). Ten of the 30 patients with MERS had Na<130 mmol/l; no patient with other types of encephalopathy or febrile seizures had $\mathrm{Na}$ values this low. Twenty-five of the 30 patients with MERS had $\mathrm{Na}<$ $136 \mathrm{mmol} / \mathrm{l}$. There were significant differences between the Na levels of patients with MERS and those with other types of encephalopathy $(p<0.01)$, and between those of patients with MERS and those with febrile seizures $(\mathrm{p}<0.01)$.

CONCLUSIONS

MERS seems to have cerebral edema due to hyponatremia as a underlying pathophysiology. It is not possible, from the clinical perspective, to completely separate MERS from hyponatremic encephalopathy or to rule out hyponatremia as a contributing factor of MERS.

\section{PO9:3}

\section{CONVENTIONAL AND DIFFUSION-WEIGHTED IMAGING FINDINGS IN A PATIENT WITH AN ISOLATED ABSCESS OF THE CLIVUS}

J. Soares-Fernandes, J. Valle, N. Morais, M. Ribeiro, Z. Magalhães, J. Rocha, J. Moreira-Costa

Hospital S. Marcos, Braga, Portugal

\section{INTRODUCTION}

Involvement of the clivus by infectious processes is a rare finding which may cause significant diagnostic challenge. The main differentials to consider are sphenoid sinus mucopyoceles with posterior erosion to the clivus and neoplasms, especially metastases. Accurate preoperative diagnosis is of the utmost importance because of its implications for surgical approach.

CASE REPORT

A 44-year-old woman presented with a 1-month history of progressively worsening headaches, nausea and vomiting. Neurological examination was unremarkable. Hess screen chart examination revealed incomplete left sixth nerve palsy. CT and thin-section multiplanar reformations showed a midline mass lytic lesion, involving the upper clivus and sellar floor, without communication with the sphenoid sinus. MRI demonstrated a cystic-necrotic lesion, with a hypointense rim on T2-weighted images, thick peripheral enhancement, and central restricted diffusion. Laboratory analysis failed to reveal increased infectious or tumour markers. No primary malignancy was detected on cervical, chest, abdominal and pelvic computed tomography. After 3 weeks of treatment with high-dose clindamycin and gentamicin without clinical or imaging improvement, the patient underwent surgery. An abscedated collection was found. Gram's stain revealed only inflammatory cells. Bacterial, mycobacterial, and fungal cultures were negative, presumably because of administered antibiotics. After surgery, the patient's complaints improved.

\section{DISCUSSION}

In the present case, the lack of contiguity between the abscess and the sphenoid sinus excludes mucopyocele. Infectious processes should be included in the differential diagnosis of a lytic lesion of the clivus, irrespectively of the absence of clinical or laboratory signs of infection. As in brain abscesses, the finding of central restricted diffusion in a cystic, peripheral enhancing mass of the clivus may suggest an infectious etiology.

\section{PO9:4}

\section{HYDATID DISEASE OF THE CNS}

$\underline{\text { V. Katsaros }}^{1}$, P. Lampropoulou ${ }^{1}$, M. Mitropoulou ${ }^{1}$, A. Nikolaou', S. Kavvadias ${ }^{2}$, C. Drossos ${ }^{2}$

${ }^{1}$ IKA Oncology Hospital, Department of CT and MRI, Athens, Greece; ${ }^{2}$ Athens General Hospital, Department of Modern Imaging Modalities, Athens, Greece

Hydatid disease (HD) is a unique parasitic disease that is endemic in several countries of the world produced by the larval stage of Echinococcus. HD can occur almost anywhere in the body and demonstrates a variety of imaging features that vary according to growth stage, associated complications, and affected tissue. Radiologic findings range from purely cystic lesions to a completely solid appearance. Calcification is more common in HD of the liver, spleen, and kidney. CT best demonstrates cyst wall calcification and cyst infection. $\mathrm{CT}$ and MR imaging may 
demonstrate cyst wall defects as well as the passage of contents through a defect. MR imaging is superior in demonstrating neural involvement. Familiarity with atypical manifestations of hydatid disease may be helpful in making a prompt, accurate diagnosis. Three very rare cases with involvement of the central nervous system are demonstrated.

\section{PO9:5}

\section{UNUSUAL CASES OF BRAIN INFLAMMATION/INFECTION}

\section{$\underline{\text { V. Katsaros }}^{1}$, P. Lampropoulou ${ }^{1}$, M. Mitropoulou ${ }^{1}$,} M. Theofanopoulou ${ }^{2}$, Z. Nikolakopoulou ${ }^{3}$, C. Drossos ${ }^{3}$

${ }^{1}$ IKA Oncology Hospital, Department of CT and MRI, Athens, Greece; ${ }^{2}$ Ionia Diagnostic Center, Athens, Greece; ${ }^{3}$ Athens General Hospital, Department of Modern Imaging Modalities, Athens, greece

To present some unusual cases of brain inflammatory processes which we encountered in our daily routine the last 10 years.

10 rare cases of brain inflammation diagnosed mainly by MRI in correlation with clinicolaboratory findings will be demonstrated. They include: Epstein-Barr viral encephalitis, neurosarcoidosis, brain nocardiosis, parenchymal and intraventricular echinococciasis, mucormycosis, toxoplasmosis in non-AIDS patient, leptomeningeal staphylococcosis, brain staphylococcus abscess after cholesteatoma surgery with associated venous sinus thrombosis and PML (papova virus) without any other associated pathology.

\section{PO9:6}

\section{THE UTILITY OF T-2 WEIGHTED IMAGES} FOR EVALUATING THE DIAGNOSIS AND PROGNOSES OF PARTIAL STATUS EPILEPTICUS: CASE REPORT

\author{
M. Vendrame, Z. Hannef, H. Ming, M. Jacobson, \\ S. Azizi \\ Temple University, Philadelphia, USA
}

The causes, prognoses, and appropriate treatments of status epilepticus vary according to electrographical and clinical evaluation. We have observed MRI lesions evident in T-2 weighted sequences in one case of partial status epilepticus. The patient presented with clinical signs and symptoms of viral encephalitis. T2-weighted MRI showed increased signal involving the entire left mesial temporal lobe, insular cortex and inferior frontal regions, which was suggestive of herpes encephalitis. Diffusion weighted sequences were within normal limits. EEG showed left periodic epileptiform discharges. Paraneoplastic limbic encephalitis was ruled out with negative anti-Hu and other tumor markers along with normal CT of chest, abdomen and pelvis. Repeated MR brain at 2 months after the event showed complete resolution of prior observed lesions. This case adds to the existing literature reporting MR abnormalities in patients with partial status epilepticus and suggests that differential diagnosis with infectious and paraneoplastic processes has to be taken into consideration. More cases need to be followed to ultimately evaluate the diagnostic and prognostic dignificance of T-2 weighted lesions in status epilepticus.

\section{PO9:7}

\section{CENTRAL NERVOUS SYSTEM TUBERCULOSIS PRESENTING AS CEREBRAL VENOUS THROMBOSIS}

M. Arantes Da Silva, D. Marques, R. Correia De Abreu, F. Rosa, R. Garcia, C. Alves, J. Resende Pereira Hospital Pedro Hispano, Matosinhos, Portugal

\section{INTRODUCTION}

The cerebral venous thrombosis (CVT) of infectious cause is rare in developed countries and CVT of tuberculosis aetiology is exceptional. In the literature, CVT as initial symptom of tuberculosis was seldom described.

OBJECTIVE

Correlation of imaging and clinical findings of CVT associated with central nervous system tuberculosis

\section{RESULTS}

32 years old woman, previously healthy. Two months before the hospitalization, she had an abortion (at 21 weeks of gestation) of a dead foetus. She was admitted in the urgency service, because of intense headaches with two days of evolution, associated in the day of hospitalization, to a confusional state, dysphasia and psychomotor agitation. Cerebral MRI with venogram demonstrated straight sinus thrombosis and multiple areas of high signal on T2-weighted images, with supratentorial preponderance. Laboratory investigations showed no alterations. She initiated heparin and midazolam. Since the second day of hospitalization, she had fever, rise in serum $\mathrm{C}$ reactive protein, hiponatremy and leukocyturia. Serologies for HIV, hepatitis and syphilis were negative. Thorax radiography revealed discreet infiltration in the right pulmonary base. She initiated amoxicillin and clavulanic acid and at the sixth day of hospitalization she was vigil and oriented, with discreet limitation in the left eye abduction; without motor deficits. At the eighth day she had a sudden dyspnoea and severe desideration, depression of the conscience state, aphasia and left hemiparesis. Echocardiogram was normal. Thoracic CT without signals of thromboembolism. She repeated cerebral MRI and venogram that revealed multiple rounded lesions, with contrast enhancement on T1-weighted images with contrast, and partial recanalization of the straight rectum. Cerebrospinal fluid (CSF) had $70 \mathrm{cel} / \mathrm{uL}$ with a polimorfonuclear preponderance, high protein levels $(5,6 \mathrm{~g} / \mathrm{L})$ and glicosis $(6,39 \mathrm{mg} / \mathrm{dl})$. Research of DNA for complex Mycobacterium tuberculosis in CSF was positive with subsequent isolation of the same agent in culture. She initiated antituberculous therapy and dexamethasone at the nineth day of hospitalization, with a favourable evolution.

CONCLUSION

The cerebral tuberculomas are a rare entity, appearing, as a rule, in immunocompromised patients. They are usually solitary lesions 
$(66 \%)$. Cerebral venous thrombosis is rare, but can be associated to multiple pathologies, namely infectious diseases. However, the bibliographic revision shows rare cases associated to central nervous system tuberculosis.

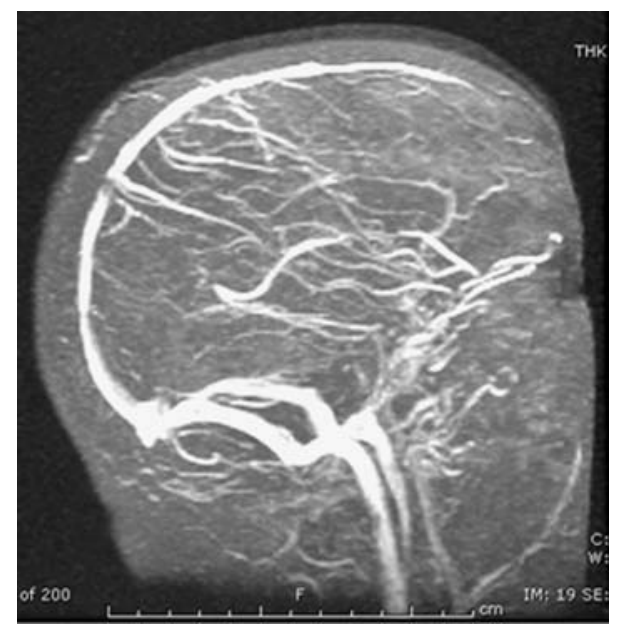

\section{Pediatrics}

\section{PO10:1}

\section{ASSOCIATED ABNORMALITIES IN FETUSES WITH VENTRICULOMEGALY MRI INVESTIGATION}

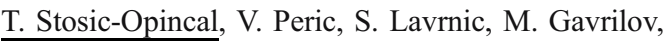

A. Ljubic

MR Center, Clinical Center of Serbia, Belgrade, Serbia Montenegro

\section{PURPOSE}

To determine frequency and type of cases in which fetal magnetic resonance imaging (MRI) provides additional clinically relevant information in fetuses with sonographically diagnosed ventriculomegaly.

\section{METHODS}

Nineteen cases presenting with different degrees of ventriculomegaly on ultrasound imaging were investigated by MRI. The mean gestational age of fetuses was 31.3 weeks.

\section{RESULTS}

Fetal MRI confirmed the sonographic diagnosis in 15 of 19 cases, revealed additional findings in 12 of 15 cases. Three patients with the diagnosis of isolated ventriculomegaly were found on MRI to have ventricles that measured within the range of normal (less or equal to $10 \mathrm{~mm}$ atrial diameter).

In the marked ventriculomegaly group (10 cases), 2 cases of complete agenesis of the corpus callosum, 2 cases of intraventricular hemorrhage, 3 cases of polymicrogyria, 2 cases of porencephaly and 1 case of craniosynostosis were detected. In the group of borderline ventriculomegaly (5 cases), 2 cases of partial agenesis of the corpus callosum, 2 cases of porencephaly and one case of lizencephaly were detected.

CONCLUSION

MRI is useful additional investigation in fetuses with ventriculomegaly, especially in visualizing associated abnormalities, such as intraventricular hemorrhage, porencephaly, callosal dysgenesis, and cortical migrational abnormalities.

\section{P010:2}

\section{MR IMAGING OF NEONATAL PYRUVATE DEHYDROGENASE DEFICIENCY DUE TO PDHA1 GENE MUTATION}

J. Soares-Fernandes ${ }^{1}$, R. Teixeira-Gomes ${ }^{2}$, R. Cruz ${ }^{3}$, M. Ribeiro $^{1}$, Z. Magalhães ${ }^{1}$, J. Rocha ${ }^{1}$

${ }^{1}$ Hospital de S. Marcos, Braga, Portugal; ${ }^{2}$ Hospital Pedro Hispano, Matosinhos, Portugal; ${ }^{3}$ Hospital Geral de Sto. António, Porto, Portugal

\section{INTRODUCTION}

Pyruvate dehydrogenase (PDH) deficiency results from mutations in the mitochondrial PDH complex, mostly in the E1- ${ }^{\alpha}$ subunit (PDHA1 gene). The diagnosis of PDH deficiency in neonates is difficult because the clinical features are non-specific and biochemical confirmation may take a long time to complete. Therefore, a better known of the imaging appearance would be useful, in order to suggesting this specific diagnosis.

\section{CASE REPORT}

A 38-week gestation male neonate was moved to intensive care after birth because of respiratory distress. Initial neurological examination revealed an absent swallowing reflex, an exaggerated Moro reflex, and axial hypotonia. MRI at 7 days of life showed colpocephaly, a dysgenetic corpus callosum, prominent bilateral subependymal cysts in the caudothalamic groove and upper lateral ventricles, and generalized hypointensity of the white matter on T1-weighted images with cystic change in the anterior temporal lobes. Myelination was normal. No cortical malformations were detected. MR spectroscopy (TE, $135 \mathrm{~ms}$ ) demonstrated a lactate doublet at $1.33 \mathrm{ppm}$. Elevated plasma and CSF lactate and pyruvate levels, with normal lactate to pyruvate ratio were present. Fibroblast biochemical analysis confirmed significantly decreased PDH activity. Mutation analysis detected a mosaicism for the $\mathrm{R} 302 \mathrm{H}$ mutation in the PDHA1 gene.

\section{DISCUSSION}

Colpocephaly and white matter cysts are non-specific imaging features of neonatal PDH deficiency. Callosal malformations are more characteristic since few metabolic disorders cause brain malformations. The finding of bilateral subependymal cysts was previously reported in two neonates with PDH deficiency caused by different mutations. All patients were term neonates, the cysts were present at birth, and had a similar distribution (not only in the caudothalamic grooves but also in the upper lateral ventricles). Thus, they unlikely represent evolution of germinal matrix hemorrhages, and may play a role in suggesting this specific diagnosis. Further neonatal imaging reports are needed. 


\section{P010:3}

\section{A CASE SERIES OF FETAL MRI}

\author{
$\underline{\text { B. Schmitz }}^{1}$, D. Boll ${ }^{1}$, S. Klein ${ }^{1}$, A. Aschoff ${ }^{1}$, A. Ernst ${ }^{1}$, \\ R. Terinde ${ }^{2}$ \\ ${ }^{1}$ Clinic for Diagnostic and Interventional Radiology, \\ University Hospitals Ulm, Ulm, Germany; ${ }^{2}$ Dept. \\ of Gynecology, University Hospitals Ulm, Ulm, Germany
}

\section{PURPOSE}

Fetal MRI of the brain is becoming a valid extension to ultrasound in prenatal diagnostics. Ongoing improvements in scanner hardware and sequence technique have made it possible to examine the fetal brain without sedation or relaxation. We present a case series with typical pathologies of the fetal brain.

\section{MATERIALS AND METHODS}

12 patients (6 between 20-23 weeks, 6 between 31-37 weeks of gestation)were referred for MRI from gynecologists if prenatal ultrasound was not conclusive. Patients were without sedation. After triplanar scout views a coronal HASTE stack was acquired. The subsequent series was planned to go through both fetal eyes disregarding other orientations. On the thereby generated eye-parallel images the following axial, coronal and sagittal HASTE sequences were planned. Fetal position was checked after each sequence and sequence orientation for the next sequence always adjusted to the last measured sequence to keep track with fetal movements. Field of view and number of slices were adjusted to the size of the fetal brain. Typical parameter: Siemens 1,5 T Avanto system with phased array coils, slice thickness $3 \mathrm{~mm}, 256 \times 256$ matrix, TR $1000 \mathrm{~ms}$, TE $69 \mathrm{~ms}$, bandwidth $490 \mathrm{~Hz} / \mathrm{Px}$. Additional contrasts (T1, TrueFISP, DWI) were acquired if needed in special cases.

\section{RESULTS}

Image quality was always sufficient for diagnosis. Imaging time ranged between $15 \mathrm{~min}$ for triplanar HASTE images only and $50 \mathrm{~min}$ for multiple contrasts and in cases of severe fetal movements rendering repeated measurements necessary. We observed corpus callosum agenesia in 2 and hypoplasia in 5 cases, hydranencephalus in one case, MCA infarcts in one case, severe hydrocephalus in one case and occipital encephalocele in one case (figure). Other cases were mixed pathologies.

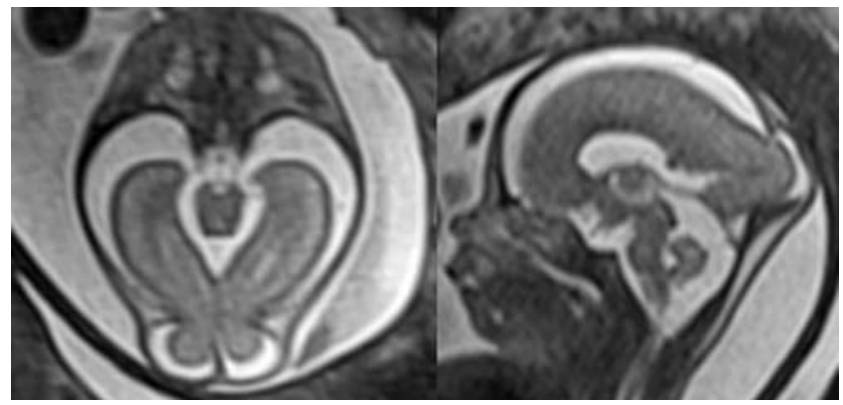

\section{CONCLUSION}

Fetal MRI is a fast and reliable examination able to discern relevant fetal brain pathologies with high diagnostic confidence.

\section{PO10:4}

\section{DYNAMIC 3D MRA: INTEREST IN INTRACRANIAL} VASCULAR DISEASES EVALUATION IN CHILDREN

\section{E. Schmitt, C. Moret, R. Anxionnat, S. Foscolo, S. Bracard} Service De Neuroradiologie Diagnostique Et Therapeutique, Nancy, France

\section{PURPOSE}

Dynamic 3D MRA is based on sequential acquisition of images after contrast bolus injection. The technique we used (TRICKS, GE Healthcare) was recently developed and provides vascular dynamic information which seems to be well appropriate for cerebro-vascular diseases study in adult. To our knowledge, this technique is poorly described in children. The purpose of this study is to adapt the technical parameters for children and to evaluate the interest of ECTRICKS for cerebrovascular disease in children.

METHOD

We used it in different conditions in 10 children (3 days to 13 years) on a $1.5 \mathrm{~T}$ GE MRI with good results.

\section{RESULT AND CONCLUSION}

Dynamic MRA is technically feasible with a good result in children, even very young. In this short series it gave us valuable information in various cerebrovascular diseases in children such as superficial vascular malformation, cerebral veinous thrombosis, sinus pericranii, ..... To our opinion, this technique was helpful in many situations in children and could become the first choice MRA sequence for the diagnosis and evaluation of vascular diseases but the evaluation needs to be pursued.

\section{P010:5}

\section{SANDHOFF DISEASE: A CASE REPORT}

M. Sallei ${ }^{1}$, L. D’Alessandro ${ }^{1}$, L. Regnicolo ${ }^{2}$, G. Polonara ${ }^{2}$, O. Gabrielli ${ }^{3}$, S. Salvolini ${ }^{4}$, U. Salvolini ${ }^{2}$

${ }^{1}$ Radiologia Università Politecnica delle Marche, Ancona, Italy; ${ }^{2}$ S.O.D. Clinica di Neuroradiologia Azienda Ospedaliero-Universitaria Ospedali Riuniti, Ancona, Italy; ${ }^{3}$ S.O.D. di Pediatria Azienda Ospedaliero-Universitaria Ospedali Riuniti, Ancona, Italy; ${ }^{4}$ Clinica di Oftalmologia Azienda Ospedaliero-Universitaria Ospedali Riuniti, Ancona, Italy

\section{PURPOSE}

Sandhoff's disease (SD) or GM2 gangliosidosis type 0 is a rare autosomal recessive disorder caused by a deficiency of both $\mathrm{A}$ and B $\beta$-hexosaminidase with accumulation of GM2 and GA2 gangliosides, especially in neurons and viscera.

Infantile SD is characterized by early onset of severe and progressive symptoms (usually in the first year of life) that include psychomotor retardation/regression, macrocephaly, seizures, blidness $(90 \%$ with cherry-red spots in the macula), deafness, hepatosplenomegaly and death by 4 years of age. 
MRI demonstrates thalamus signal hypointensity on T2-weighed and hyperintensity on T1-weighed images, white matter signal hyperintensity on T2 W images, cortical atrophy, decreased NAA and increased Cho peaks. We describe a patient with infantile SD. METHODS

The patient was a Chinese girl of consanguineous parents with seizures since her first year of life and psychomotor retardation. Clinical examination at 15 months demonstrated muscle atrophy, generalized hypotonia except for upper limb hypertonia with brish deep tendon reflex; no hepatosplenomegaly or retinal alterations. RESULTS

Serum assays showed A and B $\beta$-hexosaminidase deficiency.

White matter, corpora striata and caudate heads showed high signal on T2-weighed images; the thalamus was hyperintense on T2-weighted and hypointense on T1-weighted images; the NAA peak was decreased and the Cho peak was increased.

\section{CONCLUSIONS}

Serum assays showing A and B $\beta$-hexosaminidase deficiency led to the diagnosis of SD. However, MRI and MRS demonstrated the typical alteration of the disease. This is important for early diagnosis, especially in the case of heterozygous consanguineous parents at high risk of having other children with SD.

\section{PO10:6}

\section{SEPTO-OPTIC DYSPLASIA WITH OLFACTORY TRACT HYPOPLASIA}

M. Ribeiro, J. Fernandes, A. Machado, J. Cerqueira,

J. Rocha

Hospital Sao Marcos, Braga, Portugal

\section{INTRODUCTION}

Septo-optic dysplasia (Sod) is a rare developmental anomaly, characterized by optic nerve hypoplasia and septum pellucidum (SP) agenesis, and is frequently associated with hormonal deficiencies.

\section{CASE REPORT}

We present the case of a 4-month-old male patient with a pre-natal diagnosis of a SP cyst. The child had an otherwise unremarkable birth history and physical development, having reached appropriately all childhood milestones.

Transfontanelar ultrasonographic examination revealed SP absence and mild enlargement of lateral ventricles.

Magnetic Resonance imaging confirmed the complete SP absence and revealed right optic nerve atrophy. Olfactory tract and sulci hypoplasia were also noted. There was diffuse white matter atrophy, with thinning of corpus callosum which otherwise was well formed, and large ventricles with squared-off appearance of the frontal horns. The pituitary gland was small but well formed. There was no evidence of schizencephaly.

Ophthalmologic examination confirmed right optic disc hypoplasia. Endocrine studies showed no abnormalities. Genetic testing (Hesx1 gene) was not performed.

CONCLUSION

The diagnosis of Sod is established when hipoplasia of the optic disc is observed in association with agenesis of the septum pellucidum. This case represents a very rare association of Sod and olfactory tract hypoplasia.

The etiology of Sod remains uncertain, with genetic abnormalities (in continuum with lobar holoprosencephaly) and intrauterine insults having nevertheless been proposed as etiological factors.

In several cases, the mutation in the homeobox gene Hesx 1 was shown to be implicated with mild pituitary hypoplasia or Sod. Transgenic mice lacking the homologue gene of human Hesx 1 exhibit a phenotype including SP abnormalities, hypoplastic optic vesicles, pituitary dysplasia and defective olfactory development. In humans, Sod also presents a highly variable phenotype. The authors believe that bulb and tract olfactory hypoplasia should be included in the variable phenotype of Sod and could be explained by Hesx 1 mutation.

\section{P010:7}

\section{HEMIMEGALENCEPHALY AND NEURONAL MIGRATION DISORDERS ASSOCIATED \\ TO REFRACTORY EPILEPSY: MAGNETIC RESONANCE IMAGING FINDINGS IN 14 CHILDREN}

\author{
V. Peric $^{1}$, T. Stosic-Opincal ${ }^{1}$, S. Lavrnic ${ }^{1}$, N. Jovic ${ }^{2}$, \\ V. Brankovic ${ }^{2}$ \\ ${ }^{1}$ MR Center, Clinical Center of Serbia, Belgrade, Serbia - \\ Montenegro; ${ }^{2}$ Clinic for Child Neurology and Psychiatry, \\ Clinical Center of Serbia, Belgrade, Serbia - Montenegro
}

\section{OBJECTIVES}

Patients with neuronal migration disorders and hemimegalencephaly can present with early onset epilepsy which is often refractory to medical treatment and associated to impaired neurological development. Our aim is to present the MRI findings in children with medically intractable epilepsy and pathologically confirmed neuronal migration disorders, including unilateral cortical dysplasia and hemimegalencephaly.

\section{PATIENTS AND METHODS}

In 14 children ( 8 boys and 6 girls), aged 2 to 8 , with medically intractable epilepsy brain MRI was performed on 1.5 Tesla scaner (Siemens Avanto).

\section{RESULTS}

Common MRI findings included abnormal gyral formation, abnormal cortical thickness, hemimegalencephaly, blurring of the gray matter-white matter junction and abnormal signal in gray matter and subcortical white matter on T2-weighted images.

\section{CONCLUSION}

These results demonstrate that magnetic resonance imaging reflects various structural changes closely related to epileptogenesis in unilateral cortical dysplasia and hemimegalencephaly. Detected abnormal signals correspond to various degrees of dyslamination and morphologic abnormalities of neurons and glial cells in the gray matter, and to dysmyelination, ectopic clustering of dysplastic neurons, glial proliferation, and necrotic changes in the white matter. 


\section{P010:8}

MRI FINDINGS AT THE MITOCHONDRIAL DNA A8344G TRNALYS MUTATION ASSOCIATED WITH RECURRENT MIGRATORY BRAIN STEM STROKE-LIKE EPISODES

E. Papadaki, I. Zaganas, H. Latsoudis, P. Vorgia, M. Spilioti, A. Plaitakis University Hospital of Heraklion, Heraklion, Greece

\section{BODY}

The A8344G tRNALys mitochondrial DNA mutation is typically associated with MERRF syndrome. We studied a 17-year-old female who had a long-standing MERRF-like disorder characterized by deafness, seizures, peripheral neuropathy, ataxia, endocrinopathy and elevated blood and CSF lactate and who recently experienced two distinct episodes of clinical worsening associated with brain stem stroke-like lesions. The first occurred in August 2006 and it was clinically manifested by oculomotor disturbances and severe gait ataxia with partial recovery occurring over the ensuing months. Brain MRI revealed a hyperintense focal lesion in the tegmentum of the right midbrain. In January of 2007, the patient experienced the abrupt onset of swallowing difficulties, dysarthria and increasing gait ataxia. Repeated brain MRI revealed a new hyperintense lesion in the right medulla while the previous midbrain lesion showed substantial resolution. Both lesions showed high signals on DWI with normal apparent diffusion co-efficient values, differentiating them from acute ischemic lesions. Magnetic Resonanse single-voxel Spectroscopy applied at the basal ganglia was normal. DNA analysis revealed the A8344G tRNALys mitochondrial mutation in almost homoplasmic state. The A3243G mutation that is typically associated with MELAS and occasionally with the MERRF/MELAS overlap syndrome was absent. Hence, the A8344G tRNALys mutation can produce recurrent migratory stroke-like episodes involving the brain-stem.

\section{P010:9}

\section{BRAIN TUMORS IN THE FIRST 2 YEARS OF LIFE:} NEUROIMAGING AND HISTOLOGIC FINDINGS

\author{
I. Nikas $^{1}$, M. Theofanopoulou ${ }^{1}$, A. Stefanaki ${ }^{2}$, A. Michail ${ }^{3}$, M. Zarifi ${ }^{1}$, \\ G. Pitsoulakis ${ }^{1}$, C. Hadjigeorgi ${ }^{1}$ \\ ${ }^{1}$ Imaging Dpt, Aghia Sofia Children's Hospital, Athens, \\ Greece; ${ }^{2}$ Pathology Dpt, Aghia Sofia Children's Hospital, \\ Athens, Greece; ${ }^{3}$ Pathology Dpt, Aglaia Kyriakou, \\ Children's Hospital, Athens, Greece
}

\section{PURPOSE}

Brain tumors occuring in the first 2 years of life constitute a special group as their clinical, imaging, histological and therapeutic characteristics differ from those of the entire pediatric population. Purpose of this study is to describe and correlate the neuroimaging and histopathological findings of brain tumors presenting in this period.

\section{METHODS}

During the 7-year period (1999-2006) 47 intracranial tumors were diagnosed in children in their first 2 years of life (22 males and 25 females) in the two pediatric hospitals of Athens.

\section{RESULTS}

Supratentorial tumors with a higher percentage (57.4\%) than infratentorial ones $(42.6 \%)$ in our series, consisted of 6 supra/ parasellar astrocytomas, 5 ependymomas, 3 desmoplastic infantile gangliogliomas, 3 choroid plexus papillomas, 2 gangliogliomas, 2 Primitive Neuroectodermal Tumors (PNET) and 1 case each of teratoma, Dysembryoblastic Neuroepithelial Tumor (DNET), craniopharyngioma, subependymal giant cell astrocytoma, choroid plexus carcinoma and medulloepithelioma. In the posterior fossa 7 astrocytomas, 6 medulloblastomas, 5 ependymomas and 2 atypical teratoid rhabdoid tumors were found.

\section{CONCLUSIONS}

Neuroimaging was valuable in the pre-therapeutic evaluation allowing not only a detailed description of the tumor but also guiding therapeutic decisions. Histopathology established the final diagnosis based on the neoplasms morphological and immunophenotypic features.

\section{P010:10}

\section{MAGNETIC RESONANCE IMAGING OF BILATERAL MESIAL TEMPORAL SCLEROSIS IN CHRONIC KERNICTERUS}

M. Mendes, F. Costa, A. Silva, C. Pontes, M. Rosas

Hospital De S.João, Oporto, Portugal

\section{INTRODUCTION}

Kernicterus is an encephalopathy due to neuronal toxicity of unconjugated bilirrubin in the first days of life. The most characteristic pattern of neuropathologic lesions is symmetric and highly selective involvement of the basal ganglia.

We describe a case of kernicterus where MRI imaging demonstrated bilateral high signal and volume loss in the hippocampus. CASE REPORT

A 9-year-old black girl was admitted for investigation of dystonia and severe developmental delay. The patient had no history of perinatal asphyxia, metabolic abnormalities, or $\mathrm{ABO} / \mathrm{Rh}$ isoimmunization. At 3 days of age, she presented with jaundice, fever and irritability. After treatment with antibiotics and phototherapy, axial hypotonia and limitation of vertical gaze movements were noted. At 6 months old, serious psicomotor delay and generalized dystonia developed.

Currently, neurological examination revealed mental retardation, dystonia and spastic tetraparesis. Brain MRI demonstrated bilaterally symmetric hiperintensities in the globus pallidus and subthalamic nucleus. Bilateral hippocampal volume loss and high signal, resembling bilateral mesial temporal sclerosis, was also observed. Blood analyses were normal, including copper and ceruloplasmin. The screening for mitochondrial disorders, includ- 
ing muscle biopsy, respiratory chain enzymes activity and analysis for mutations of mitochondrial DNA, was negative.

The patient was treated with trihexyphenidyl until $30 \mathrm{mg} /$ day, with improvement of the generalized dystonia.

DISCUSSION

MRI findings in chronic kernicterus are characteristic, with bilateral high signal of the pallida and subthalamic nucleus, but non-specific, and can be found in various disorders, such as hypoxic-ischemic encephalopathy, mitochondrial disorders, inborn errors of metabolism, hemolytic-uremic syndrome and carbon monoxide poisoning.

Although mesial temporal sclerosis is a common pathological finding in patients with kernicterus, it is only exceptionally described in MRI reports. Therefore we believe that hippocampal sclerosis, demonstrated by MRI, plays a role in adding specificity to the imaging diagnosis of kernicterus.

\section{PO10:11}

\section{THE MRI AND 1 HMRS IN AUTISTIC CHILDREN}

B. Kubas $^{1}$, J. Walecki ${ }^{2}$, E. Tarasow ${ }^{1}$, W. Kulak ${ }^{1}$, K. Kaminska ${ }^{2}$, W. Sobaniec ${ }^{1}$

${ }^{1}$ Medical University of Bialystok, Bialystok, Poland;

${ }^{2}$ Polish Academy of Sciences Medical Research Center, Warsaw, Poland

Autism is classified by the WHO as a developmental disability that results from a disorder of the human central nervous system. It is characterized by impaired social interaction, problems with verbal and nonverbal communication, and unusual, repetitive, or severely limited activities and interests.

Although brain imaging studies have reported neurobiological abnormalities in autism, the nature and distribution of the underlying neurochemical irregularities are still unknown. There were some studies suggest that people with autism have abnormal levels of neurotransmitters and other metabolites in the brain.

PURPOSE

The purpose of this study is to assess the role of 1HMRS in the detection of changes in cerebral metabolite levels in autistic children and to compare metabolite alterations to clinical status assessed by Children's Autistic Rating Scale.

MATERIAL

Study group consisted of 14 children, aged 10-15, who were under care of Pediatric Neurology Department of Medical University and Pediatric Department of Central Clinical Hospital MIA. All patients matched the clinical criteria of the disease according to ICD- 10 .

METHOD

The MRI was performed using $1.5 \mathrm{~T}$ scanner, TSE (with T2 inversion) and FLAIR in frontal and transverse planes. 1HMRS (TE-35, TR-1500) with voxel $(2 \times 2 \times 2 \mathrm{~cm})$ localized in centrum semiovale, temporal lobe and cerebellar hemisphere. Spectroscopic data were analysed using the Via $2.0 \mathrm{C}$ software package. We measured ratios of $\mathrm{NAA} / \mathrm{Cr}, \mathrm{Cho} / \mathrm{Cr}, \mathrm{mI}, \mathrm{Glx} / \mathrm{Cr}$ after manual segmentation and compared with Children's Autistic Rating Scale.

\section{RESULTS}

In metabolite concentrations we observed significant differences in $\mathrm{NAA} / \mathrm{Cr}$ in temporal lobe between the control and autistic groups. There was slight increase of $\mathrm{Cho} / \mathrm{Cr}$ ratio in cerebellar hemisphere in autistic group. There was no significant difference in other metabolites. NAA/Cr ratios correlated with scores on Children's Autistic Rating Scale.

\section{CONCLUSIONS}

Metabolite alterations in autistic children suggest that HMRS may be useful tool for characterizing regional neurochemical abnormalities in this disorder.

\section{P010:12}

\section{RADIOLOGIC AND AUDIOLOGIC ASSESSMENT OF CORNELIA DE LANGE SYNDROME}

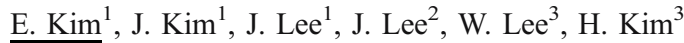 \\ ${ }^{1}$ Deaprtment of Radiology and Research Institute of \\ Radiological Sciences, Yonsei University College of \\ Medicine, Seoul, South Korea; ${ }^{2}$ Department of Clinical \\ Genetics, Yonsei University College of Medicine, Seoul, \\ South Korea; ${ }^{3}$ Department of Otorhinolaryngology, Yonsei \\ University College of Medicine, Seoul, South Korea
}

\section{PURPOSE}

To describe the findings of temporal bone CT in patients with Cornelia de Lange syndrome (CdL) and to compare classical form with mild form in terms of severity of radiologic and audiologic abnormalities.

\section{SUBJECTS AND METHODS}

Ten children ( 6 girls and 4 boys; mean age, 42 months) who were clinically diagnosed as CdL (classical $[n=5]$ and mild form $[n=5]$ ) were enrolled. Temporal bone CT was prospectively performed in all patients. Thirty-two aspects of each temporal bone CT were analyzed: 21 by direct measurement and 11 by visual inspection. Ten age-matched patients with normal temporal bone CT were served as a control group. Audiologic tests were performed in all patients.

\section{RESULTS}

Middle or inner ear abnormalities were identified on CT in 6 of 10 patients, of which classical and mild forms of CdL were present in 5 and 1 patients, respectively. The temporal bone $\mathrm{CT}$ abnormalities in patients with CdL were dysmorphism of malleus and incus $(65 \%)$, hypoplastic cochlea with modiolar deficiency (55\%), enlarged vestibule with focal protrusion $(55 \%)$, soft tissue opacification of tympanomastoid cavity (50\%), and facial nerve dehiscence (15\%), all of which were more prevalent in patients with classical form than those with mild form $(\mathrm{p}<.05)$. The patients with classical form of CdL $(n=5)$ showed more profound hearing impairment. The other 4 patients without CT abnormalities had mild form without hearing impairment except for 1 patient.

\section{CONCLUSION}

All patients with classical form of CdL showed more middle or inner ear structural abnormalities on CT and more severe hearing impairment than those with mild form. CT evaluation of the temporal bone may be helpful for pre-treatment assessment of CdL. 

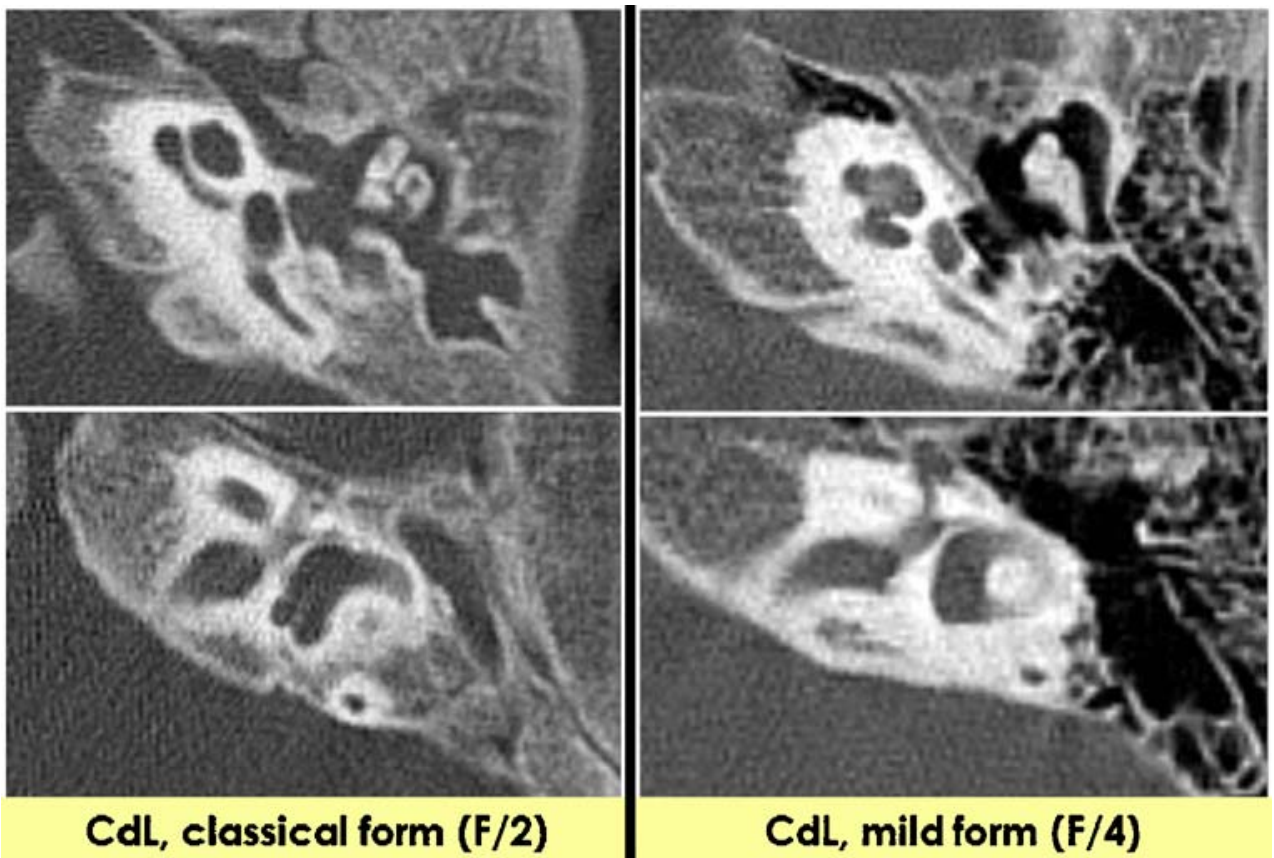

\section{PO10:13}

\section{RAPIDLY DEVELOPING BRAIN ATROPHY \\ IN HEMOLYTIC UREMIC SYNDROME}

F. Kendiroglu ${ }^{1}$, K. Karaali ${ }^{1}$, N. Olgac Dundar ${ }^{2}$, Y. Durum ${ }^{1}$, E. Luleci $^{1}$

${ }^{1}$ Akdeniz University, Department of Radiology, Antalya,

Turkey; ${ }^{2}$ Suleyman Demirel University, Department of

Pediatric Neurology, Isparta, Turkey

\section{PURPOSE}

We present a rare case of a six-month-old boy with diarrheaassociated hemolytic uremic syndrome who rapidly developed cerebral atrophy within eight days.

METHODS

A six-month-old boy was diagnosed as hemolytic uremic syndrome after onset of thrombocytopenia, hyponatremia, acute renal failure and intractable seizures one week following diarrhea. He was referred to our radiology unit for CT and MRI examination. RESULTS

CT findings were bilateral patchy low density areas on occipital lobes and periventricular areas. MRI findings were cerebral edema, intensity changes and restricted diffusion within bilateral basal ganglia, frontal lobes, occipital lobes and periventricular and deep white matter compatible with infarction. After one week of follow up, control MRI examination showed ongoing restricted diffusion within the bilateral basal ganglia and the white matter. Cerebral edema was resolved. The most striking finding was the volume loss of cerebral parenchyma.

CONCLUSION

Basal ganglia involvement is a typical finding in patients with neurological complications of hemolytic uremic syndrome. The unusual finding of this case is the rapid development of cerebral atrophy.

\section{PO10:14}

\section{STURGE-WEBER SYNDROME ASSOCIATED WITH ARACHNOID CYST AND EXTRAAXIAL FAT TISSUE}

$\underline{\text { F. Kendiroglu }}^{1}$, U. Senol ${ }^{1}$, A. Aralasmak ${ }^{1}$, S. Haspolat ${ }^{2}$ ${ }^{1}$ Akdeniz University, Department of Radiology, Antalya, Turkey; ${ }^{2}$ Akdeniz University, Department of Pediatric Neurology, Antalya, Turkey

\section{PURPOSE}

This report presents a unique case of Sturge-Weber Syndrome with right sided temporal arachnoid cyst indenting right orbital wall and the presence of right occipitoparietal extraaxial fat tissue METHODS

A sixteen-year-old-boy with a diagnosis of Sturge-Weber syndrome presented with intractable seizures and progressive deterioration. After the clinical evaluation, the patient was referred to our radiology unit for CT and MRI examination.

\section{RESULTS}

CT images showed right sided occipitoparietal gyryform calcification. MRI showed right sided leptomeningeal angiomatosis, anterior temporal arachnoid cyst indenting right orbital wall and occipitoparietal extraaxial fat tissue.

\section{CONCLUSION}

Sturge-Weber syndrome is characterized by facial vascular nevus associated with ipsilateral leptomeningeal angiomatosis. The 
unusual findings in our case were the right sided temporal arachnoid cyst indenting right orbital wall and the presence of right occipitoparietal extraaxial fat tissue. In our knowledge, this association has not been reported previously in the literature.

\section{P010:15}

\section{COMPARISON OF CLINICAL AND MAGNETIC RESONANCE IMAGING FINDINGS IN CHILDREN WITH HYPOXIC-ISCHEMIC ENCEPHALOPATHY}

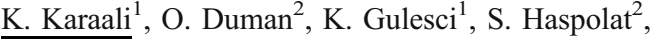 \\ U. Senol $^{1}$ \\ ${ }^{1}$ Akdeniz University, Department of Radiology, Antalya, \\ Turkey; ${ }^{2}$ Akdeniz University, Department of Pediatric \\ Neurology, Antalya, Turkey
}

\section{PURPOSE}

In this study, we retrospectively evaluated the clinical and magnetic resonance imaging findings in children with hypoxicischemic encephalopathy, and compared these findings to evaluate the correlation.

METHODS

Fifty children with clinical diagnosis of hypoxic-ischemic encephalopathy were included in the study. Clinical findings were evaluated by a pediatric neurologist. Gestational age, one minute APGAR score, head circumference, clinical Levene stage and the presence of seizures were recorded. All these findings were compared with MRI findings. For the MRI evaluation, basal ganglia, cerebral cortex, corpus callosum, white matter and ventricular system lesions were noted. Chi-square test and correlation analysis were used for the statistical evaluation.

RESULTS

Presence of seizures and diffuse thinning of corpus callosum were significantly correlated. As well, basal ganglia and cerebral cortical lesions were significantly more frequent in term neonates and microcephalic patients. There was no statistically significant correlation among the other clinical and MRI findings.

\section{CONCLUSION}

Although MR is a powerful tool in the assessment of brain damage resulting from perinatal asphyxia, the correlation of the clinical and imaging findings may be poor.

\section{PO10:16}

\section{PAEDIATRIC STROKE IN EMERGENCY: AETIOLOGY AND NEURORADIOLOGY}

\author{
A. Paonessa ${ }^{1}$, A. Splendiani ${ }^{2}$, N. Limbucci ${ }^{2}$, E. Tozzi ${ }^{3}$, \\ F. Fasano ${ }^{2}$, G. Sirabella ${ }^{1}$, M. Gallucci ${ }^{2}$ \\ ${ }^{1}$ Dept of Neuroradiology, Loreto Nuovo Hospital, Naples, \\ Italy; ${ }^{2}$ Dept of Neuroradiology, University Hospital, \\ L'aquila, Italy; ${ }^{3}$ Dept of Paediatrics, University Hospital, \\ L'aquila, Italy
}

\section{INTRODUCTION AND AIMS}

To critically evaluate aetiology, risk factors and mechanisms of paediatric stroke in emergency.

MATERIALS AND METHOD

39 consecutive paediatric patients with an acute stroke (age ranging between 5 and 16 yrs, average 12) observed in emergency during the last $10 \mathrm{yrs}$, had been retrospectively evaluated. 24 cases underwent MRI in emergency while 4 cases were studied only by CT and 11 had both CT and MRI studies. In 7 cases a catheter angiography was performed within 2 days from admission. Neuroradiological findings, incidence and prevalence of ischemic and hemorrhagic forms and aetiology had been reviewed.

RESULTS AND CONCLUSION

Incidence of paediatric stroke is differently estimated in literature ranging from 2.5 to $13 / 100.000 /$ year, hemorrhagic forms vary from $75 \%$ in studies performed in pre-CT era, to $38-41 \%$ in more recent papers. However, most of the literature concerns cases observed in stable phase, while our experience wants to focus the attention on emergency studies. Among our cases, we found 16 haemorrhagic (41.02\%) and 23 ischemic strokes. Among haemorrhagic forms, 5 cases were due to AVM, 7 to cavernous haemangiomas, 1 to aneurysm and 3 remained cryptogenic. Among ischemic forms, 4 were cardio-embolic, 2 due to sickle cell disease, 2 to hyperhomocysteinemia, 1 to moya-moya disease, 3 to Anti phospholipid syndrome, 1 to Fabry disease, 1 concomitant with $\mathrm{CO}$ intoxication, 4 were due to sinus thromboses, 3 of which related to inflammatory skin or mastoid diseases. 5 cases of cerebral ischemias remained classified as cryptogenic. Our experience confirms the general tendence already reported in literature of a moderate prevalence of ischemic over hemorrhagic strokes, even in acute observation. 8 cases (20,5\%) remain attributed to unidentified causes.

\section{P010:17}

\section{CLINICAL PRESENTATION, CLINICAL COURSE AND NEUROIMAGING FINDINGS IN PEDIATRIC NON-ACCIDENTAL TRAUMA}

B. Foerster, M. Petrou, P. Strouse, P. Sundgren

University of Michigan Ann Arbor USA

\section{PURPOSE}

To describe the clinical presentation and clinical course as well as illustrate the CT and MRI neuroimaging findings in suspected pediatric non-accidental trauma.

\section{METHODS}

We reviewed the medical records and neuroimaging reports of 45 pediatric patients presenting with the clinical suspicion of nonaccidental brain trauma. The clinical presentation, clinical course, and CT/MRI findings were documented in each case. Corroborating information including skeletal fractures and retinal hemorrhages were also noted.

RESULTS

Subdural hematoma was the most common neuroimaging finding seen in 35 out of the 45 cases followed by subarachnoid hemorrhage. 
Findings of ischemia/anoxia correlated with poor clinical outcome. Mental status changes, respiratory depression and seizures were the most common initial presenting symptoms. Two of the patients who presented with seizures had negative HCT and MRI exams decreasing the certainty of the diagnosis of non-accidental trauma.

\section{CONCLUSIONS}

Pediatric non-accidental trauma is not uncommon and is usually initially evaluated with CT. MRI is helpful in dating the injuries and is also more sensitive for the detection of axonal shearing injury and ischemia. Radiologists must maintain a high level of suspicion as the initial provided history can be vague with a highly variable clinical presentation and the physical exam does not always indicate the possibility or severity of intracranial injury. Examples will be illustrated.

\section{PO10:18}

\section{MRI FINDINGS OF DYKE-DAVIDOFF-MASSON SYNDROME REGARDING ONE CASE}

I. Elia, F. Mata, J. Quiles, E. Alonso, E. Yudek, R. Garcia, V. Martinez Hospital General - Unidad De Resonancia Magnetica,

Valencia, Spain

\section{BACKGROUND}

The purpose of our study is to emphasize the irm findings of Dyke-Davidoff-Masson syndrome, so called cerebral hemiatrophy. METHODS

Regarding one case of cerebral hemiatrophy in our hospital, a female 14 years old, who presented tonic-clonic seizures from 7 , with residual hemiparesis on the right side of her body, including her face, after meningitis we made a mri analisys and emphasize the radiological findings.

\section{RESULTS}

MRI findings were cerebral hemiatrophy, unilateral calvarial thickening, hyperpneumatization of paranasal sinuses and mastoides, cerebral peduncule atrophy, thalamic atrophy, lentiform nucleus hypoplasia.

\section{CONCLUSION}

cerebral hemiatrophy is a not usual syndrome in which mri is the procedure of choice with respect to assessment of the etiology and extent of cerebral parenchymal involvement in cerebral hemiatrophy. in our case the mri findings are suggestive of the antecedente of meningitis caused a important ischemia trought the right cerebral hemisphere.

\section{P010:19}

\section{BRAIN MRI FINDINGS IN AMITRIPTYLINE INTOXICATION}

\author{
Y. Durum $^{1}$, O. Dursun ${ }^{2}$, A. Aralasmak ${ }^{1}$, K. Karaali ${ }^{1}$ \\ ${ }^{1}$ Akdeniz University, Department of Radiology, Antalya, \\ Turkey; ${ }^{2}$ Akdeniz University, Department of Pediatrics, \\ Antalya, Turkey
}

\section{PURPOSE}

To present the brain MRI findings in a child with amitriptyline intoxication.

\section{METHODS}

A twenty-one-month-old girl who developed respiratory depression, lethargy and seizures after ingestion of 200-300 $\mathrm{mg}$ of amitriptyline was admitted to our pediatric intensive care unit. Mecanical ventilation and other symptomatic and supportive treatments were started immediately after the admission. On the follow up, the patient developed tachycardia and hypotension. As well, brain stem reflexes were absent and pupillary dilatation was observed. Patient was referred for brain MRI.

\section{RESULTS}

Brain MRI showed severe cerebral edema and tonsillar herniation. Bilateral and symmetric thalamic, basal ganglial, hippocampal and cortical lesions were also present. Restricted diffusion was also observed within these lesions as well as the whole supratentorial white matter.

\section{CONCLUSION}

To our knowledge, pathologic brain MRI findings in amitriptyline intoxication have not been presented in the literature. In this presentation the findings in our case are discussed and neurotoxicity mechanism of this drug is explained.

\section{PO10:20}

\section{MODERN IMAGING IN HMG-COA LYASE DEFICIENCY}

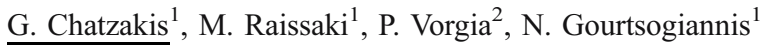

${ }^{1}$ Radiology Departement, University Hospital of Heraklion, Crete, Greece; ${ }^{2}$ Pediatrics Departement, University Hospital of Heraklion, Crete, Greece

Modern imaging in HMG-CoA lyase deficiency

\section{PURPOSE}

There are fewer than 100 reported cases with HMG-CoA lyase deficiency. White matter and basal ganglia ischemia, frontotemporal atrophy mimicking bilateral arachnoid cysts, megalencephaly and vascular insults resulting in unilateral atrophy have been reported.

To describe the MRI findings in HMG-CoA lyase deficiency. Materials and Methods

A 1-year-old male presented with hypoglycaemia, vomiting, seizures and became comatose. Laboratory test and an MRI scan of the brain were performed.

RESULTS

Hypoglycaemia and organic acids in urine were consistent with HMG-CoA lyase deficiency. MRI scan revealed hyperintensity and restricted diffusion at the lentiform nuclei, cerebral peduncles, quadrigeminal plate and superior cerebellar peduncles. There was prominence of the lateral ventricles and of the peripheral CSF spaces. Single voxel spectroscopy revealed an abnormal peak at $1.3 \mathrm{ppm}$. Follow-up MRI at 2 years of age revealed atrophy and restricted diffusion at thalami, lentiform and vestibular nuclei, 
inferior colliculi and cerebellar peduncles. Single voxel spectroscopy demonstrated the pathologic peak at $1.3 \mathrm{ppm}$.

\section{CONCLUSION}

Modern imaging may contribute to the diagnosis of metabolic disease, may aid in its differentiation from hypoglycaemia and may reveal progressive changes.

\section{P010:21}

CONGENITAL UNILATERAL ABSENCE OF INTERNAL CAROTID ARTERY: PATTERNS OF COLLATERAL FLOW AND ASSOCIATED FINDINGS IN THE PEDIATRIC AGE.

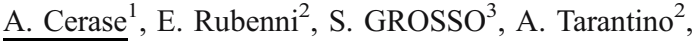
I. Vallone $^{1}$, P. Galluzzi ${ }^{1}$, S. Bracco ${ }^{1}$, C. Venturi ${ }^{1}$ UOC Neuroradiologia, Policlinico Santa Maria alle Scotte, Siena, Italy; ${ }^{2}$ Istituto di Radiologia, Università, Siena, Italy; ${ }^{3}$ Dipartimento Materno-Infantile, Policlinico Santa Maria alle Scotte, Siena, Italy

Congenital unilateral absence of internal carotid artery (CUAICA) is a rare developmental anomaly reported in $0.01 \%$ of population and up to $0,36 \%$ in angiographic studies. It is usually asymptomatic for collateral circulation, discovered incidentally in adulthood, and reported rarely in childhood.

The purpose of this scientific exhibit is to increase awareness to CUAICA in pediatric age, discuss embryology and collateral circulation, as well as possible clinical-neuroradiological associations in childhood, by describing findings at MRI, MRA, skull base HRCT and DSA observed in three female children with agenesis of ICA. At diagnosis, patient 1 was 5.5 year-old and presented with delayed neuromotor development, and seizures from age of 2 years, patient 2 was 12 day-old and presented with congenital anterior hypopituitarism, and patient 3 was 4-month-old and presented with left dropped lid, strabismus and hypoacusia. All patients underwent MRI/MRA and HRCT, patient 3 DSA. Clinical follow-up is 8.5 (patient 1), 4.5 (patient 2), and 2 (patient 3) years. Patient 1 underwent three MRI/ MRA follow-up examinations. CUAICA was right in patients 1 and 2 , and left in patient 3 (ipsilateral to symptomatic body side). Patterns of collateral circulation were fetal type (patient 1), adult type (patient 3 ), and through a trans-sellar vessel from contralateral ICA (patient 2). Patient 3 showed angiographic moyamoya. Other findings included aplastic adenohypophysis and ectopia of neurohypophysis (patient 2), and left mastoid hypoplasia (patient 3). Patient 1 had a chromosomopathy (translocation 9-4).

CUAICA is not an exceptional finding in the pediatric age. Most cases seem to be included in more complex syndromes, probably expression of a neural crest differentiation disorder or an abnormal development of sympathetic system. Its recognition at MRI/MRA is mandatory, since it may be key for correct explanation of pertinent physiopathology. Intracranial aneurysms increased prevalence, reported in association with CUAICA, is an indication for clinical and neuroradiological surveillance.

\section{PO10:22}

\section{CEPHALIC INDEX IN NORTH OF IRAN}

\author{
E. Bidabadi $^{1}$, M. Mashouf ${ }^{2}$ \\ ${ }^{1}$ Guilan University of Medical Sciences (Gums), Rasht, \\ Iran; ${ }^{2}$ Arya Hospital, Rasht, Iran
}

\section{INTRODUCTION}

With focusing on Guilanian's skull phenotypic appearance in the north of Iran, we expected that their skull were in brachycephalic range with measuring the cephalic index with plain skull x-rays. METHODS

This cephalometric survey was done on 190 healthy adults and 287 healty newborns skull radiographs. Maximal width and maximal length of skulls was measured and cephalic index was calculated as $\mathrm{MW} / \mathrm{ML}^{\times} 100$. Index between $75-80$ was assumed as mesocephalic, upper and lower results was assumed as brachy or dolicocephalic, respectively.

\section{RESULTS}

The mean cephalic index for Guilanian newborns was 78.368 (in the mesocephalic range) and for adults was 86.46 (in the brachycephalic range); $90 \%$ of adults and $40.1 \%$ of newborns had brachycephalic skulls, whereas $10 \%$ of adults and $35.9 \%$ of newborns had mesocephalic skulls.

\section{CONCLUSION}

According to cephalometric changes in Guilanian newborns and adults, it seems that environmental factors have more important rule than genetic factors for brachycephaly in the north of Iran.

\section{PO10:23}

\section{LISSENCEPHALY WITH CEREBELLAR HYPOPLASIA INITIALLY DIAGNOSED IN UTERO}

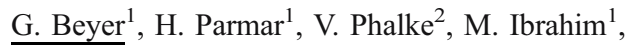
C. Van De Ven ${ }^{1}$

${ }^{1}$ University of Michigan, Ann Arbor, USA; ${ }^{2}$ Oregon Health \& Science University, Portland, USA,

\section{PURPOSE}

Lissencephaly with cerebellar hypoplasia $(\mathrm{LCH})$ is a rare type of congenital brain malformation with varying degree of lissencephaly associated with significant cerebellar underdevelopment. Currently six subtypes have been described in the literature, two of which are associated with mutations of LIS1, DCX and RELN genes, respectively. One of the rarest subtypes (type 6) involves lissencephaly, complete absence of corpus callosum and moderate to severe cerebellar hypoplasia. The purpose of this study is to illustrate the distinctive fetal and post natal MRI findings in a male patient with a rare type of lissencephaly with cerebellar hypoplasia-type $6 \mathrm{LCH}$ diagnosed in utero. We will also review the other subtypes of $\mathrm{LCH}$, as well as review other congenital malformations that can be confounders of LCH. 


\section{MATERIAL AND METHODS}

The material consists of gestational sonography at $185 / 7$ weeks, fetal MRI images at 21 weeks, and 1 day post natal brain MRI imaging at 38 $1 / 2$ weeks gestation.

\section{RESULTS}

Gestational sonography demonstrated non fusion of the frontal horns of the lateral ventricles, enlarged thalami with cerebellar flattening, and inferior vermis hypoplasia. Fetal MRI demonstrated similar findings with lissencephaly, complete absence of corpus callosum and cerebellar hypoplasia. Post-natal MRI findings were concordant with prior imaging depicting lissencephaly with severe cerebellar hypoplasia and complete absence of the corpus callosum.

\section{CONCLUSION}

The goal of this exhibit is to describe the distinctive imaging features on both fetal and post natal MRI of a rare subtype (type 6) of $\mathrm{LCH}$. We will describe other subtypes of LCH and briefly discuss the differential diagnosis.

\section{P010:24}

\section{PRENATAL BRAIN MRI IN THE EVALUATION OF GYRATION AND SULCATION—OWN EXPERIENCE}

A. Romaniuk-Doroszewska, M. Bekiesinska-Figatowska, H. Bragoszewska, A. Duczkowska

Institute of Mother And Child, Warsaw, Poland

\section{BACKGROUND}

Work in the centre having its own interdisciplinary team that makes the decisions concerning pregnancy management requires detailed knowledge of what is normal and abnormal on fetal MRI. PURPOSE

The purpose of this study is to create the atlas of fetal brain maturation on the basis of own material and equipment.

MATERIALS AND METHOD

Our experience results from over 300 prenatal MRI examinations that have been performed because of suspicion of anomalies on sonographic examination (US). Until the deadline of abstract submission we retrospectively reviewed 132 of them. The material chosen so far consists of 65 cases without any abnormalities of the central nervous system (CNS).

MRI was performed in 1.5 T GE Signa scanner. Single Shot Fast Spin Echo (SSFSE) was applied in T2 weighted images in axial, sagittal and coronal planes.

RESULTS

The gallery of MR images was created beginning with 19th week of gestation, showing details of brain structure, gyration and sulcation.

\section{CONCLUSION}

Own atlas of fetal brain maturation, showing the progress of sulcation and gyration week by week, is a very helpful tool in everyday practice.

\section{P010:25}

\section{CAUDATE NUCLEI VOLUME IN ADOLESCENTS \\ AND YOUNG ADULTS WITH PERSISTENT DEVELOPMENTAL STUTTERING}

\section{Adamczyk ${ }^{1}$, K. Szamburski ${ }^{2}$, T. Wolak $^{3}$,}

M. Bekiesinska-Figatowska ${ }^{4}$, A. Ciesielska ${ }^{5}$, K. Wagiel $^{4}$, P. Bogorodzki ${ }^{3}$, J. Walecki $^{6}$

${ }^{1}$ Department od Radiology, Central Clinical Hospital of the Ministry of Internal Affairs and Administration, Warsaw, Poland; ${ }^{2}$ Institute of Polish Language, University of Warsaw, Warsaw, Poland; ${ }^{3}$ Institute of Radioelectronics, Warsaw University of Technology, Warsaw, POLAND; ${ }^{4}$ Department of Diagnostic Imaging, Mother and Child Institute, Warsaw, Poland; ${ }^{5}$ Specialistic Psycho-Pedagogic Clinic TOP, Warsaw, Poland; ${ }^{6}$ Centre of Medical Postgraduate Education CSK MSWiA, Warsaw, Poland

\section{PURPOSE}

The anatomical and pathophysiological basis of persistent developmental stuttering (PDS) remains unknown. Some structural studies revealed few anomalies in cortical speech regions in PDS patients. It is suggested that possibly more important can be anomalies in subcortical nuclei, since they play a role in movement patterns, which seem to be impaired in stuttering people. The aim of the study was to find out if young people with PDS differ from healthy ones in caudate nuclei volumes and symmetry.

Methods. 24 patients with PDS (12 male and 12 female, age $22,9 \pm 4,91)$ and 20 healthy controls (10 male and 10 female, age $27,9 \pm 4,99)$ underwent head MRI. Each study subject was righthanded. T1-weighted gradient echo $1,5 \mathrm{~mm}$ thick images were obtained. Every brain was standardized. Volumes of caudate nuclei were measured by semiautomatic method.

RESULTS

Mean left caudate nucleus volume was $3,31 \pm 0,3 \mathrm{~cm} 3$ in PDS group and 3,39 $\pm 0,32 \mathrm{~cm} 3$ in control group. Mean right caudate nucleus volume was $3,36 \pm 0,27 \mathrm{~cm} 3$ in PDS group and $3,4 \pm$ $0,28 \mathrm{~cm} 3$ in control group. The values did not differ significantly between the groups. There was no asymmetry between left and right caudate nuclei in neither of groups observed.

CONCLUSIONS

The study did not reveal any premise that caudate nuclei could play a role in the pathogenesis of PDS.

\section{P010:26}

\section{MAGNETIC RESONANCE IMAGING (MRI) OF THE BRAIN OF PEDIATRIC PATIENTS PRESENTING TO OUR HOSPITAL WITH GLOBAL DEVELOPMENTAL DELAY}

\footnotetext{
A. ABD. Aziz ${ }^{1}$, C. Chin ${ }^{2}$, A. Abdullah ${ }^{2}$, S. Sivapunniam ${ }^{2}$, Y. Tan ${ }^{2}$

${ }^{1}$ International Islamic University Malaysia, Kuantan,

Pahang, Malaysia; ${ }^{2}$ Hospital Tengku Ampuan Afzan,

Kuantan, Pahang, Malaysia
} 


\section{PURPOSE}

Global developmental delay refers to a delayed in two or more important areas of development, which include motor skills, speech \& language, ability to learn new things and social \& personal skills. It can be due to a variety of possible causes but often the cause may not be determined. The aim is to illustrate the MRI brain findings of pediatric patients presenting to our hospital with global developmental delay.

\section{METHOD}

We retrospectively reviewed all MRI brain of paediatric patients aged up to 18 years who had MRI performed at our hospital from 1st July 2005 to 31st April 2007 for the assessment of global developmental delay. All the patients were clinically assessed by a Pediatrician. The MRI brain images with abnormal finding are illustrated.

RESULTS

A total of 34 patients; aged from 4 months to 12 years old had MRI brain performed during this period. Abnormal MRI brain was detected in 14 patients (41\%) comprising of 11 girls and 3 boys. Of these 14 cases, 3 showed syndromic features, 1 patient has Tuberous sclerosis and 1 patient has epilepsy.

The majority of MRI abnormalities detected were cerebral atrophy and encephalomalacia, while the remaining abnormality identified include a cerebral tumour, a retrocerebellar arachnoid cyst, gliosis, benign enlargement of the CSF (cerebrospinal fluid) space and thinned corpus callosum.

\section{CONCLUSION}

Neuroimaging particularly MRI brain is useful and is recommended to detect possible abnormality or damage to the brain tissues especially if other laboratory tests are negative. However they are not always diagnostic and will not be a substitute for a careful clinical assessment.

\section{P010:27}

\section{COMPARISON OF NEONATAL MRI EXAMINATIONS \\ WITH AND WITHOUT AN MR-COMPATIBLE \\ INCUBATOR: ADVANTAGES IN EXAMINATION \\ FEASIBILITY AND CLINICAL DECISION-MAKING.}

\author{
Z. Rona $^{1}$, K. Klebermass ${ }^{2}$, P. Brugger ${ }^{3}$, M. Weninger ${ }^{2}$, \\ A. Pollak ${ }^{2}$, D. Prayer ${ }^{3}$ \\ ${ }^{1} 1$ st. Dep. of Obstetrics and Gynacology, Semmelweis University, \\ Budapest, Hungary; ${ }^{2}$ Department of Neonatology and Intensive \\ Care, University of Vienna, Vienna, Austria; \\ ${ }^{3}$ Department of Radiology, University of Vienna, Vienna, Austria
}

\section{PURPOSE}

To assess the utility of an MRI-compatible incubator (INC) by comparing the frequency of and indications for MR-examinations in instable newborns with suspect central-nervous system (CNS) problems, done in periods of 18 months each, with and without INC. Additionally the respective expenditure of time, and amount of necessary sedatives were compared. The impact of MR-results on clinical management was determined.
Methods: In a retrospective study, we analyzed the clinical and radiological aspects of 138 neonatal MRI examinations during a 3 year period. Routine protocols including fast spin-echo T2weighted (w)sequences, axial T1w, Gradient-echo, diffusion sequences, and 3D T1 gradient echo sequences were performed routinely, angiography and spectroscopy were added in some cases. Diffusion-tensor imaging was done in $50 \%$ of the babies examined in the INC. Fetal sequences were done in infants younger than 32 gestational weeks.

\section{RESULTS}

The number of the examinations increased (39-99), while the mean age (46-38 weeks of gestational age) and weight (3365g-2791 g) decreased significantly with the use of the MR-compatible incubator. The mean imaging time $(30,1 \mathrm{~min})$ was equal in both periods, with a mean of 3 additionally performed sequences in the INC group. MR-based changes in clinical management were initiated in $66 \%$, while in $45 \%$ of cases the initial ultrasound diagnosis was changed or further specified. All infants received sedatives according to our anesthetic protocol preceding imaging, but a repeated dose was never necessary using the INC.

\section{CONCLUSIONS}

The use of an INC enables the MR-access of instable infants with suspect CNS problems, not only providing more accurate diagnoses but also having an impact on further management in more than the half of cases.

\section{PO10:28}

\section{MULTICENTER CLINICAL EVALUATION OF GADOBENATE DIMEGLUMINE FOR ENHANCED MR IMAGING OF CNS LESIONS IN CHILDREN}

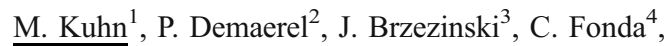
E. Cianciulli ${ }^{5}, \mathrm{P} \mathrm{Gao}^{6}$

${ }^{1}$ St. Johns Hospital, Springfield, USA; ${ }^{2}$ University Hospital K.U. Leuven, Leuven, Belgium; ${ }^{3}$ Helimed Diagnostic Imaging, Katowice, Poland; ${ }^{4}$ Anna Meyer Pediatric Hospital, Florence, Italy; ${ }^{5}$ Santobono Childrens Hospital, Naples, Italy; ${ }^{6}$ Tian Tan Hospital, Beijing, China

\section{PURPOSE}

Gadobenate dimeglumine (Gd-BOPTA) is a high relaxivity MR contrast agent that has been shown to result in improved enhancement of CNS lesions in adults. The application of this agent in children is less well characterized. We performed an open label multi-center phase-III study to evaluate the safety and efficacy of Gd-BOPTA in children $<18$ years old.

\section{METHODS}

Subjects between the ages of 2 to 17 years with known or highly suspected CNS disease referred for cranial or spinal MR examination were enrolled in the study at one of 6 centers worldwide. Safety monitoring included a history and physical examination, and pre- and post-dose monitoring of vitals signs, serial 12-lead electrocardiograms (ECG), and blood and urine samples. Gd-BOPTA (MultiHance, Bracco Imaging) was administered at a dose of $0.1 \mathrm{mmol} / \mathrm{kg}$ $(0.2 \mathrm{~mL} / \mathrm{kg})$ at a rate of $¡ \ddot{U} 2 \mathrm{~mL} / \mathrm{sec}$ followed by injection of normal 
saline. Pre-dose (T1wSE, T2wFSE, and FLAIR sequences) and postdose (T1wSE) images were evaluated for lesion enhancement, border delineation, and visualization of internal lesion morphology. All subjects were monitored for adverse events for 72 hours and followed up at 30 days to determine final diagnosis.

RESULTS

To date, 41 children with brain or spinal disease have been studied. Gd-BOPTA was well tolerated by all subjects with no clinically significant or serious adverse events reported. No clinically meaningful changes in laboratory or ECG parameters have been observed during the study period. In patients with enhancing lesions, assessment of lesion border delineation, definition of disease extent, visualization of lesion internal morphology and lesion contrast enhancement were considered good to excellent in all subjects. In subjects with enhancing brain lesions, Gd-BOPTA provided information complimentary to that found on pre-dose images.

CONCLUSIONS

Our experience suggests that Gd-BOPTA is a safe and effective MR contrast agent for the evaluation of CNS lesions in children.

\section{Head and Neck}

\section{P011:1}

\section{DINAMIC CE FAT SUPPRESSED MRI OF THE ORBIT IN PATIENTS AFFECTED BY ORBITOPATHY AND TREATED WITH AN EXPERIMENTAL THERAPY}

\author{
M. Conti ${ }^{1}$, A. Achene ${ }^{1}$, C. Urigo ${ }^{1}$, S. Frau ${ }^{1}$, R. Lai ${ }^{1}$, \\ L. Canalis ${ }^{1}$, M. Palermo ${ }^{1}$, G. Canalis ${ }^{2}$ \\ ${ }^{1}$ Istituto di Scienze Radiologiche, Sassari, Italy; ${ }^{2}$ Istituto di \\ Patologia Medica, Sassari, Italy
}

\section{PURPOSE}

To evaluate functional and quantitative parameters of extraocular muscles $(\mathrm{EOM})$ in patients affected by thyroid orbitopathy who underwent an experimental pharmacologic treatment protocol.

\section{MATERIALS \& METHODS}

From March 2005 to October 2006, 30 patients underwent MR examinations (Philips Intera Achieva $1.5 \mathrm{~T}$ ) before (group A1) and after (group A2) the therapy and their data were compared to 10 healthy subjects (group B). On the four rectus muscles coronal and axial diameters and the areas at the largest extent of the muscle belly were evaluated on T1-weighted images as well as peak enhancement ratio (rP) versus temporalis muscle and maximum intensity peak in time-intensity curves were calculated on a dynamic CE fat-suppressed sequence.

Statistical analyses were perfomed by $\mathrm{t}$ student test between group $\mathrm{B}$ and $\mathrm{A} 1$ and $\mathrm{A} 1$ and $\mathrm{A} 2$.

\section{RESULTSHY}

Diameters and areas of each EOM were bigger in group A1 than B $(\mathrm{p}<0.001)$; peak enhancemt ratio versus temporalis muscle was higher in group $B(p<0.001)$ and maximum intensity peak was faster in group $B(p<0.001)$. After therapy the differences were not statistically significant $(\mathrm{p}>0.005)$ for either quantitative and functional parameters even if a functional improvement was described.

\section{CONCLUSION}

Dynamic CE fat-suppressed sequence provides optimal parameters concerning functional abnormalities correlated with the clinical course of the disease.

They are useful in evaluating both effectiveness and dose-related changes of extraocular muscles involvement in thyroid orbitopathy. Furthermore this new metil-prednisone-based therapy is emerging in clinical practice so that it needs a safe and appropriate confirmation which can be achieved only by evaluation of clinical data as well as muscles functional and quantitative analysis performed by MR study.

\section{PO11:2}

\section{SPONTANEOUS CSF FISTULA OF THE ANTERIOR SKULL BASE CAUSING MASSIVE PNEUMOCEPHALUS DETECTED WITH A 3D T2-WEIGHTED SINGLE-SHOT FAST SPIN-ECHO SEQUENCE (3D-EXPRESS)}

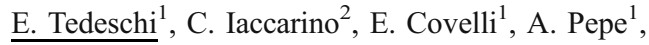
F. D'Agostino ${ }^{1}$, M. Barretta ${ }^{1}$, A. Rapanà ${ }^{2}$, G. Belfiore ${ }^{1}$

${ }^{1}$ Diagnostic Imaging Dept. - AORN S.Anna e S.Sebastiano, Caserta, Italy; ${ }^{2}$ Neurosurgery Unit - AORN S.Anna e S. Sebastiano, Caserta, Italy

\section{PURPOSE}

To report the usefulness of a heavily T2-weighted 3D single-shot fast spin-echo sequence with half-Fourier analysis (EXPRESS: extended-phase conjugate-symmetry rapid spin-echo-Marconi Medical Systems), routinarily used for imaging the internal ear and internal auditory canal structures, in the detection of a suspect CSF fistula of the anterior skull base.

\section{METHODS}

A 32 year old caucasian female with no history of trauma or intracranial surgery, presenting at the Emergency Room with rhinorrhea and severe headache, showed massive penumocephalus in all the CSF spaces surrounding the brainstem and in the supratentorial ventricular system at $\mathrm{CT}$, but no skull base fracture. Contrast-enhanced brain MR was performed at $1.5 \mathrm{~T}$ (Eclipse, Marconi-Philips) to evaluate the sellar region and also using a coronal thin-slice $(0.8 \mathrm{~mm})$ 3D-EXPRESS sequence (TR: $5000 \mathrm{~ms}$, TE: $126 \mathrm{~ms}$, ETL: 136, Interecho Spacing: $8.5 \mathrm{~ms}$, FOV: $16 \mathrm{~cm}$, FA: $90^{\circ}$, Matrix: $256 \times 256$, TA: $4.00 \mathrm{~min}$ ), covering the anterior skull base, in order to detect the possible site of CSF leakage.

RESULTS

Beside pneumocephalus, conventional MR sequences showed no pituitary gland pathology and unremarkable brain findings. 3DEXPRESS coronal thin slices allowed detailed evaluation of the floor of the sphenoid sinus and of the posterior ethmoid cells, with excellent conspicuity of the CSF signal which could be followed from the left parasellar region down to the left nasal cavity. Based on the MR findings, sinus packing was performed via a trans-nasal approach, with rapid resolution of the symptoms. 


\section{CONCLUSIONS}

Express sequence may be useful for non-invasive detection of CSF fistulae of the anterior skull base even when massive pneumocephalus is present, eliminating the need of CT-cisternography.

\section{P011:3}

\section{FAT SUPPRESSION MODEL AT THE NECK REGION IN MRI}

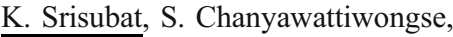

C. Charoenwikrom, C. Saritcharoenkha, C. Bunsong

Prasat Neurological Institute, Bangkok, Thailand

One factor that affects to fat suppression is the homogeneity of magnetic field. Neck is the area that composes of many different tissues and is affected by the orientation of the fat-air interface. These problems cause field inhomogeneity and susceptibility artifact. Radiological technologist has a role to reduce the susceptibility artifact by placing filling material such as water bag or Sat Pad at the neck area. The problem of water bag is leakage of water and difficult placing around the neck. The problem of Sat Pad is very expensive and cannot find in Thailand. The Purpose of the study is the production a suitable model to solve the problem and can be produced in Thailand. Methods: Producing fat suppression model by filling ultrasound gel in several sizes of thin plastic sacs and sealed it. Besides the gel model we used cold hot pack in this study too. Compare these two models to the present technique of water bag by experiment in 40 samples in coronal T1 fat suppression with map shim and auto shim adjustment techniques. Result: The study found that the model made from ultrasound gel filled in the thin plastic sac can be used easily because it can move along any shape and less leakage. Our study reveals that the ultrasound gel model can suppress the fat signal better than water bag or cold hot pack,especially at the supraclavicular fossa which is a fat suppression difficult area (pvalue $<0.05$ ). The study also shows that auto shim adjustment for magnetic field should be practiced to get high homogeneity and get more fat suppression. Conclusion: Ultrasound gel model can be used for fat suppression more than water bag and cold hot pack. Besides this,ultrasound gel model can be used in different sizes of necks and can be improved the technique at Prasat Neurological Institute and others. The cost is not too expensive.

\section{P011:4}

\section{BRACHIOCEPHALIC VEINS REMODELLING IN CASES OF HEMODYNAMIC SIGNIFICANT EXTRAVASAL COMPRESSION}

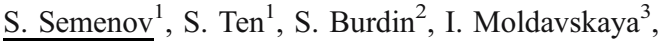 \\ I. Sizova ${ }^{1}$ \\ ${ }^{1}$ Cardiology Centre, Kemerovo, Russia; ${ }^{2}$ Clinical \\ Diagnostic Centre, Kemerovo, Russia; ${ }^{3}$ Railway Regional \\ Hospital, Kemerovo, Russia
}

The goal of the investigation was brachiocephalic veins remodelling process studying in cases of long-term obstruction by means of non-invasive visual methods.

Material and methods: The Color Doppler Triplex scanning and 2D TOF MR-venography were performed for examining of 56 patients (average age 43,2) with the venous encephalopathy resulted from hemodynamic significant extravasal compression of internal jugular and innominate veins.

\section{RESULTS}

As a rule the cross-section and the blood flow velocity of compressed internal jugular vein were significantly reduced 3-4 times if compared to the opposite enlarged vein. The enlargement was also noted at other neck venous collectors (external and anterior jugular veins, vertebral veins and plexus, posterior servical and subcutaneous veins).

The consequence of the neck venous collectors compensation enlargement was determined. In an acute period in the case of unilateral internal jugular vein compression the contralateral one was enlarged. If the compression continued ipsilateral external jugular vein enlarged in the subacute period with the contralateral external jugular vein enlargement following.

In the late subacute and chronic periods vertebral veins crosssection and blood flow velocity enlargement was noted as well.

Lateral venous sinuses and/or internal jugular veins thrombosis was diagnosed at 13 patients who had been monitored within 25 years as regards brachiocephalic veins extravasal compression on the same side. This was accompanied by their clinical status worsening.

\section{CONCLUSION}

Compensation neck venous vessels remodelling process is typical for all the cases of unilateral brachiocephalic veins hemodynamic significant compression from the viewpoint of chronology and consequence as the result of autoregulation and blood flow redirection of cerebral circulation.

\section{P011:5}

DISSECTION OR NO DISSECTION-ROLE OF 3D TOF MRA WITH PROXIMAL AND DISTAL PRE SATURATION PULSE

V. Sawlani, J. Sparkes, N. Powell, T. Edwards, J. Martin

Morriston Hospital, Swansea, United Kingdom

\section{PURPOSE}

Diagnosis of vertebral artery dissection represents an important challenge. Flow related enhancement in the vertebral venous plexus on $\mathrm{T} 1 \mathrm{~W}$ fat saturation sequence and MR angiography could mimic an intramural haematoma and can be misinterpreted as dissection. The role of distal and proximal pre saturation pulse with 3D TOF MRA is evaluated to distinguish vertebral venous plexus form vertebral artery dissection.

\section{MATERIALS AND METHODS}

A 19 year young rugby player presented with neck pain and vertigo after a blunt trauma during rugby match. His MRI examination with fat saturation $\mathrm{T} 1 \mathrm{~W}$ axial sequence showed a semi lunar bright signal surrounding the narrowed lumen of right 
vertebral artery. Axial source images and MIP images of 3D TOF MRA showed loss of signal in right vertebral artery (V3 segment) between $\mathrm{C} 1$ and $\mathrm{C} 2$ vertebrae. Axial source images of the 3D TOF MRA with distal venous pre saturation pulse and proximal arterial pre saturation pulse showed no intramural haematoma or dissection.

\section{RESULTS}

The semi lunar hyper intensity in the periphery of a suspected dissected vertebral artery on $\mathrm{T} 1 \mathrm{~W}$ fat saturated axial spin echo MR images due to flow related enhancement in the vertebral venous plexus, could be misinterpreted as an intramural haematoma. The distal venous pre saturation pulse would saturate the cranio caudal flow in venous plexus and proximal arterial pre saturation pulse would saturate the arterial luminal flow. Hence in case of no dissection as seen in our case there would be no hyper intense signal seen at all. Where as in the case of true dissection intramural haematoma (methaemoglobin) would still be seen as hyperintense.

\section{CONCLUSION}

In suspected cases of vertebral artery dissection, the use of proximal and distal pre saturation pulses with 3D TOF MRA may help distinguish blood flow in the vertebral venous plexus from vertebral artery dissection.

\section{P011:6}

\section{TIME RESOLVED 3D CONTRAST ENHANCED MRA} OF CRANEAL AND CERVICAL VASCULAR DISEASES.

I. Saralegui Prieto $^{1}$, A. Cabrera Zubizarreta ${ }^{1}$, I. Vicente Olabarria ${ }^{1}$, E. Astigarraga Aguirre ${ }^{1}$, R. Ituarte Uriarte $^{2}$

${ }^{1}$ Osatek S.A., Galdakao, Spain; ${ }^{2}$ Dep. Neuroradiology.

Hospital Cruces, Bilbao, Spain

\section{PURPOSE}

To asses the usefulness of a time resolved $3 \mathrm{~d}$ mra sequence as a first step examination in the evaluation of vascular craniocervical pathology.

\section{METHODS}

We have studied 28 patients with craniocervical vascular disease, 7 with craneal arteriovenous malformations (avm), 7 with carotid cavernous fistula (ccf), 3 with intracranial dural fistula (dvaf), 4 tumours (1 carotid paraganglioma, 1 jugulotympanic paraganglioma, a typical and an atypical meningioma) and 7 patients with pathology in the thoracic outlet (1 jugulosubclavian fistula, 1 jugulovertebral fistula, 2 subclavian steal syndromes).

Examinations were performed in a $1.5 \mathrm{t}$ system with a 8-channel head coil. A 3d gradient echo sequence with the following parameters was adquired: tr/te $2.84 / 1.17, \alpha 25^{\circ}$, bandwidth $890 \mathrm{hz} /$ pixel, fov 243/62.5, $256 \times 125$ matrix, voxel size $1.2 \times 0.9 \times 0.6$, parallel imaging with grappa ( 2 directions) algorithm and a temporal resolution of $1 \mathrm{~s}$ per data set with an adquisition time of $38 \mathrm{~s}$. A 3-4 ml bolus of either gadodiamide or gd-bopta was administered using power injector. RESULTS

Five left ccf, 1right ccf and 1 bilateral ccf were demonstrated. Superior ophtalmic vein was enlarged in 6 cases and normal in
1 case. Mav showed anterior circulation feeders in 1 case, posterior feeders in 5 cases and both anterior and posterior in 1 case. Two dvaf were infratentorial with drainage to transverse and sigmoid sinus and 1 was frontoparietal with drainage to the superior longitudinal sinus.

Mra demonstrated most of the arterial feeders and the venous drainage. The hemodynamic pattern was correctly depicted in cases of davf, ccf, cervical arteriovenous fistulas, and mav, (considering digital subtraction arteriography as the gold standard). The mra matched the surgical findings in two tumoral cases.

\section{CONCLUSION}

Time resolved $3 \mathrm{~d}$ mra can be considered a useful complementary tool in the hemodynamic evaluation of craneal and cervical vascular pathology, prior to dsa or surgery

\section{PO11:7}

\section{THE NEAR OCCLUSION IN THE CAROTID ARTERY: HOW TO ANALYZE IT BY USING MDCTA}

\section{Saba, G. Mallarini}

Policlinico Universitario di Monserrato, Cagliari, Italy

\section{PURPOSE AIM}

1) To describe the normal anatomy of carotid artery and atherosclerotic carotid artery. 2) To review the concept of "near occlusion". 3) To review the CTA technical parameters to be used and which techniques can be used including the visualization difference between maximum intensity projection (MIP), multi-planar reconstruction (MPR), curved planar reformation (CPR) and volume rendering (VR) for the study of "near occlusion" carotid artery.

\section{CONTENT ORGANIZATION}

1) Normal anatomy of carotid artery and atherosclerotic carotid artery. 2) Physiopathology and implications of near occlusion. 3) To review the CTA technical parameters to be used and which techniques can be used including the visualization difference between maximum intensity projection (MIP), multi-planar reconstruction (MPR), curved planar reformation (CPR) and volume rendering (VR) for the study of "near occlusion" carotid artery. SUMMARY

Near occlusion stenosis indicates a severe carotid bulb stenosis with subsequent subtle decrease in size of the distal ICA lumen. Detection and precise interpretation of near-occlusion are important to plan a proper diagnosis and treatment. The use of post processing tool is helpful in the confirmation of "near occlusion" and in particular the CPR post-processing.

\section{P011:8}

THE ANALYSIS OF CAROTID SIPHON: THE USE OF CT IS A FEASIBLE MODALITY?

L. Saba, G. Mallarini

Policlinico Universitario di Monserrato, Cagliari, Italy 


\section{PURPOSE}

1)To describe the normal anatomy of carotid siphon, 2) To understand physiopathology of carotid siphon stenosis 3) To review the CTA technical parameters to be used and which postprocessing techniques can be employed.

CONTENT ORGANIZATION

Normal anatomy of carotid siphon, 2) physiopathology of carotid siphon stenosis 3) To review the CTA technical parameters to be used and which post-processing techniques can be employed. 4) Visualization difficulties, artefacts and pitfalls in the study of carotid siphon.

\section{SUMMARY}

Carotid artery stenting or carotid endarterectomy are widely used in the treatment of carotid artery stenosis, but should be avoided when there is carotid siphon stenosis. So, the correct identification of pathology at this level is very important. Multi-detector-row CT angiography shows a great efficacy in the study of carotid siphon. Post processing procedures rarely add relevant informations in comparison with axial images.

\section{P011:9}

\section{A PICTORIAL REVIEW OF INTRINSIC BRAINSTEM PATHOLOGIES AFFECTING ADULT AND PEDIATRIC POPULATION}

S. Munusamy, G. Deib, H. Stockley, C. Soh,

A. Herwadkar, R. Laitt, D. Hughes, I. Turnbull, J. Gillespie, S. Forbes ${ }^{1}$ Department of Neuroradiology, Hope Hospital, Salford, Manchester, United Kingdom

\section{PURPOSE}

To demonstrate that there is a wide variety of diseases that affect the brainstem, not all tumours, and not all fatal. Many are reversible and some are curable.

A pictorial review is presented showing numerous examples across the pathological spectrum affecting both adult and pediatric population

METHODS

MR images of both adult and pediatric patients were retrospectively reviewed covering the interval between 1993 and 2007 (last 13 years) in the Regional Infectious Disease Centre, North Manchester General Hospital, Greater Manchester Centre for Neurosciences, Hope Hospital, Salford, and Royal Manchester Children's hospital, Pendlebury, Manchester, United Kingdom. RESULTS

The brainstem is home to a wide range of diseases including neoplasms, infections, inflammatory conditions and vasculitides. Common and rare pathologies affecting both adults and pediatric population are discussed, including those affecting the cranial nerve nuclei (particularly CN V and VII). Early recognition is essential in many entities to allow for treatment and minimize disability. CONCLUSION

Radiologists need to be aware that not all intrinsic brainstem lesions are neoplastic and not all neoplasms are malignant. Using imaging characterisation and careful clinico-radiological correlation will often yield the diagnosis in all age groups.

\section{PO11:10}

\section{BILATERAL CONGENITAL COLOBOMATOUS CYSTS AND MICROPHTALMIA}

\author{
$\underline{\text { F. Kendiroglu }}^{1}$, K. Karaali ${ }^{1}$, E. Mihci ${ }^{2}$, Y. Duranoglu ${ }^{3}$, \\ G. Arslan ${ }^{1}$ \\ ${ }^{1}$ Akdeniz University, Department of Radiology, Antalya, Turkey; \\ ${ }^{2}$ Akdeniz University, Department of Pediatrics, Antalya, TURKEY; \\ ${ }^{3}$ Akdeniz University, Department of Ophthalmology, Antalya, \\ Turkey
}

\section{PURPOSE}

Colobomatous cysts of the orbits are extremely rare. We are reporting a case of congenital bilateral ocular colobomatous cysts with bilateral microphtalmia.

\section{METHODS}

A six-month-old female patient followed up with bilateral blepharospasm, microphtalmia and developmental delay in anterior ocular segment of the orbits was referred to our MRI unit to evaluate the additional intraocular and intracranial anomalies. RESULTS

Ocular and cranial MRI and multislice CT are performed. MRI and coronal CT scans demonstrated bilateral intraocular, well circumscribed, rounded cystic lesions in the inferior sides of the orbits with bilateral microphtalmia. The cystic lesion on the right was larger than the one on the left and also was indenting the orbital wall. The right optic nerve was thinner than the left. There was no central nervous system abnormality.

\section{CONCLUSION}

Colobomatous orbital cyst with microphtalmia is a rare anomaly occuring during embryogenesis. We present the MRI and CT findings of a case of congenital bilateral colobomatous cysts with microphtalmia.

\section{P011:11}

INFRANUCLEAR FACIAL NERVE PALSY: CORRELATION OF CLINICAL AND MRI FINDINGS

V. Katsaros ${ }^{1}$, P. Lampropoulou ${ }^{1}$, M. Mitropoulou ${ }^{1}$, A. Nikolaou $^{1}$, C. Samara ${ }^{2}$, C. Drossos ${ }^{2}$

${ }^{1}$ IKA Oncology Hospital, Department of CT and MRI, Athens, Greece; ${ }^{2}$ Athens General Hospital, Department of Modern Imaging Modalities, Athens, Greece

\section{PURPOSE}

The aim of this study was to evaluate 3D CISS, unenhanced and contrast en-hanced SE high-resolution thin-slice T1W images for the diagnosis of neoplastic, vascular, inflammatory and miscellaneous lesions causing facial nerve palsy. 


\section{PATIENTS AND METHODS}

112 MR examinations were performed on a total of 106 patients (67 males, 39 females; aged 6-77 years, mean age 48 years) using a 1.5 T MR unit. A GRE 3D CISS sequence and a SE highresolution thin-slice T1W sequence in axial and coronal plane with and without contrast medium administration (gadolinium-DTPA, $0.2 \mathrm{mmol} / \mathrm{kg}$ body weight) were obtained. Correlation with clinical and laboratory findings followed.

\section{RESULTS}

No evidence of significant findings was observed in 40 patients with Bell's palsy. Pathological enhancement in SE high-resolution thin-slice T1W sequence was found in the following lesions: 1) viral: herpes virus complex, 4, Ramsay-Hunt syndrome, 2, 2) bacterial: pyogenic medial ear inflammation, 5, external ear inflammation, 2, 3) immunological reaction: Guillain-Barré, 1, multiple sclerosis, 4, Melkerson-Rosenthal syndrome, 1, 4) medical: diabetes mellitus, 2, sarcoidosis (Heerfordt syndrome), 1, 5) neoplastic: schwannoma, 1, acoustic neuroma, 15, meatal meningioma, 1, meningeal carcinomatosis, 5 (breast carcinoma, 3, lung carcinoma, 2), 6) trau-matic: post-surgery, 2, head injury, 10, 7) miscellaneous: cholesteatoma, 7, epidermoid, 3 . Results of $3 D$ CISS-filling defects were found with the following lesions: schwannoma and acoustic neuroma, 16; meningioma, 1; meningeal carcinomatosis, 5 (breast carcinoma, 3, lung carcinoma, 2). CONCLUSION

These results suggest that unenhanced and contrast enhanced highresolution thin-slice SE T1W and 3D CISS are complementary MR imaging modalities. In correlation with clinical and laboratory findings they establish the correct diagnosis in facial nerve palsy.

\section{P011:12}

\section{PRIMARY LOCALIZED ORBITAL AMYLOIDOSIS}

V. Katsaros ${ }^{1}$, P. Brestas ${ }^{1}$, A. Roumeliotis ${ }^{2}$,

D. Mountzouris ${ }^{1}$, A. Katsarou ${ }^{3}$, F. Skopouli ${ }^{4}$

${ }^{1}$ Attica Health Care, MRI Unit, Pallini, Greece; ${ }^{2}$ Ygheia Hospital, Department of Ophthalmology, Athens, Greece;

${ }^{3}$ Red Cross Hospital, Department of Radiology, Athens, Greece; ${ }^{4}$ Euroclinic of Athens, Department of Rheumatology, Athens, Greece

\section{PURPOSE}

Amyloidosis refers to a heterogeneous group of disorders associated with deposition of chemically distinct fibril proteins. Isolated orbital amyloidosis is a rare condition and requires systemic examination. a case of amyloid deposit in the orbit whose systemic investigation has been negative is reported. METHODS: A 56-year-old woman presented to the eye clinic with left-sided orbital mass, mild exophthalmos with downward ocular displacement, and ptosis. No systemic involvement was noted by systemic workup and it confirmed the primary orbital amyloidosis.

RESULTS

The patient was subject to full clinical examination, laboratory examinations, orbital echography, magnetic resonance imaging and to- tal body computed tomography scans, rectal mucosa, and temporal artery biopsies. It was necessary to exclude systemic amyloidosis. CONCLUSIONS

Orbital amyloidosis is a very rare disease. It should be considered in the diagnosis of patients with ptosis and exophthalmos. The treatment usually consists of surgical removal of the amyloid mass and follow-up for a likely local recurrence.

\section{PO11:13}

\section{MR DACRYOCYSTOGRAPHY: NORMAL AND PATHOLOGIC FINDINGS}

\section{K. Karaali ${ }^{1}$, Y. Durum ${ }^{1}$, B. Agirdir ${ }^{2}$, C. Apaydin ${ }^{3}$}

${ }^{1}$ Akdeniz University, Department of Radiology, Antalya, Turkey;

${ }^{2}$ Akdeniz University, Department of ENT \& HNS, Antalya, Turkey;

${ }^{3}$ Akdeniz University, Department of Ophthalmology, Antalya, Turkey

\section{PURPOSE}

To present normal and pathologic findings in MR dacryocystography METHODS

MR dacryocystography has been performed in our institution for the last three years in patients with suspected lacrimal outflow system pathology. In this technique, $0.5 \%$ of Gadolinium solution is instilled into the conjunctival cul de sac while the patient is lying in the magnetic resonance gantry. Total five drops of the solution is instilled to each eye. Imaging is performed immediately after the instillation. T1-weighted gradient-echo (T1-FFE) sequences in coronal plane are obtained for the evaluation of nasolacrimal canalicular system.

RESULTS

In this presentation, normal anatomy and MR dacryocystographic appearances of the nasolacrimal canalicular system are demonstrated. As well, examples of pathologic findings such as unilateral and bilateral stenosis, occlusion, mucocele formation are given. Postoperative appearances after dacryocystorhinostomy are also shown. CONCLUSION

Several different imaging methods are available to image the nasolacrimal system. MR dacryocystography is a suitable method for the evaluation and has several advantages over the other techniques. Examples of normal and abnormal appearances in this technique are given in the presentation.

\section{P011:14}

\section{CLINICAL, RADIOLOGICAL AND EPIDEMIOLOGICAL REVISION OF BILATERAL CAVERNOUS HEMANGIOMAS OF THE ORBITS}

A. Paonessa ${ }^{1}$, N. Limbucci ${ }^{2}$, F. Quaranta Leoni ${ }^{3}$, A. Splendiani $^{2}$, M. Gallucci ${ }^{2}$

${ }^{1}$ Dept of Neuroradiology, Loreto Nuovo Hospital, Naples, Italy; ${ }^{2}$ Dept of Neuroradiology, University Hospital, L'Aquila, Italy; ${ }^{3}$ Dept. of Ophthalmology, University Campus Bio-Medico, Rome, Italy 


\section{INTRODUCTION AND AIMS}

14 patients, 3 of which presenting bilateral orbital lesions with CT and MRI findings typical of cavernous haemangiomas, had been studied

Materials and method: 14 consecutive patients ( 8 females and 6 males) with orbital cavernous haemangiomas underwent orbital axial and coronal CT and MRI.

MRI of the brain was performed on a 1.5-T system. Non-enhanced T1-w SE were obtained in the axial planes. PD and T2-w TSE, and FLAIR were performed in the axial and coronal planes. MRI of orbits was performed with fat-sat T2-w TSE images, and T1w SE images in the axial and coronal planes, section thickness of $3 \mathrm{~mm}$. Gd-enhanced fat-sat T1-w were obtained in the axial and parallel to the optic nerve planes with section thickness of $3 \mathrm{~mm}$. RESULTS AND CONCLUSION

All patients presented with well-defined, rounded, extraocular masses, intraconal, with smooth contours, bilateral in 3 cases $(21,4 \%)$. In these cases the symptoms were unilateral, and interested only the orbit with the larger mass. 13 masses appeared isointense on T1, 2 were hypointense, and 2 heterogeneous. On T2, 12 were hyperintense, 3 were heterogeneous, 1 isointense, and 1 hypointense. C.E. was inhomogeneous in 5, homogeneous and intense in 12 cases. The size ranged from 3 millimeters up to 12 millimeters (average 14.57). In all patients only the symptomatic lesion was surgically removed and confirmed histologically. Cavernous hemangioma is considered in literature to be almost always unilateral, multiple or bilateral location representing less than $4.7 \%$, while in our experience, this incidence was much higher $(21.4 \%)$.

About this apparent incongruence, the main reason is related to technological advances. Our results could be related to carelessness of radiologists to inspect the other orbit for possible lesions, and thus invites to put major attention to always explore both orbits for eventual bilateral lesions.

\section{P011:15}

\section{PROGNOSTIC FACTORS OF NPC INVESTIGATED BY MR IMAGING AND THE VALUE OF MR IMAGING IN LOCAL OUTCOME AND DISTANT METASTASIS}

\author{
C. Chen ${ }^{1}$, J. Ling ${ }^{2}$, J. Chai ${ }^{1}$, C. Chiang ${ }^{1}$, \\ W. Chen ${ }^{1}$, H. Hung ${ }^{1}$ \\ ${ }^{1}$ Department of Radiology, Taichung Veterans General Hospital, \\ Taichung, Taiwan; ${ }^{2}$ Department of Radiation Oncology, Taichung \\ Veterans General Hospital, Taichung, Taiwan
}

\section{PURPOSE}

To retrospectively assess the prognostic meaning of tumor characteristics depicted on pretreatment MRI for local outcome, neck recurrence and distant metastasis in NPC.

\section{METHODS}

We studied 129 NPC patients with MRI before neoadjuvant chemotherapy and radiotherapy. The involvement structures, neck lymphadenopathy, primary tumor volume (PTV) and the greatest dimension of neck lymph node were evaluated in detail by MRI. The median follow-up to last contact of patient was 39 months, with a range of 11 to 78 months. The cutoff date for analysis was October 2005. All surviving patients had been followed for a minimum of 2 years.

\section{RESULTS}

The primary tumor staging (UICC) was T1 in 23, T2a in 19, T2b in 18, T3 in 41 and T4 in 28. Neck lymph node staging was N1 in $28, \mathrm{~N} 2$ in $69, \mathrm{~N} 3 \mathrm{a}$ in 27 and N3b in 3. The PTV was ranged from 1.2 to $77.5 \mathrm{cc}$ (median, $19.2 \mathrm{cc}$ ), and the greatest dimension of neck lymph node was ranged from 0 to $12.3 \mathrm{~cm}$. Totally, there were 43 cases of treatment failure which were nasopharyngeal local and/or neck recurrence, and distant metastasis. In the 11 local recurrent patients, primary tumor stagings were $\mathrm{T} 2 \mathrm{~b}$ in $1, \mathrm{~T} 3$ in 2 and T4 in 8 and the average PTV was $24.3 \mathrm{cc}$. In the 36 distant metastasis patients, the primary tumor stagings were $\mathrm{T} 1$ in $1, \mathrm{~T} 2 \mathrm{a}$ in 2, T2b in 4, T3 in 10 and T4 in 19. The stagings of neck nodal metastasis were 6 in N1, 11 in N2, 17 in N3a and 2 in N3b. The PTV was ranged from $7.4 \mathrm{cc}$ to $77.5 \mathrm{cc}$ and the greatest dimension of neck lymph node was ranged from $1.8 \mathrm{~cm}$ to $12.3 \mathrm{~cm}$.

\section{CONCLUSION}

Pre-treatment MR imaging of NPC is useful in evaluating the primary tumor invasion and neck lymphadenopathy, and predicts local control and/or distant metastasis. The local recurrence appeared more readily in high $\mathrm{T}$ stage- $\mathrm{T} 3$ and $\mathrm{T} 4$. Distant metastasis mostly occurred in patients of T3 and T4 in T staging and of $\mathrm{N} 3$ in $\mathrm{N}$ staging, or being with bulky neck node involvement.

\section{P011:16}

\section{IMAGING OF THE SUPRAHYOID CAROTID SPACE:} A PICTORIAL REVIEW

N. Chaudhary, S. Connor

King's College Hospital, London, United Kingdom

Knowledge of the contents of the suprahyoid carotid space and its anatomy facilitates localisation and diagnosis of masses in this region.

We describe and illustrate the normal CT and MR anatomy of the suprahyoid carotid space. The characteristics of lesions arising from this location and differentiation from mass lesions in adjacent spaces will be discussed. An approach to the imaging analysis of a suprahyoid carotid space lesion will be outlined. The radiological features of vascular lesions (internal carotid ectasia, aneurysm, pseudoaneurysm, dissection, jugular vein thrombosis), inflammatory lesions, benign tumours (carotid body tumour, glomus jugulare, glomus vagale, schwannoma, neurofibroma, meningioma) and malignant invasion will be described and illustrated. 


\section{P011:17}

\section{WELKER'S BASAL ANGLE IN THE PLAIN SKULL X-RAYS IN THE NORTH OF IRAN}

E. Bidabadi ${ }^{1}$, M. Mashouf ${ }^{2}$

${ }^{1}$ Guilan University of Medical Sciences (Guns), Rasht, Iran; ${ }^{2}$ Arya Hospital, Rasht, Iran

\section{INTRODUCTION}

With focusing on Guilanian's skull phenotypic appearance and brachycephaly in the north of Iran, we expected that their Welker's basal angle measuring with plain skull $\mathrm{x}$-rays was different with normal range (about $134^{\circ}$ ).

\section{METHODS}

This craniometric survey was done on 180 healthy adult skull radiographs. Basal angle of skulls was measured; angle between $123^{\circ}-143^{\circ}$ was assumed as normal, upper and lower results was assumed as platybasia or basilar kyphosis, respectively.

RESULTS

Normal basal angle was seen in $87.78 \%$ of cases, whereas basilar kyphosis was seen in $12.22 \%$ of cases; no platybasia has been observed in this study. The basal angle range was $115^{\circ}-139^{\circ}$, with mean angle of $128.75^{\circ} \pm 5.35$; mean of angle was $128.66^{\circ} \pm 5.44$ and $129.53^{\circ} \pm 4.59$ for male and female cases, respectively. There was not statistically significant difference between basal angle among different adult age groups.

\section{CONCLUSION}

In spit of brachycephalic skulls in the Guilanian adults, it seems that their basal angles were in the normal range in the north of Iran.

\section{P011:18}

\section{OTOLOGIC ABNORMALITIES OF KLIPPEL-FEIL} SYNDROM: COMPUTED TOMOGRAPHY FINDINGS

\author{
A. Arslanoglu $^{1}$, N. Yildirim ${ }^{2}$ \\ ${ }^{1}$ Van Military Hospital, Van, Turkey; ${ }^{2}$ Van Yuzuncu Yil \\ University, Van, Turkey
}

Klippel-Feil Syndrom is a congenital vertebral or semivertebral fusion and/or atlas and axis deformity, that is expressed as short neck, low hair line and limited neck mobility.

Various systemic involments also accompany the syndrom including ear pathologies. Wide range of otologic abnormalities are seen in all three compartments of the ear (external, middle and inner ear) as well as internal acoustic canal and vestibular aquaduct.

We herewith present 20 cases, of which $12(\% 60)$ displayed most of the reported ear malformations including microtia, external ear canal stenoses, chronic ear malformations and their sequela, ossicular malformations, Mondini, and Michel deformity of inner ear, deformed internal acoustic canal and wide vestibular aquaduct, which are proved by Computed Tomography findings and audilogical and clinical manifestations.

\section{P011:19}

\section{RHINOLITHIASIS: CLINICAL, RADIOLOGICAL AND MINERALOGICAL FEATURES}

\author{
A. Arslanoglu $^{1}$, N. Yildirim ${ }^{2}$ \\ ${ }^{1}$ Van Military Hospital Department of Radiology, \\ Van, Turkey; ${ }^{2}$ Yuzuncuyil University Medical Faculty, Van, \\ Turkey
}

\section{OBJECTIVE}

The objective of the study is to review the clinical and radiological features of the rhinolithiasis and mineralogical-chemical composition of the rhinoliths with the aim of shedding light on the etiopathogenesis of this rare clinical entity.

\section{METHODS}

Our subjects are consisted of 8 cases of rhinolithiasis. Clinical presentations, signs and symptoms of the patients with radiological findings are herewith presented. X-ray diffraction analyses of the stones were also carried out to assess their mineralogical composition.

\section{RESULTS}

Nasal obstruction appeared to be the chief symptom in 6 of the patients and was present in all 8 , followed by nasal discharge, facial pain and epistaxis. Neither exogenous nor endogenous nidus was identified in any of the cases. Mineralogical analyses of the rhinoliths revealed Whitlockite $[(\mathrm{Ca}, \mathrm{Mg}) 3$ (PO4)2] in one and Dahllite $[\mathrm{Ca} 5(\mathrm{PO} 4, \mathrm{CO} 3) 3 \mathrm{OH}]$ in 7 cases.

\section{CONCLUSION}

We hypothesized that, some substances or objects of organic origin form the nidi of the rhinoliths and disintegrated over the course of mineral precipitation with the help of local inflammatory enzymes. This hypothesis was supported by radiological and microscopic findings. All rhinoliths were removed either under direct rhinoscopy or nasal endoscopy with success.

\section{PO11:20}

\section{HEMIFACIAL SPASM ORIGINATED BY A VERTEBRAL DOLICHOECTASIA}

M. Arantes Da Silva, R. Garcia, M. Costa, J. Resende Pereira

Hospital Pedro Hispano Matosinhos Portugal

\section{PURPOSE}

Hemifacial spasms (HFS) are characterized by chronic twitching or spasms of one side of the face. Most cases are associated with a small vascular loop compression. Rarely it's associated with a vertebral artery dolicoectasia. We report magnetic resonance imaging (MRI) and magnetic resonance angiography (MRA) findings of a right vertebra artery dolicoectasia compressing the facial nerve and causing HFS.

METHODS

Correlation of imaging and clinical findings. 


\section{RESULTS}

A 49 year old women, complained of an intermittent tight feeling in her right eye and in her upper cheek, exacerbated by eye movement initiated several months before admission. She had no medical problems, including no history of facial palsy. The neurological examination was normal, except for typical right HFS. High-resolution MRI and MRA of the brain showed indentation of the brainstem, particularly of the caudal pons, at the root entry zone of the right facial nerve by an enlarged and tortuous vertebral artery. She started treatment with periodic botulinum subcutaneous injections, with relief of the symptoms. CONCLUSIONS

HFS is an induced movement disorder characterized by unilateral, intermittent, tonic or clonic contractions of muscles innervated by the ipsilateral facial nerve. The most common etiology is a vascular loop compression at the root exit zone of the nerve. Antero-inferior cerebellar artery and postero-inferior cerebellar artery are generally the vessels involved. Vertebral dolichoectasia artery is a rare cause. This case demonstrates that MRI and MRA should be considered the initial screening procedures in the assessment of patients with HFS. The role of these neuroradiologic studies is not only to detect the vascular structures running adjacent to the root exit zone that might distort the facial nerve and compress the brain stem around the root exit zone, but also to exclude other causes like neoplasm or multiple sclerosis plaque.

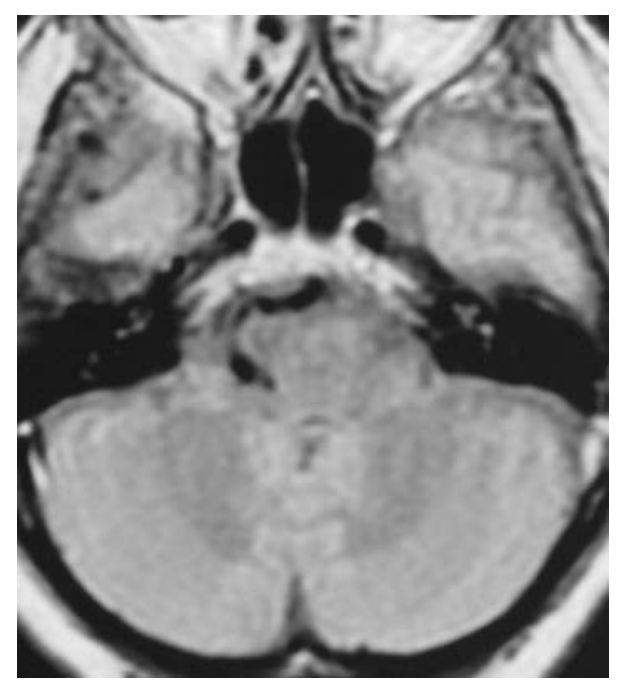

\section{P011:21}

\section{DEMONSTRATION OF BRACHIAL PLEXUS LESIONS WITH MRI}

\author{
A. Aralasmak, K. Karaali, C. Cevikol, \\ U. Senol \\ Akdeniz University, Department of Radiology, Antalya, Turkey
}

\section{PURPOSE}

In this presentation normal MRI anatomy and various lesions of the brachial plexus are shown.

\section{METHODS}

We reviewed the cases referred to our MR unit for brachial plexopathy within one year. For the MRI examination, spine coil was used with a slice thickness of 3 or $4 \mathrm{~mm}$. Smaller FOVs were used to visualize the exiting nerve roots. Sagittal views through the cervical spine were added to rule out degenerative findings or spinal cord lesions.

\section{RESULTS}

In neonatal patient group, there were findings of thickening and enhancement of the brachial plexus suggesting birth injury. Other associated findings were clavicula fracture, hematoma within the sternoceidomastoid muscle, pseudomeningocele, root avulsion or exiting root enhancement. In the young and middle age group, there were discopathy and radiculopathy, Chiari malformation with syrinx, posttraumatic neuroma and posttraumatic root avulsion with pseudomeningocele. In middle and older age group, there were metastases to brachial plexus from lung or breast cancer and radiation plexopathy following the neck cancer.

\section{CONCLUSION}

MRI is valuable in brachial plexus imaging. In neonates, traumatic injury was the most common encountered pathology while in middle and older ages, it was metastasis. In young-middle ages, degenerative changes and cord lesions are most likely. Examples of these pathologies are shown in our presentation.

\section{P011:22}

\section{ATYPICAL INTRACRANIAL INFECTIONS}

O. Algin, B. Hakyemez, M. Parlak

Uludag Unv. Faculty of Medicine, Bursa, Turkey

Atypical intracranial infections are relatively rare, but the consequences of a missed or delayed diagnosis are significant. CT and MR have become valuable imaging modalities in the evaluation and management of most intracerebral infections. Magnetic resonance imaging is the modality of choice for the characterization of intracranial inflammation, by defining ependyma, white matter, gray matter, and leptomeningeal involvement. Hemorrhage is well shown, but calcification is difficult to identify. Intravenous Gd-DTPA is useful in the evaluation of B-b barrier abnormalities. Although it is not often possible to make a specific microbiologic diagnosis, MR appearances are usually helpful in excluding other abnormalities, such as neoplasm or infarction. Knowledge of the number and size of individual lesions is invaluable in monitoring therapy. We report many cases of intracranial infections with atypical CTand MR findings, and attempt to correlate these findings with the pathophysiology. We also examine the salient findings reported for atypical intracranial infections. 


\section{PO11:23}

\section{IMAGING FINDINGS OF INTRACRANIAL INFECTIONS}

\section{O. Algin, B. Hakyemez, M. Parlak}

Uludag Unv. Faculty of Medicine. Radiology Department, Bursa, Turkey

The ability to detect and differentiate intracranial infection has markedly improved, first with the introduction of computed tomography and, more recently, with magnetic resonance. MR imaging is the procedure of choice in evaluating suspected intracranial infections because of its inherent contrast resolution, multiplanar capability, improved sensitivity in the posterior fossa, sensitivity to the presence of subacute, and chronic hemorrhage, and its sensitivity to the detection of meningeal disease on postcontrast images. The sensitivity of MR to foci of increased water content, seen in most parenchymal infections, the lack of bone artifact, and the multiplanar capability of MR have led to this preeminence. Computed tomography remains useful primarily in those infections associated with intracranial calcification, such as TORCH syndrome. Although it is not often possible to make a specific microbiologic diagnosis, CT and MR appearances are usually helpful in excluding other abnormalities, such as neoplasm or infarction. This exhibition summarizes the most recent $\mathrm{CT}$ and MR findings of intracranial infection.

\section{P011:24}

\section{THE VALUE OF COMPUTED TOMOGRAPHY PERFUSION EXAMINATION IN THE EVALUATION OF TISSUE INFILTRATION IN HEAD AND NECK CANCER}

A. Trojanowska, P. Orzechowski, P. Trojanowski, J. Klatka, A. Drop

University Medical School of Lublin, Lublin, Poland

\section{AIM}

We evaluated CT perfusion (CTP) images in patients with head and neck squamous cell carcinoma, in order to define infiltration of adjacent tissues.

METHODS AND MATERIAL

CTP was attempted in 12 patients with head and neck cancer (proven by biopsy) after conventional head and neck contrastenhanced computed tomography examination. Data were postprocessed by using deconvolution-based perfusion analysis, with the application of CT Perfusion 4 software (General Electric Medical Systems. Milwaukee, WI) on a GE Advantage Windows workstation.. Ipsilateral and contralateral common carotid artery was used as arterial input vessels. Postprocessing-generated maps showed mean transit time (MTT), blood volume (BV), blood flow (BF), and capillary permeability surface product (PS). Regions of interest were placed over the primary tumor site, salivary glands, muscles, base of tongue and subcutaneous fat. Average values of perfusion parameters were compared. Histologic specimens were obtained and the results of histological examination were compared with perfusion maps data. Sensitivity, specificity, positive and negative predictive value was calculated.

RESULTS

CTP was not possible in two patients. In the remaining 10 patients, average values of $\mathrm{BF}$ and $\mathrm{BV}$ were significantly higher in primary tumor than in tongue base or adjacent muscular structures $(\mathrm{P}<0.05)$ The MTT was significantly reduced in primary tumors compared to adjacent normal structures $(\mathrm{P}<0.05)$

CONCLUSION

CTP shows promise in defining local tumor spread and the invasion of adjacent structures. CT perfusion parameters may be applied to help in differentiation between head and neck squamous cell carcinoma and normal unaffected tissue.

\section{Spine and Spinal Cord Diseases}

\section{P012:1}

\section{DOUBLE READING OF SIPINAL MRI. 3-MONTH-RESULTS}

M. Serrallonga, E. Grivë, C. Schorlemmer, H. Billing

${ }^{1}$ Telemedicine Clinic, Barcelona, Spain

\section{PURPOSE}

To share our double reading (DR) experience. Telemedicine Clinic provides teleradiology service for public and private hospitals throughout Europe. Radiologists from several European countries report on exams. The aim of DR is to achieve the highest diagnostic accuracy through the professional expertise of two radiologists and to reduce the incidence of diagnostic errors. METHODS

DR has been performed in $100 \%$ of cases. We have retrospectively reviewed spinal DR done by six experienced Neuroradiologists from January 1st to March 31st 2007. All patients were adult outpatients. Following our guidelines, our grading system defines discrepancies as Grade 0 (full agreement), 1 (minor disagreement: changes with no clinical consequences), 2 (moderate disagreement: changes made unlikely to have significant clinical consequences), 3 (significant disagreement: changes with potential clinically significance) and 4 (different interpretation: high potential for clinically consequences).

For grade 0 , the report issued by the first reader was approved. For grade 1 , the report with the changes introduced by the second reader was considered as the final report. In case of grade 2 to 4 , the changes were communicated to the first reader for discussion and to give him/her the possibility to agree or disagree. When no consensus was achieved, the Section Leaders decided the final diagnosis.

\section{RESULTS}

Out of a total of 3024 reports (1077 cervical, 106 thoracic, 1643 lumbar, 89 whole spines) most disagreements were of no clinical consequences $(51.42 \%$ Grade $0,36.21 \%$ Grade 1 and $8.33 \%$ 
Grade 2). Clinically important disagreements were about $4 \%$ (3.80\% Grade 3 and $0.23 \%$ Grade 4$)$.

\section{CONCLUSIONS}

A systematic and controlled DR approach assures high quality reports. Diagnostic errors and slip mistakes are lowered, though most of the discrepancies were due to different reporting styles and structures. This highlights the importance of a unified reporting style for a better communication among radiologists and with the referring physicians.

\section{PO12:2}

\section{NORMAL THORACIC SPINAL CORD DIAMETER IN MRI}

U. Senol $^{1}$, G. Yarar ${ }^{1}$, C. Andic ${ }^{2}$, C. Cevikol ${ }^{1}$, K. Karaali ${ }^{1}$, E. Luleci ${ }^{1}$ ${ }^{1}$ Akdeniz University, Department of Radiology, Antalya, Turkey; ${ }^{2}$ Gaziantep University, Department of Radiology, Gaziantep, Turkey

\section{PURPOSE}

To obtain normal thoracic spinal cord diameter values in order to use in diagnosis and follow-up of the spinal cord atrophy with MRI.

\section{METHODS}

Eighty volunteers who do not have any neurological disease history and complaint were included in the study. Ten volunteers were then excluded because of the findings such as serious cervical disc herniation, minimal scoliosis, and suboptimal MRI examination. T1-weighted sagittal images of the thoracic spinal cord were obtained including Th 1-Th 12 levels, and AP diameter of the cord was measured at all vertebral levels. Axial images were also obtained at Th1, Th5 and Th9 levels and AP and transverse diameters were measured at these levels. The relation between AP diameters measured at the sagittal plan and age, height and, body weight of the cases were investigated.

\section{RESULTS}

At all levels, spinal cord AP diameters were ranged between 6.1$6.5 \mathrm{~mm}$. No significant correlation was found with age, height and body weight. In AP diameter of the cord, there was no statistically significant difference between sexes.

\section{CONCLUSION}

Spinal cord diameter shows no significant variation. To evaluate spinal cord atrophy with MRI, the measurements obtained in this study can be used, however, further comparative studies including the patients with spinal cord disease are necessary.

\section{PO12:3}

\section{SPINAL CORD TRACTOGRAPHY IN (PARTIAL) CONSUMATION OF CEREBROSPINALFLUID (CSF)}

\author{
A. Herneth $^{1}$, G. Kasprian ${ }^{2}$, D. Prayer ${ }^{3}$ \\ ${ }^{1}$ Department of Radiodiagnostics, Medical University Vienna, Vienna, \\ AUSTRIA; ${ }^{2}$ Departement of Neurology, Medical University Vienna, \\ Vienna, Austria; ${ }^{3}$ Deapartmetn of Neuroradiology, Mediacl Universi- \\ ty Vienna, Vienna, Austria
}

\section{PURPOSE}

Based on the assumption that diffusion-tensor (DT)sequences of parts of the spinal cord not surrounded by CSF, may be less vulnerable to pulsation artifacts than normal parts of the spinal cord, the utility of fibertracking in spinal pathologies with (partial) consumation of the surrounding CSF spaces was tested.

\section{PATIENTS AND METHODS}

In 2 patients (clinical questions: (1) relapse after several operations of a spinal astrocytoma in the thoracic cord, (2) bony cervical spinal-canal stenosis on degenerative basis), conventional MRimaging (sagittal T2-weigted, T1, weighted, STIR-images, axial T2-weighted images, contrast-enhanced sagittal T1-weighted images, diffusion-weighted images (patient1) were done on a $1.5 \mathrm{~T}$ superconducting unit. In both a customized DT sequence 24 directions, slice thickness $5 \mathrm{~mm}$, field of view $230 \mathrm{~mm}$, maximum b value of 1000) was applied.. Fibertracking was performed on a Philips workspace console.

RESULTS

In patient (1) the spinal cord could only be delineated on the images, generated by fibertracking, allowing the diagnosis of a secondary tethering of the spinal cord to the region of the postoperative scar (Fig 1). In patient (2) the clinical presentation could be clearly correlated to the site of fiber destruction on the tractography-images.

\section{CONCLUSION}

Tractography of the spinal cord in case of partial consumption of the surrounding CSF spaces seems to be feasible, and may add diagnostic information of prognostic and/or therapeutic relevance.

Fig 1: Tractography in pat 1 , showing the thethering of the spinal cord to the postoperative scar

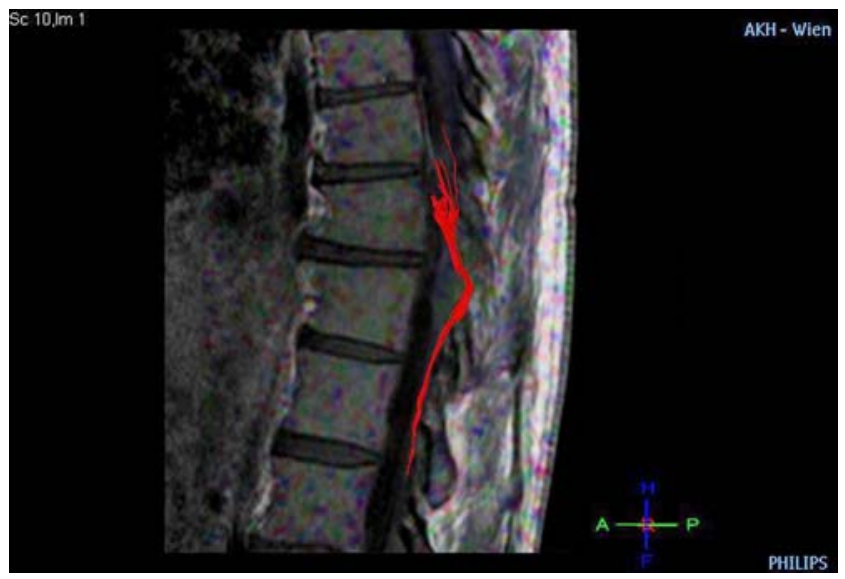

\section{P012:4}

FROIN'S SYNDROME: MAGNETIC RESONANCE IMAGING APPEARANCES

S. Mirza, W. Adams, R. Corkhill

${ }^{1}$ Department of Neuroradiology, Derriford Hospital,

Plymouth, United Kingdom 
Georges Froin described the condition of xanthochromic cerebrospinal fluid that rapidly clots on standing due to obstruction of the spinal subarachnoid space one hundred years ago in 1903. We report on three cases where this syndrome, caused by different pathologies, can be implied from magnetic resonance imaging.

This series comprises three patients all of whom had different pathologies that included ependymoma, prolapsed intervertebral disc and dermoid tumour, all of which caused obstruction of the spinal canal and development of Froin's syndrome. In all three cases, Magnetic Resonance imaging (MRI) was carried out. In all three cases signal return from the cerebrospinal fluid caudal to the obstructing pathology was higher than from that above the lesion especially on T2-weighted imaging. The possible patho-physiological mechanisms of this previously undescribed phenomenon, including increased levels of protein in association with other paramagnetic species as well as differential rates of cerebrospinal fluid flow in different parts of the cord are discussed.

Magnetic resonance imaging is now becoming the imaging technique of choice for evaluation of the spinal canal and it's contents and these cases show that Froin's syndrome can be a recognizable feature on MRI. We discuss the possible causes of this radiological phenomenon and postulate that this appearance on MRI may indicate a higher degree of obstruction.

\section{P012:5}

\section{SYMPTOMATIC VERTEBRAL HEMANGIOMA IN A PREGNANT PATIENT TREATED BY VERTEBROPLASTY AND SEGMENTAL FIXATION FOLLOWING ENDOVASCULAR TREATMENT}

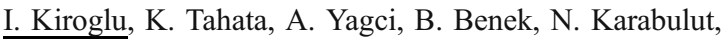 \\ B. Cirak \\ Pamukkale University School of Medicine, Denizli, Turkey
}

\section{PURPOSE}

Hemangiomas of the vertebral column are common and it remains silent in the vast majority of the subjects afflicted. Pregnancy is a known risk factor for symptomatic conversion of the previously silent vertebral hemangiomas. However, the occurrence is rare with only 26 cases reported in the English literature. In this paper, we report a case of vertebral hemangioma during pregnancy, presented with acute onset of upper back pain and progressive paraplegia.

\section{MATERIAL AND METHOD}

A previously healthy 22-year-old, gravid 2, para 1 woman, was admitted to our hospital due to acute onset of upper back pain and rapidly progressive paraplegia in her 36th week of pregnancy. She mentioned progressive lower extremity weakness and paraplegia progressed within a few days. Magnetic resonance imaging (MRI) studies showed a vertebral tumor occupying the entire T4 vertebral body, pedincules, transverse, spinous processes and right paravertebral soft tissue component with epidural extension encroaching on the spinal canal. Results Endovascular embolization of vascular vertebral afferences following caesarian section was performed and then, patient reported a dramatic improvement.
Two years after embolization, she has been readmitted with the complaint of severe upper back pain. MR and CT examinations revealed that spinal cord was compressed. Then, She underwent operation and vertebroplasty was performed through T4 pedicles bilaterally. The postoperative period was uneventful, the patient's complaints disappeared.

\section{CONCLUSION}

The neurological compromise of this disorder can be very devastating. Warning signs can be falsely interpreted as complaints related to pregnancy and can give rise to diagnostic delays. Therefore, this disease should also be investigated in the differential diagnosis of myelopathy in pregnant patients. Although high symptomatic recurrence rates and limited experiences in pregnant patients, endovascular embolization may be an alternative treatment choice for patients inwhom the surgery is contraindicated.

\section{P012:6}

\section{A CASE OF PRIMARY SOLITARY AMYLOIDOMA OF THE LUMBAR SPINE}

J. Kazaoka, I. Shirouzu, S. Amemiya, I. Matsuda, H. Akai, M. Noda, T. Machida

Kanto Medical Center NTT EC, Tokyo, Japan

\section{PURPOSE}

Primary solitary amyloidoma of the lumbar spine is extremely rare. We report a case of lumbar amyloidoma which caused neurological compromise due to cauda equina compression.

\section{CASE REPORT}

A 77-year-old man presented with lower back pain and weakness in the right leg after 7 months history of numbness radiating down the right leg. 3 months before, he underwent a magnetic resonance imaging (MRI) scan of lumbar spine at an outside hospital, and an extradural mass attached to the yellow ligament on the right side at L3-L4 was revealed. A MRI scan in our hospital demonstrated an enlargement of the mass, which had high signal intensity on T1weighted images and low signal intensity on T2-weighted images. No apparent enhancement was observed after intravenous administration of gadolinium. Dural sac was heavily compressed by the mass posterior to the right at L3-L4. The characteristics of the mass at MRI scan and its relatively acute enlargement resembled a hemorrhage into a cystic lesion. However, the mass removed with L3-L5 laminectomy was solid lesion sized $2.5 \times 1.8 \mathrm{~cm}$ in diameter. Histologically, the mass contained eosinophilic material, hyaline substance, and granulomatous tissue. Examination of a Congo red-stained section revealed amyloid deposition to the yellow ligament. Postoperative investigation did not show any evidence of a disease causing generalized amyloidosis. The weakness in the right leg was almost completely resolved after the surgery, and there was no evidence of any recurrence.

\section{CONCLUSION}

Primary solitary amyloidoma of the lumbar spine is an extremely rare compressive epidural lesion. The imaging findings of our case were consistent with those of previously reported cases. The 
course of our case suggested that amyloidoma may present relatively acute enlargement and worsening of neurological compromise.

\section{P012:7}

\section{OSTEOCHONDROMA OF C5 VERTEBRA PRESENTING AS COMPRESSIVE MYELOPATHY IN A PATIENT WITHOUT MULTIPLE EXOSTOSES}

\author{
$\underline{\text { V. Katsaros }}^{1}$, T. Flaskas ${ }^{2}$, E. Sourtzis ${ }^{2}$, S. Gatzonis ${ }^{2}$, \\ A. Savvani $^{3}$, S. Lionis ${ }^{1}$, D. Sakas ${ }^{2}$ \\ ${ }^{1}$ IKA Oncology Hospital, Department of CT and MRI, Athens, \\ Greece; ${ }^{2}$ Evangelismos Hospital, Department of Neurosurgery, \\ University of Athens, Athens, Greece; ${ }^{3}$ Evangelismos \\ Hospital, Department of Pathology, Athens, Greece
}

\section{STUDY DESIGN}

A case report.

\section{OBJECTIVE}

To highlight the importance of early diagnosis and treatment of vertebral exostosis.

SUMMARY OF BACKGROUND DATA

Osteochondroma, or exostosis, is the most common of all benign bone tumors. Vertebral osteochondromas contribute only 1.3$4.1 \%$ of all osteochondromas. Few cases of spinal cord compression caused by solitary cervical exostoses have been reported. MATERIALS AND METHODS

A solitary exostosis in the middle-right of the neural arch of the fifth cervical vertebra, causing compression of the spinal cord documented on both magnetic resonance and computed tomographic examinations, is reported in a 46-year-old woman who had pyramidal syndrome and deep sensation loss of both legs. RESULTS

Vertebral exostosis was suspected by magnetic resonance imaging and confirmed by histological examination. Surgical decompression was followed by complete resolution of the neurological impairments. CONCLUSION

Accurate preoperative diagnosis of vertebral exostoses is possible using magnetic resonance imaging and computed tomography. Early excision avoids the development of a permanent neurologic deficit.

\section{P012:8}

\section{SPECULATION ABOUT THE DISTINCTION BETWEEN THE FINDINGS OF SPINAL EDEMA AND CEREBRAL EDEMA ON MRI}

M. Hiratsuka, I. Anno, A. Kunimatsu, M. Minami Tsukuba University Hospital, Tsukuba, Japan

The MRI findings of spinal edema have an interesting point that the gray matter indicates higher intensity than the white matter by T2WI. For example, we found this point in case of the spinal sarcoidosis. But in many cases the cerebral edema appears as high intensity of white matter. The purpose of this exhibit is to review various diseases showing edematous changes and discuss about this point.

\section{THIS EXHIBIT CONTENTS}

1) Reviews of the spinal anatomy and pathophysiology of spinal edema 2) Reviews of various diseases which show spinal edematous changes on MRI especially by T2WI contain granulomatous, infectious, vascular, and other diseases 3) Reviews of the cases of cerebral edematous changes on MRI contain the case of radiation injury. 4) Distinctions between the spinal edema and the cerebral edema on MRI 5) Speculation about the distinctions based on various diseases which show edematous changes on MRI SUMMARY

1) In various diseases T2WI shows hyperintensity indicating edematous changes. In the spinal sarcoidosis MRI findings show an interesting point that the gray matter shows more hyperintensity than the white matter. 2) In the cerebrum the white matter shows more hyperintensity than the gray matter reflect the edema in maney cases. 3) This distinction suggests some speculations. We discuss about it based on various diseases and speculate about the machanism from their anatomical features and pathophysiology.

\section{P012:9}

\section{MODIFY IMAGING TECHNIQUES FOR EXAMINATION OF BAMBOO SPINE AT PRASAT NEUROLOGICAL INSTITUTE}

S. Chanyawattiwongse, K. Srisubat, C. Charoenwikrom, C. Saritcharoenkha

Prasat Neurological Institute, Bangkok, Thailand

\section{PURPOSE}

A 54 year old Thai male was a patient of Prasat Neurological Institute and was diagnosed of bamboo spine with fracture and dislocate of C6-7. Ankylosing spondylitis(AS) is a rare condition that can cause back and neck pain. It is a rheumatic inflammation disease characterized by ossification of the sacroiliac joints, spinal discs and ligaments. Eventually, the spine becomes fused across the disc spaces, resulting in the characteristic radiographic finding of 'bamboo spine'. The purposes of this report were to describe the imaging techniques for evaluating the bamboo spine.

\section{METHODS}

The diagnosis of AS is based on evaluating the patient's symptoms, a physical examination, blood tests genetic marker (HLA-B27 gene), CT scan and plain radiographs (x-ray) findings. Magnetic resonance imaging (MRI) are also helpful to demonstrate soft tissues better than plain radiographs, but the limitation of mobility of the patient is difficult to position when performing the imaging. In this case we performed the diagnosis with plain radiograph, CT scan and MRI of cervico-thoracic spine.

Plain radiograph: Cervico-thoracic x-ray examination was done by swimming technique.

CT scan: As supine position is not possible, we asked the patient to sit on CT scan table like riding on motorcycle and put the face on the head supporter. 
MRI: Flex coil is only available for imaging in this case. Contiguous $(3 \mathrm{~mm})$ sagittal, and transverse T1 weighted spin echo and $\mathrm{T} 2$ weighted gradient echo images were obtained.

\section{RESULTS}

Plain radiographs and $\mathrm{CT}$ scan show characteristic changes of vertebral bodies before surgery. MRI reveals evidence of spinal cord, intervertebral discs and subarachnoid space of cervicothoracic spine that can not detect on plain radiographs and CT scan. After surgery, plain radiographs demonstrate the plate, screws and wiring position, it also shows the alignment of the spine.

CONCLUSIONS

With modify imaging techniques, AS is more readily detectable and manageable.

\section{P012:10}

\section{ADHESIVE ARACHNOIDITIS: A SIMPLE TECHNICAL EXPEDIENT TO CONFIRM MRI DIAGNOSIS}

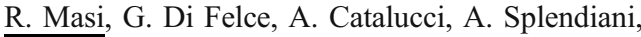

M. Gallucci

University of L'aquila, L'Aquila, Italy

To demonstrate the outcomes of lumbo-sacral adhesive arachnoiditis by means of diagnostic imaging techniques is not always easy. In addition, the clinical symptomatology poses problems of differential diagnosis with several diseases. MRI allows visualisation of the abnormal intracanalar course of the involved roots, but it does not helps to accomplish a diagnosis. The employment of contrast media adds important diagnostic information only in the acute phase or in particularly severe cases. A simple technical expedient, such as to perform an acquisition in the prone position, was helpful in our experience to demonstrate the lack of mobility of the involved roots. For the reliability and the reproducibility of the study in the prone position, we submitted 5 healthy volunteers to MRI examination in both prone and supine position demonstrating the mobility of the roots within the dural sac, and 10 patients with clinical suspicion of adhesive arachnoiditis. In the last group, the presence of root entrapment was confirmed in 7 cases; in the remaining three cases the radicular alteration was related to other diseases.

\section{P012:11}

\section{UPRIGHT MRI STUDY OF THE LUMBAR SPINE: PHYSIOLOGICAL CHANGES OF THE ANATOMICAL RELATIONS}

R. Masi, M. Di Fabio, A. Catalucci, A. Splendiani, M. Gallucci

University of L'aquila, L'aquila, Italy
The introduction of MRI units dedicated to the upright study of the rachis has allowed the neuroradiologist to better understand the status of the spine under physiological load. The changes of the spine that occur under loading and during motion have already been widely described by means of myelography. Aim of our study was to evaluate the possibility of dynamic MRI, to document the physiological changes of the anatomical structures of the lumbar spine and the relations with the canal size. Fifty healthy volunteers ( 25 males and 25 females, age ranging between 35 and 45 years) were submitted to MRI in both the upright and recumbent positions. Prior to the MRI examination, all subjects were submitted to neurological examination to rule out the presence of myelo-radicular diseases. The upright MRI examinations were randomly performed prior or subsequent to the study carried out in the recumbent position. The examinations were subsequently evaluated by two neuroradiologists who focused their attention on the measurements of lordosis, canal size, thickness of intersomatic discs and yellow ligaments. The possible alterations of signal intensity of the different structures were also described.

\section{P012:12}

\section{UPRIGHT MRI OF DISK HERNIATION: STATUS AND CHANGES OF ITS SIZE UNDER PHYSIOLOGICAL LOADING.}

M. Di Fabio, R. Masi, R. De Amicis, A. Catalucci, A. Splendiani, M. Gallucci

University of L'Aquila, L'Aquila, Italy

Though the clinical symptomatology of patients with lumbar disk herniation worsen under loading conditions, there is nowadays in the literature a lack of studies performed on large series describing the possible changes in disk herniation size depending on the upright position. The radicular compression may depend on many factors which are not necessarily related only to the disc itself. Namely, alterations of canal and foramina, ligamentous weakness, hydrodynamic changes of the dural sac can lead to pain in presence of a pathological disc. For this reason, we evaluated size changes of the disc herniation on 30 patients with disc disease, submitted to examinations on the upright and recumbent positions. We also evaluated the possible pre-existing causes and the signal intensity alterations of the vertebral end plates.

The results will be discussed.

\section{PO12:13}

ABSTRACT WITHDRAWN 